\title{
ON SUMS INVOLVING PRODUCTS AND QUOTIENTS OF L-FUNCTIONS OVER FUNCTION FIELDS
}

\author{
JEFFREY LiN THUNDER
}

\begin{abstract}
We estimate the sum of products or quotients of $L$-functions, where the sum is taken over all quadratic extensions of given genus over a fixed global function field. Our estimate for the sum of the quotient of two $L$-functions is analogous to a result of Schmidt where he estimates the sum of the quotient of two $L$-series, where the sum is over quadratic extensions of $\mathbb{Q}$ with absolute value of the discriminant less than a given bound.
\end{abstract}

\section{INTRODUCTION}

For well over a century zeta functions and $L$-series have played a prominent role in number theory and are objects of intense interest in their own right. We mention here just one example of how they occur. A basic notion in Diophantine geometry is the height, and counting points of given height or height less than a given bound on various varieties is a subject of much interest. Early on it was recognized that zeta functions appear in asymptotic estimates for the number of points of bounded height in projective spaces. A specific instance here is when one counts points of height no greater than a bound $B$ in $\mathbb{P}^{2}(\overline{\mathbb{Q}})$ that generate a quadratic extension of $\mathbb{Q}$. Schmidt in [9] gave an asymptotic estimate (in the bound $B$ ) for the number of such points, and showed that the coefficient of the main term in his asymptotic estimate involved a sum of quotients of $L$-series of the form

$$
\sum_{\substack{[F: \mathbb{Q}]=2 \\|\operatorname{Disc}(F)| \leq m}} \frac{L_{F}(1)}{L_{F}(3)},
$$

where $\operatorname{Disc}(F)$ is the discriminant of $F$ over $\mathbb{Q}$. In the appendix of [9] he proved asymptotic (in the parameter $m$ ) estimates for sums of the form

$$
\sum_{\substack{[F: \mathbb{Q}]=2 \\|\operatorname{Disc}(F)| \leq m}} \frac{L_{F}(s)}{L_{F}(t)}
$$


with certain restrictions on $s, t \in \mathbb{C}$, of course. As Schmidt remarks, sums like the one above have received interest before, going back all the way to Gauss, in fact.

Now for quite some time number theorists have realized that it is fruitful to also study function fields; says Weil in the foreword to [13] “... it goes without saying that the function fields over finite fields must be granted a fully simultaneous treatment with number fields ..." In particular, one can study heights over any global function field and hopefully gain insight into number fields in the process. It is in this spirit that the author together with Widmer in [12] derive asymptotic (in the height) estimates for the number of points of given height that generate an extension of given degree over a fixed function field, though not in all situations where we believe such estimates should hold. Together with Kettlestrings, in [7] we derive such estimates in the remaining previously unproven cases when one counts points that generate a quadratic extension of a fixed function field. This is analogous to Schmidt's work cited above, and unsurprisingly requires estimates for sums of quotients of $L$-functions over function fields analogous to the sums above considered by Schmidt.

Here we will develop machinery (specifically, Propositions 5-7 in $\S 4$ below) that allows one to estimate certain sums of products and quotients of $L$-functions. Loosely speaking, our methods follow those of Siegel in [10]; they are purposely less "geometric" and more "number-theoretic" in nature. As an example of the use of Propositions 5-7, we will prove estimates for the sum over the quotient and product of two $L$-functions. This includes the case of interest above in [7] that arises in the counting of points of given height generating a quadratic extension. Before stating these estimates, we first set some notation.

For a prime $p$ let $\mathbb{F}_{p}$ denote the finite field with $p$ elements and let $X$ be transcendental over $\mathbb{F}_{p}$, so that $\mathbb{F}_{p}(X)$ is a field of rational functions. Fix algebraic closures $\overline{\mathbb{F}_{p}}$ of $\mathbb{F}_{p}$ and $\overline{\mathbb{F}_{p}(X)} \supset \overline{\mathbb{F}_{p}}$ of $\mathbb{F}_{p}(X)$. In what follows, by global function field (or simply function field) we mean a finite algebraic extension $K \supseteq \mathbb{F}_{p}(X)$ contained in $\overline{\mathbb{F}_{p}(X)}$. For such a field $K$ we have $K \cap \overline{\mathbb{F}_{p}}=\mathbb{F}_{q_{K}}$ for some finite field $\mathbb{F}_{q_{K}}$ with $q_{K}$ elements; this field is called the field of constants of $K$. We write $g_{K}$ for the genus of $K$ and $\zeta_{K}$ for the zeta function of $K$ (defined explicitly below). The $L$-function $L_{K}$ is given by

$$
L_{K}\left(q_{K}^{-s}\right)=\left(1-q_{K}^{-s}\right)\left(1-q_{K}^{1-s}\right) \zeta_{K}(s)=\frac{\zeta_{K}(s)}{\zeta_{\mathbb{F}_{q_{K}}(X)}(s)} .
$$

It is well-known that $L_{K}$ is a polynomial of degree $2 g_{K}$ in $q_{K}^{-s}$ and all its zeros have $\Re(s)=1 / 2$ 
(see [11, Chap. V], for example). We denote the set of places of $K$ by $M(K)$.

Theorem 1. Suppose $K$ is a global function field with field of constants $\mathbb{F}_{q}, m$ is a positive integer and $\epsilon>0$. Suppose $s, t \in \mathbb{C}$ satisfy i) $\Re(s), \Re(t)>3 / 4+\epsilon$ and $\Re(s)+\Re(t)>2+2 \epsilon$ if $q$ is odd; ii) $\Re(s), \Re(t)>1 / 2+\epsilon$ and $\Re(s)+\Re(t)>3 / 2+2 \epsilon$ if $q$ is even. Then

$$
\begin{aligned}
& \sum_{\substack{[F: K]=2 \\
g_{F}=m, q_{F}=q}} L_{F}\left(q^{-s}\right) L_{F}\left(q^{-t}\right)=\frac{2 J_{K} q^{3-5 g_{K}} \zeta_{K}(2 s) \zeta_{K}(2 t) L_{K}\left(q^{-s}\right) L_{K}\left(q^{-t}\right)}{q-1} \sigma_{1}(s, t) q^{2 m} \\
& + \begin{cases}O\left(q^{m}\left(1+q^{2 m(5 / 4+\epsilon-\Re(s))}\right)\left(1+q^{2 m(5 / 4+\epsilon-\Re(t))}\right)\right) & \text { if } q \text { is odd, } \\
O\left(q^{m}\left(1+q^{2 m(1+\epsilon-\Re(s))}\right)\left(1+q^{2 m(1+\epsilon-\Re(t))}\right)\right) & \text { if } q \text { is even, }\end{cases}
\end{aligned}
$$

where

$$
\begin{aligned}
\sigma_{1}(s, t)= & \prod_{v \in M(K)}\left(1-q^{-2 \operatorname{deg}(v)}-\left(q^{-\operatorname{deg}(v)}-q^{-2 \operatorname{deg}(v)}\right)\left(q^{-2 s \operatorname{deg}(v)}-q^{-2(s+t) \operatorname{deg}(v)}+q^{-2 t \operatorname{deg}(v)}\right)\right. \\
& \left.+\left(1-q^{-\operatorname{deg}(v)}\right) q^{-(s+t) \operatorname{deg}(v)}\right)
\end{aligned}
$$

and the implicit constants depend only on $K$ and $\epsilon$.

Theorem 2. Suppose $K$ is a global function field with field of constants $\mathbb{F}_{q}, m$ is a positive integer and $\epsilon>0$. Suppose $s, t \in \mathbb{C}$ satisfy i) $\Re(s)>3 / 4+\epsilon, \Re(t)>1+\epsilon$ and $\Re(s)+\Re(t)>2+2 \epsilon$ if $q$ is odd; ii) $\Re(s)>1 / 2+\epsilon$ and $\Re(t)>1+\epsilon$ if $q$ is even. Then

$$
\begin{aligned}
& \sum_{\substack{[F: K]=2 \\
g_{F} \stackrel{=}{=}, q_{F}=q}} \frac{L_{F}\left(q^{-s}\right)}{L_{F}\left(q^{-t}\right)}=\frac{2 J_{K} q^{3-5 g_{K}} \zeta_{K}(2 s) L_{K}\left(q^{-s}\right)}{L_{K}\left(q^{-t}\right)(q-1)} \sigma_{2}(s, t) q^{2 m} \\
& + \begin{cases}O\left(q^{m}\left(1+q^{2 m(5 / 4+\epsilon-\Re(s))}+q^{2 m(5 / 2+2 \epsilon-2 \Re(t))}+q^{2 m(5 / 2+2 \epsilon-\Re(s)-\Re(t))}\right)\right. & \text { if } q \text { is odd, } \\
O\left(q^{m}\left(1+q^{2 m(1+\epsilon-\Re(s))}\right)\right) & \text { if } q \text { is even, }\end{cases}
\end{aligned}
$$

where

$\sigma_{2}(s, t)=\prod_{v \in M(K)}\left(1-q^{-2 \operatorname{deg}(v)}+q^{-2(s+1) \operatorname{deg}(v)}-q^{-(2 s+1) \operatorname{deg}(v)}-q^{-(t+s) \operatorname{deg}(v)}+q^{-(t+s+1) \operatorname{deg}(v)}\right)$ and the implicit constants depend only on $K$ and $\epsilon$.

Theorem 3. Suppose $K$ is a global function field with field of constants $\mathbb{F}_{q}, m$ is a positive integer and $\epsilon>0$. Suppose $s, t \in \mathbb{C}$ satisfy $\Re(s), \Re(t)>1+\epsilon$. Then

$$
\begin{aligned}
& \sum_{\substack{[F: K]=2 \\
g_{F}=m, q_{F}=q}} \frac{1}{L_{F}\left(q^{-s}\right) L_{F}\left(q^{-t}\right)}=\frac{2 J_{K} q^{3-5 g_{K}}}{L_{K}\left(q^{-s}\right) L_{K}\left(q^{-t}\right)(q-1)} \sigma_{3}(s, t) q^{2 m} \\
& + \begin{cases}O\left(q^{m}\left(1+q^{2 m(5 / 2+2 \epsilon-2 \Re(s))}+q^{2 m(5 / 2+2 \epsilon-2 \Re(t))}\right)\right) & \text { if } q \text { is odd, } \\
O\left(q^{m}\right) & \text { if } q \text { is even, }\end{cases}
\end{aligned}
$$


where

$$
\sigma_{3}(s, t)=\prod_{v \in M(K)}\left(1-q^{-2 \operatorname{deg}(v)}+q^{-(s+t) \operatorname{deg}(v)}-q^{-(s+t+1) \operatorname{deg}(v)}\right)
$$

and the implicit constants depend only on $K$ and $\epsilon$.

One can view the case where $K$ is a field of rational functions (at least for odd characteristic) in Theorem 2 as the analog of Schmidt's result over $\mathbb{Q}$ mentioned above. We note that letting $t=\Re(t) \rightarrow \infty$ in either Theorem 1 or 2 gives the sum of a single $L$-function. This can be done more directly using our methods, though the resulting estimate (i.e., error term) is the same. Letting $s=\Re(s) \rightarrow \infty$ in either Theorem 2 or Theorem 3 gives the sum of the reciprocal of a single $L$-function.

Corollary 1. Suppose $K$ is a global function field with field of constants $\mathbb{F}_{q}, m$ is a positive integer and $\epsilon>0$. Then for all $s \in \mathbb{C}$ with $\Re(s)>1 / 2+\epsilon$ if $q$ is even or $\Re(s)>3 / 4+\epsilon$ if $q$ is odd, we have

$$
\begin{aligned}
\sum_{\substack{[F: K]=2 \\
g_{F}=m, q_{F}=q}} L_{F}\left(q^{-s}\right)=q^{2 m} & \frac{2 J_{K} q^{3-5 g_{K}} \zeta_{K}(2 s) L_{K}\left(q^{-s}\right)}{q-1} \\
& \times \prod_{v \in M(K)}\left(1-q^{-2 \operatorname{deg}(v)}+q^{-2(s+1) \operatorname{deg}(v)}-q^{-(2 s+1) \operatorname{deg}(v)}\right) \\
& + \begin{cases}O\left(q^{m}\left(1+q^{2 m(5 / 4+\epsilon-\Re(s))}\right)\right) & \text { if } q \text { is odd, } \\
O\left(q^{m}\left(1+q^{2 m(1+\epsilon-\Re(s))}\right)\right) & \text { if } q \text { is even, }\end{cases}
\end{aligned}
$$

where the implicit constants depend only on $K$ and $\epsilon$.

Corollary 2. Suppose $K$ is a global function field with field of constants $\mathbb{F}_{q}, m$ is a positive integer and $\epsilon>0$. Then for all $t \in \mathbb{C}$ with $\Re(t)>1+\epsilon$ we have

$$
\begin{aligned}
& \sum_{\substack{[F: K]=2 \\
g_{F}=m, q_{F}=q}} \frac{1}{L_{F}\left(q^{-t}\right)}=q^{2 m} \frac{2 J_{K} q^{3-5 g_{K}}}{L_{K}\left(q^{-t}\right) \zeta_{K}(2)(q-1)} \\
& \\
& + \begin{cases}O\left(q^{m} q^{2 m(5 / 2+2 \epsilon-2 \Re(t))}\right) & \text { if } q \text { is odd, } \\
O\left(q^{m}\right) & \text { if } q \text { is even, }\end{cases}
\end{aligned}
$$

where the implicit constants depend only on $K$ and $\epsilon$.

Results of a similar nature to our Corollary 1, though only in odd characteristic, have been given by Hoffstein and Rosen [5] (when $K=\mathbb{F}_{q}(X)$ only) and Fisher and Friedberg [3], with later 
refinements by Chinta, Friedberg and Hoffstein in [1]. These works use metaplectic Eisenstein series and double Dirichlet series. In contrast, our methods here are rather pedestrian, using only "classical" machinery such as Dedekind's different theorem and Hurwitz's genus formula, though to be fair we also avail ourselves of results such as the Riemann hypothesis for curves over a finite field which are obviously still unproven in the number field case. Sums analogous to those in Corollary 1 but with $\mathbb{Q}$ in place of the function field were studied by Goldfeld and Hoffstein [4].

We remark that one could use these theorems to estimate the number of quadratic extensions $F$ of $K$ with given genus. Such an estimate is actually used in our proofs, in fact, and is another consequence of our analysis (specifically, Proposition 7). Such estimates are either not extant, or at least difficult to locate in the present literature, and improve on the quadratic case of the more general estimates obtained in $[2]$ (over $\mathbb{F}_{q}(X)$ and only in odd characteristic) and [6]. We also note that another ingredient of our proofs is a function field analog of the Polya-Vinogradov inequality (Theorem 4 in $\S 3$ ). This is likely of independent interest as well; we were unable to locate such an analog in the literature.

We end this introduction with some more notation to be used throughout the remainder and give an elementary result on the prime divisors occurring in a given effective divisor, a result that is analogous to well-known estimates in elementary number theory. Section 1 is a short discussion on $L$-functions, Euler products and how to use Möbius inversion to express the inverse of the Artin $L$-function. With this in hand, we can indicate explicitly the type of sums that must be estimated, which quantities should dominate, and which should only contribute to "error terms." Section 2 is devoted to a detailed study of the differents and discriminants that arise from quadratic extensions and the quadratic extensions that have a particular discriminant. This is rather routine in odd characteristic, but decidedly less so in characteristic two. In section 3 we reinterpret our $L$-functions in terms of generators and characters. We then study multiplicative characters in this context and prove our analog of the Polya-Vinogradov inequality. Section 4 is the heart of our work. Here we state and prove our main estimates: Propositions 5, 6 and 7. Using these we prove Theorems 1, 2 and 3 in the final section.

We will follow the usual conventions that empty sums are interpreted to be zero and empty products are interpreted to be one. 
For a function field $K$ we denote the divisor group, i.e., the free abelian group on the set of places $M(K)$, by $\operatorname{Div}(K)$. The degree map on $\operatorname{Div}(K)$, normalized to have image $\mathbb{Z}$, will be denoted $\operatorname{deg}_{K}$ or simply deg if the field is understood. We will always use capital script German letters $\mathfrak{A}, \mathfrak{B}, \ldots$ to denote divisors, with the sole exception of the zero divisor 0 . The support of a divisor $\mathfrak{A}$, that is, the (possibly empty) set of places $v$ for which $\operatorname{ord}_{v}(\mathfrak{A}) \neq 0$, will be denoted by $\operatorname{Supp}(\mathfrak{A})$. We say an effective divisor $\mathfrak{A}$ (that is, a divisor $\mathfrak{A} \geq 0$ ) is square-free if $\operatorname{ord}_{v}(\mathfrak{A})=1$ for all $v \in \operatorname{Supp}(\mathfrak{A})$. When we work in the case of characteristic 2 it will prove useful to separate out the square-free part of an effective divisor. Towards that end, for any divisor $\mathfrak{A} \geq 0$ we set

$$
\mathfrak{A}_{1}=\sum_{v \in \operatorname{Supp}(\mathfrak{A})} v, \quad \mathfrak{A}_{2}=\mathfrak{A}-\mathfrak{A}_{2}
$$

When two effective divisors $\mathfrak{A}$ and $\mathfrak{B}$ have disjoint support, we write $(\mathfrak{A}, \mathfrak{B})=0$.

Each place $v \in M(K)$ has a corresponding order function $\operatorname{ord}_{v}: K \rightarrow \mathbb{Z} \cup\{\infty\}$, whence an (ultrametric) absolute value; these absolute values lead one to the adele ring $K_{\mathbb{A}}$ (see [13] for all of the necessary background here). For any divisor $\mathfrak{A} \in \operatorname{Div}(K)$ we have the Riemann-Roch space

$$
L(\mathfrak{A})=\left\{\alpha \in K: \operatorname{ord}_{v}(\alpha) \geq-\operatorname{ord}_{v}(\mathfrak{A}) \text { for all } v \in M(K)\right\}
$$

which is a vector space of finite dimension $l(\mathfrak{A})$ over $\mathbb{F}_{q_{K}}$. The Riemann-Roch Theorem states that

$$
l(\mathfrak{A})=\operatorname{deg}_{K}(\mathfrak{A})+1-g_{K}+l\left(\mathfrak{W}_{K}-\mathfrak{A}\right),
$$

where $\mathfrak{W}_{K} \in \operatorname{Div}(K)$ is any divisor in the canonical class (see either [13, Chapter VI] or [11, Chapter I]). In particular, if $\operatorname{deg}_{K}(\mathfrak{A}) \geq 2 g_{K}-1$, then $l(\mathfrak{A})=\operatorname{deg}_{K}(\mathfrak{A})+1-g_{K}$, so that the number of effective divisors $\mathfrak{A} \in \operatorname{Div}(K)$ with fixed degree $\operatorname{deg}(\mathfrak{A})=m$ is bounded by the product of $q_{K}^{m}$ and a function depending only on $K$ :

$$
\sum_{\substack{\mathfrak{A} \in \operatorname{Div}(K) \\ \mathfrak{A} \geq 0 \\ \operatorname{deg}(\mathfrak{A})=m}} 1 \ll q_{K}^{m}
$$

for all non-negative integers $m$, where the implicit constant depends only on $K$. In addition to the Riemann-Roch spaces, we will often consider the subsets

$$
L^{\prime}(\mathfrak{A})=\left\{\alpha \in L(\mathfrak{A}): \operatorname{ord}_{v}(\alpha)=-\operatorname{ord}_{v}(\mathfrak{A}) \text { for all } v \in \operatorname{Supp}(\mathfrak{A})\right\}
$$


The zeta function is given by

$$
\zeta_{K}(s)=\sum_{\substack{\mathfrak{A} \in \operatorname{Div}(K) \\ \mathfrak{A} \geq 0}} q_{K}^{-s \operatorname{deg}_{K}(\mathfrak{A})}
$$

for $\Re(s)>1$, where the sum converges absolutely by $(0)$. The resulting function can be analytically continued to a function with simple poles at $s=0,1$ and precisely $2 g_{K}$ zeros, all with real part equal to $1 / 2$. See $\left[11\right.$, Chap. V], for example. The case where $K=\mathbb{F}_{q}(X)$, a field of rational functions, is particularly simple:

$$
\zeta_{\mathbb{F}_{q}(X)}(s)=\frac{1}{\left(1-q^{-s}\right)\left(1-q^{1-s}\right)}
$$

We will often use "Möbius inversion" on certain sums over divisors. The Möbius function on effective divisors $\mathfrak{A} \in \operatorname{Div}(K)$ is defined exactly as in the classical case: $\mu(\mathfrak{A})=0$ unless $\mathfrak{A}$ is square-free, in which case $\mu(\mathfrak{A})=1$ if $\# \operatorname{Supp}(\mathfrak{A})$ is even and $\mu(\mathfrak{A})=-1$ if $\# \operatorname{Supp}(\mathfrak{A})$ is odd. The inversion arguments all rely on the formula (see [12, Lemma 1])

$$
\sum_{0 \leq \mathfrak{C} \leq \mathfrak{A}} \mu(\mathfrak{C})= \begin{cases}1 & \text { if } \mathfrak{A}=0 \\ 0 & \text { otherwise }\end{cases}
$$

In addition to the Möbius function on effective divisors, we will also use the phi function:

$$
\phi(\mathfrak{A})=\prod_{v \in \operatorname{Supp}(\mathfrak{A})}\left(q_{K}^{\operatorname{ord}_{v}(\mathfrak{A})}-q_{K}^{\operatorname{ord}_{v}(\mathfrak{A})-1}\right)
$$

Lemma 0. Let $K$ be a function field. For all effective divisors $\mathfrak{A} \in \operatorname{Div}(K)$ and all $\epsilon>0$ we have

$$
\# \operatorname{Supp}(\mathfrak{A}) \leq \epsilon \operatorname{deg}(\mathfrak{A})+c(\epsilon)
$$

for some constant $c(\epsilon) \geq 0$ depending only on $K$ and $\epsilon$, and

$$
\sum_{0 \leq \mathfrak{B} \leq \mathfrak{A}} 1 \ll q_{K}^{\epsilon \operatorname{deg}(\mathfrak{A})}
$$

where the implicit constant depends only on $K$ and $\epsilon$.

Proof. For the first inequality, let $n$ be the least positive integer greater than $1 / \epsilon$. The number $d(n)$ of places of degree less than $n$ certainly depends only on $K$ and $n$ (whence $\epsilon$ ). Now if $\# \operatorname{Supp}(\mathfrak{A}) \geq d(n)$, then

$$
\operatorname{deg}(\mathfrak{A}) \geq n \# \operatorname{Supp}(\mathfrak{A})-n d(n),
$$


so that

$$
\epsilon \operatorname{deg}(\mathfrak{A})+d(n) \geq \# \operatorname{Supp}(\mathfrak{A})
$$

Since the maximum of $\# \operatorname{Supp}(\mathfrak{A})-\epsilon \operatorname{deg}(\mathfrak{A})$ over all effective divisors $\mathfrak{A}$ with \# $\operatorname{Supp}(\mathfrak{A})<d(n)$ depends only on $K$ and $n$ (whence $\epsilon$ ), the first inequality in Lemma 0 follows.

For the second inequality, we first note that the function

$$
\theta(\mathfrak{A})=\sum_{0 \leq \mathfrak{B} \leq \mathfrak{A}} 1
$$

satisfies $\theta(\mathfrak{A}+\mathfrak{B})=\theta(\mathfrak{A}) \theta(\mathfrak{B})$ whenever $(\mathfrak{A}, \mathfrak{B})=0$. Also, $\theta(n v)=n+1$ for all non-negative integers $n$ and places $v$. Now set $c_{1}=\max _{x \geq 0} \log _{q_{K}}(x+1)-\epsilon x / 2$ and $c_{2}=\max \left\{c_{1}, 0\right\}$. Clearly $c_{2}$ depends only on $q_{K}$ and $\epsilon$, so by the first part of the lemma we see that

$$
\sum_{v \in \operatorname{Supp}(\mathfrak{A})} c_{2} \leq(\epsilon / 2) \operatorname{deg}(\mathfrak{A})+c
$$

for all effective divisors $\mathfrak{A}$ and some $c \geq 0$ depending only on $K$ and $\epsilon$. Thus, for any effective divisor $\mathfrak{A}$

$$
\begin{aligned}
\log _{q_{K}}(\theta(\mathfrak{A})) & =\sum_{v \in \operatorname{Supp}(\mathfrak{A})} \log _{q_{K}}\left(\operatorname{ord}_{v}(\mathfrak{A})+1\right) \\
& \leq \sum_{v \in \operatorname{Supp}(\mathfrak{A})} c_{2}+\epsilon \operatorname{ord}_{v}(\mathfrak{A}) / 2 \\
& \leq(\epsilon / 2) \operatorname{deg}(\mathfrak{A})+c+(\epsilon / 2) \operatorname{deg}(\mathfrak{A}) \\
& =\epsilon \operatorname{deg}(\mathfrak{A})+c .
\end{aligned}
$$

\section{L-Functions And Euler Products}

Throughout this section we fix a function field $K$ and we set $q=q_{K}$. We start with the Euler product representation of the zeta function for $K$ :

$$
\zeta_{K}(s)=\prod_{v \in M(K)}\left(1-q^{-s \operatorname{deg}_{K}(v)}\right)^{-1}
$$

Suppose now that $F$ is a quadratic extension of $K$ with the same field of constants. We then may 
write

$$
\begin{aligned}
\zeta_{F}(s) & =\prod_{w \in M(F)}\left(1-q^{-s \operatorname{deg}_{F}(w)}\right)^{-1} \\
& =\prod_{v \in M(K)} \prod_{\substack{w \in M(F) \\
w \mid v}}\left(1-q^{-s \operatorname{deg}_{F}(w)}\right)^{-1} \\
& =\prod_{v \in M(K)} \prod_{\substack{w \in M(F) \\
w \mid v}}\left(1-q^{-s f(w) \operatorname{deg}_{K}(v)}\right)^{-1},
\end{aligned}
$$

where $f(w)$ denotes the residue class degree, as usual. We'll denote the ramification index by $e(w)$. Since we have a quadratic extension, we have only three possibilities to consider: there is only one place $w \mid v$ and it satisfies $e(w)=2, f(w)=1$; there is only one place $w \mid v$ and it satisfies $e(w)=1, f(w)=2$; there are two places of $F$ lying above $v$, both with ramification index and residue class degree equal to 1 . In the first case here we set $\chi(F / v)=0$, in the second we set $\chi(F / v)=-1$, and in the third case $\chi(F / v)=1$. Thus for any place $v \in M(K)$ we have

$$
\prod_{\substack{w \in M(F) \\ w \mid v}}\left(1-q^{-s \operatorname{deg}_{F}(w)}\right)^{-1}=\left(1-q^{-s \operatorname{deg}_{K}(v)}\right)^{-1}\left(1-\chi(F / v) q^{-s \operatorname{deg}_{K}(v)}\right)^{-1} .
$$

Now extend $\chi$ to all effective divisors $\mathfrak{A} \in \operatorname{Div}(K)$ by setting

$$
\chi(F / \mathfrak{A})=\prod_{v \in \operatorname{Supp}(\mathfrak{A})}(\chi(F / v))^{\operatorname{ord}_{v}(\mathfrak{A})} .
$$

Then

$$
\begin{aligned}
\zeta_{F}(s) & =\zeta_{K}(s) \prod_{v \in M(K)}\left(1-\chi(F / v) q^{-s \operatorname{deg}_{K}(v)}\right)^{-1} \\
& =\zeta_{K}(s) \sum_{\substack{\mathfrak{A} \in \operatorname{Div}(K) \\
\mathfrak{A} \geq 0}} \chi(F / \mathfrak{A}) q^{-s \operatorname{deg}_{K}(\mathfrak{A})},
\end{aligned}
$$

whence

$$
L_{F}\left(q^{-s}\right)=L_{K}\left(q^{-s}\right) \sum_{\substack{\mathfrak{A} \in \operatorname{Div}(K) \\ \mathfrak{A} \geq 0}} \chi(F / \mathfrak{A}) q^{-s \operatorname{deg}_{K}(\mathfrak{A})} .
$$

In view of this, we have the following.

Definition. Suppose $K$ is a function field with field of constants $\mathbb{F}_{q}$ and $F$ is a quadratic extension of $F$ with the same field of constants. For all $s \in \mathbb{C}$, set

$$
L_{F}^{*}\left(q^{-s}\right)=\frac{L_{F}\left(q^{-s}\right)}{L_{K}\left(q^{-s}\right)}=\prod_{v \in M(K)}\left(1-\chi(F / v) q^{-s \operatorname{deg}_{K}(v)}\right)^{-1}=\sum_{\substack{\mathfrak{A} \in \operatorname{Div}(K) \\ \mathfrak{A} \geq 0}} \chi(F / \mathfrak{A}) q^{-s \operatorname{deg}_{K}(\mathfrak{A})} .
$$


These $L_{F}^{*}$ are examples of Artin $L$-series, made particularly simple here since we are only considering quadratic extensions. Though the definition above is a formal one that doesn't address convergence issues, in fact $L_{F}^{*}$ is a polynomial of degree $2 g_{F}-2 g_{K}$ in $q^{-s}$. We express the reciprocal of $L_{F}^{*}$ with the help of the Möbius function.

Lemma 1. Let $K$ be a function field with field of constants $\mathbb{F}_{q}$ and suppose $F \supset K$ is a quadratic extension with the same field of constants. For all $s \in \mathbb{C}$ we have

$$
\left(L_{F}^{*}\left(q^{-s}\right)\right)^{-1}=\sum_{\substack{\mathfrak{B} \in \operatorname{Div}(K) \\ \mathfrak{B} \geq 0}} \mu(\mathfrak{B}) \chi(F / \mathfrak{B}) q^{-s \operatorname{deg}_{K}(\mathfrak{B})}
$$

Proof. Using (1) we have (with $\mathfrak{C}=\mathfrak{A}+\mathfrak{B}$ )

$$
\begin{aligned}
L_{F}^{*}\left(q^{-s}\right) \sum_{\mathfrak{B} \geq 0} \mu(\mathfrak{B}) \chi(F / \mathfrak{B}) q^{-s \operatorname{deg}_{K}(\mathfrak{B})} & =\sum_{\mathfrak{A} \geq 0} \chi(F / \mathfrak{A}) q^{-s \operatorname{deg}_{K}(\mathfrak{A})} \sum_{\mathfrak{B} \geq 0} \mu(\mathfrak{B}) \chi(F / \mathfrak{B}) q^{-s \operatorname{deg}_{K}(\mathfrak{B})} \\
& =\sum_{\mathfrak{C} \geq 0} \chi(F / \mathfrak{C}) q^{-s \operatorname{deg}_{K}(\mathfrak{C})} \sum_{0 \leq \mathfrak{B} \leq \mathfrak{C}} \mu(\mathfrak{B}) \\
& =1 .
\end{aligned}
$$

Given all the above, we see that to estimate a sum of products or quotients of $L$-functions, we must estimate sums of the form

$$
\sum_{\substack{[F: K]=2 \\ g_{F}=m, q_{F}=q}} \chi(F / \mathfrak{C})
$$

for effective divisors $\mathfrak{C} \in \operatorname{Div}(K)$. Note that $\chi(F / \mathfrak{C})=1$ when $\mathfrak{C} \in 2 \operatorname{Div}(K)$, except when some place in the support of $\mathfrak{C}$ ramifies in $F$. Heuristically, one expects sums of the form (2) where $\mathfrak{C} \notin 2 \operatorname{Div}(K)$ to be "small" in some sense, so that the main contribution to our sums of $L$-functions should come from those $\mathfrak{C}$ where $\mathfrak{C} \in 2 \operatorname{Div}(K)$. Our goal is therefore two-fold: evaluate these sums when $\mathfrak{C} \in 2 \operatorname{Div}(K)$ and show the rest only contribute an error term of smaller magnitude. This is precisely what we achieve in Propositions 5-7 below.

\section{The Different and Discriminant}

As in the case of number fields, we turn to the discriminant as a means of conveniently indexing the quadratic extensions of a given function field $K$. We first dispense with the inseparable case, however. Suppose that $[F: K]=2$ and $q_{F}=q_{K}$. If $2 \mid q_{K}$, then it is possible that $F$ is not a 
separable extension. However, in this case we have $K=\left\{\alpha^{2}: \alpha \in F\right\}$ and $g_{F}=g_{K}$ by [11, Proposition III.9.2]. This completely determines the unique such quadratic extension, so for our purposes here we will only consider separable extensions from now on.

Both [11, Chapter III] and [13, Chapter VIII] contain all the information we require concerning the different and discriminant. Here we simply note that every extension $F$ of a given function field $K$ has a canonically given divisor $\operatorname{Diff}_{K}(F) \in \operatorname{Div}(F)$ called the different. The discriminant $\operatorname{Disc}_{K}(F)$ is the "relative norm" of the different:

$$
\operatorname{Disc}_{K}(F)=\sum_{v \in M(K)}\left(\sum_{\substack{w \in M(F) \\ w \mid v}} \operatorname{ord}_{w}\left(\operatorname{Diff}_{K}(F)\right) \operatorname{deg}_{F}(w) / \operatorname{deg}_{K}(v)\right) \cdot v \in \operatorname{Div}(K) .
$$

One readily verifies via the definitions that

$$
\operatorname{deg}_{K}\left(\operatorname{Disc}_{K}(F)\right)=\operatorname{deg}_{F}\left(\operatorname{Diff}_{K}(F)\right)
$$

(This is true for the relative norm of any divisor in $\operatorname{Div}(F)$, of course, not just the different.)

Proposition 1. Suppose $K$ is a function field with field of constants $\mathbb{F}_{q}$ and $F$ is a separable quadratic extension of $K$ with $q_{F}=q$. Then the different $\operatorname{Diff}_{K}(F) \in \operatorname{Div}(F)$ is an effective divisor with

$$
2\left(g_{F}-1\right)=4\left(g_{K}-1\right)+\operatorname{deg}_{F}\left(\operatorname{Diff}_{K}(F)\right)=4\left(g_{K}-1\right)+\operatorname{deg}_{K}\left(\operatorname{Disc}_{K}(F)\right) .
$$

If $2 \nmid q$, then both $\operatorname{Diff}_{K}(F)$ and $\operatorname{Disc}_{K}(F)$ are square-free effective divisors of even degree. If $2 \mid q$, then $\operatorname{Diff}_{K}(F) \in 2 \operatorname{Div}(F)$ and $\operatorname{Disc}_{K}(F) \in 2 \operatorname{Div}(K)$. For any effective divisor $\mathfrak{A} \in \operatorname{Div}(K)$, we have $\chi(F / \mathfrak{A}) \neq 0$ if and only if $\operatorname{Disc}_{K}(F)$ and $\mathfrak{A}$ have disjoint support.

Proof. The equation involving the genera is simply an instance of the Hurwitz Genus Formula ([11, Theorem III.4.12]) and (3). Dedekind's Different Theorem [11, Theorem III.5.1] implies that $\operatorname{ord}_{w}\left(\operatorname{Diff}_{K}(F)\right)=1$ for all $w \in \operatorname{Supp}\left(\operatorname{Diff}_{K}(F)\right)$ when $q$ is odd. We then have $\operatorname{ord}_{v}\left(\operatorname{Disc}_{K}(F)\right)=1$ for all $v \in \operatorname{Supp}\left(\operatorname{Disc}_{K}(F)\right)$ since in this case $F$ is a Kummer extension of $K$ (see [11, Proposition III.7.3], for example). In the case where $q$ is even $F$ is an Artin-Schreier extension of $K$; we have $\operatorname{Diff}_{K}(F) \in 2 \operatorname{Div}(F)$ and $\operatorname{Disc}_{K}(F) \in 2 \operatorname{Div}(K)$ by [11, Proposition III.7.8]. The last part of the Proposition follows from Dedekind's Theorem and [11, Proposition III.7.8]. 
Via Proposition 1, we see that our sums (2) may be realized as follows:

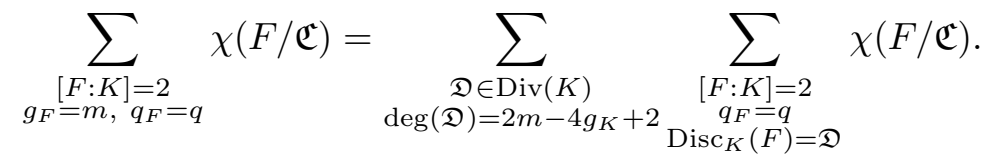

It remains to determine which divisors $\mathfrak{D} \in \operatorname{Div}(K)$ are of the form $\mathfrak{D}=\operatorname{Disc}_{K}(F)$ for some separable quadratic extension $F$ (beyond that which is stated in Proposition 1), and how many quadratic extensions $F$ have $\operatorname{Disc}_{K}(F)=\mathfrak{D}$ for a given effective divisor $\mathfrak{D} \in \operatorname{Div}(K)$. We introduce a bit more notation.

Notation. Let $\mathfrak{D} \in \operatorname{Div}(K)$ be a square-free effective divisor of even degree if $q_{K}$ is odd, and simply a non-zero effective divisor if $q_{K}$ is even. Set $S(\mathfrak{D})$ to be the set of separable quadratic extensions $F$ of $K$ with $q_{F}=q_{K}$ and $\operatorname{Disc}_{K}(F)=\mathfrak{D}$ if $q_{K}$ is odd, and $\operatorname{Disc}_{K}(F)=2 \mathfrak{D}$ if $q_{K}$ is even. Set $N(\mathfrak{D})=\# S(\mathfrak{D})$.

We will consider the cases of odd and even characteristic separately.

Suppose first that $K$ is a function field with $q_{K}=q$ odd. Since the characteristic of $K$ is odd, every quadratic extension $F$ of $K$ is of the form $F=K(y)$, where $y^{2}=\omega \in K^{\times}$and $\omega \neq \alpha^{2}$ for all $\alpha \in K$. We say $\omega$ generates $F$ in this case. One readily sees that $\omega$ and $\omega^{\prime}$ generate the same quadratic extension if and only if $\omega^{\prime}=\alpha^{2} \omega$ for some $\alpha \in K^{\times}$.

Proposition 2. Suppose $K$ is a function field with $q_{K}=q$ odd. Define $\theta: \operatorname{Div}(K) \rightarrow \operatorname{Div}(K) \otimes$ $\mathbb{Z} / 2 \mathbb{Z}$ by $\theta\left(\sum n_{v} \cdot v\right)=\sum \pi\left(n_{v}\right) \cdot v$, where $\pi: \mathbb{Z} \rightarrow \mathbb{Z} / 2 \mathbb{Z}$ is the canonical map. Let $\psi$ be the multiplication by 2 map on the group of divisor classes of degree 0 . The image under $\theta$ of the subset of square-free effective divisors of even degree is a subgroup $G_{K}$ of $\operatorname{Div}(K) \otimes \mathbb{Z} / 2 \mathbb{Z}$. The image under $\theta$ of the set of discriminants $\operatorname{Disc}_{K}(F)$ of quadratic extensions $F \supset K$ with $q_{F}=q$ together with the zero divisor is a subgroup of index $\# \operatorname{ker}(\psi)$ in $G_{K}$. Further, $N(\mathfrak{D})=2 \# \operatorname{ker}(\psi)$ whenever $S(\mathfrak{D}) \neq \emptyset$.

Proof. In what follows, $\operatorname{Div}_{0}(K)$ denotes the group of divisors of degree 0 and $\mathcal{P}_{K}$ denotes the group of principal divisors, so that $\operatorname{Div}_{0}(K) / \mathcal{P}_{K}$ is a group of order $J_{K}$. Set $G_{K}=\theta\left(\operatorname{Div}_{0}(K)\right)$.

For a $\mathfrak{D} \in \operatorname{Div}_{0}(K)$, write $\mathfrak{D}=\mathfrak{D}^{\prime}+2 \mathfrak{D}^{\prime \prime}$, where $\mathfrak{D}^{\prime}$ is a square-free effective divisor. Then $\theta(\mathfrak{D})=$ $\theta\left(\mathfrak{D}^{\prime}\right)$ and $0=\operatorname{deg}\left(\mathfrak{D}^{\prime}\right)+2 \operatorname{deg}\left(\mathfrak{D}^{\prime \prime}\right) \equiv \operatorname{deg}\left(\mathfrak{D}^{\prime}\right) \bmod 2$. On the other hand, let $\mathfrak{D}=v_{1}+\cdots+v_{n}$ be 
a square-free effective divisor of even degree, say $\operatorname{deg}(\mathfrak{D})=2 m$. Choose a positive integer $k$ and a place $v \notin \operatorname{Supp}(\mathfrak{D})$ with $k \operatorname{deg}\left(v_{1}\right)+m=\operatorname{deg}(v)$. Then $\mathfrak{E}=(2 k+1) v_{1}+v_{2}+\cdots+v_{n}-2 v \in \operatorname{Div}_{0}(K)$ with $\theta(\mathfrak{E})=\mathfrak{D}$. This proves the first part of the proposition.

Next, for a quadratic extension $F \supset K$ with $q_{F}=q$ generated by $\omega$ we have $\theta\left(\operatorname{Disc}_{K}(K)\right)=$ $\theta(\operatorname{div}(\omega))$, where $\operatorname{div}(\omega)$ is the principal ideal associated with $\omega: \operatorname{div}(\omega)=\sum \operatorname{ord}_{v}(\omega) \cdot v$. $($ See $[11$, Proposition III.7.3], for example.) Let $\psi: \operatorname{Div}_{0}(K) / \mathcal{P}_{K} \rightarrow \operatorname{Div}_{0}(K) / \mathcal{P}_{K}$ be the multiplication by 2 map: $\psi\left(\mathfrak{A}+\mathcal{P}_{K}\right)=2 \mathfrak{A}+\mathcal{P}_{K}$. Since the kernel of $\theta$ restricted to $\operatorname{Div}_{0}(K)$ is clearly $2 \operatorname{Div}_{0}(K)$, we see that the index

$$
\left[G_{K}: \theta\left(\mathcal{P}_{K}\right)\right]=\left[\theta\left(\operatorname{Div}_{0}(K)\right): \theta\left(\mathcal{P}_{K}\right)\right]=\frac{\# \operatorname{Div}_{0}(K) / \mathcal{P}_{K}}{\# \psi\left(\operatorname{Div}_{0}(K) / \mathcal{P}_{K}\right)}=\frac{J_{K}}{J_{K} / \# \operatorname{ker}(\psi)}=\# \operatorname{ker}(\psi)
$$

Set $n=\# \operatorname{ker}(\psi)$, let $\mathfrak{A}_{1}=0, \ldots, \mathfrak{A}_{n} \in \operatorname{Div}_{0}(K)$ be representatives of $\operatorname{ker}(\psi)$ and let $\omega_{1}=$ $1, \ldots, \omega_{n} \in K^{\times}$with $\operatorname{div}\left(\omega_{i}\right)=2 \mathfrak{A}_{i}$ for $i=1, \ldots, n$. Then $\omega, \omega^{\prime} \in K$ generate quadratic extensions $F$ and $F^{\prime}$ with $\operatorname{Disc}_{K}(F)=\operatorname{Disc}_{K}\left(F^{\prime}\right)$ if and only if $\omega^{\prime}=a \omega_{i} \alpha^{2} \omega$ for some $a \in \mathbb{F}_{q}^{\times}, 1 \leq i \leq n$ and $\alpha \in K^{\times}$. Finally, if $\omega / \omega^{\prime} \in \mathbb{F}_{q}^{\times}$and $\omega / \omega^{\prime}$ isn't a square (in $K$ ), then $F \neq F^{\prime}$, since otherwise there is an $x \in F=F^{\prime}$ with $x^{2} \in \mathbb{F}_{q}^{\times}$a non-square (in $\mathbb{F}_{q}$ ) so that $q_{F}=q^{2}$, contradicting our hypothesis. Since the squares in $\mathbb{F}_{q}^{\times}$form a subgroup of index 2 in $\mathbb{F}_{q}^{\times}$, we see that $N(\mathfrak{D})=2 n$ whenever $S(\mathfrak{D}) \neq \emptyset$.

We now turn to the case of characteristic two. Again our goal is to determine $S(\mathfrak{D})$ and $N(\mathfrak{D})$; this is achieved in Proposition 3. As opposed to the case of odd characteristic where $N(\mathfrak{D})$ is easily determined but $S(\mathfrak{D})$ is somewhat mysterious, we find that $S(\mathfrak{D})$ is almost always non-empty (see Lemma 5 below) but determining $N(\mathfrak{D})$ is non-trivial in characteristic 2 . Throughout the remainder of this section $K$ is a fixed function field with $q_{K}=q$ even.

Suppose $\omega \in K$ with $\omega \neq \alpha^{2}+\alpha$ for all $\alpha \in K$. We then have a quadratic extension $F=K(y)$ where $y^{2}+y+\omega=0$; we say $\omega$ generates the extension $F$. All (separable) quadratic extensions $F$ arise in this manner. Similar to the case of odd characteristic, one readily verifies that $\omega$ and $\omega^{\prime}$ generate the same quadratic extension if and only if $\omega^{\prime}=\omega+\alpha^{2}+\alpha$ for some $\alpha \in K$.

For a quadratic extension $F$ generated by $\omega, \operatorname{Disc}_{K}(F)=\sum\left(n_{v}+1\right) \cdot v$ has the following properties 
(see [11, Proposition III.7.8]):

$$
\begin{aligned}
& \text { i) } n_{v} \geq-1 \quad \text { for all } v \in M(K) \text {; } \\
& \text { ii) } n_{v} \equiv 1 \bmod 2 \quad \text { for all } v \in M(K) \text {; } \\
& \text { iii) }-n_{v}=\max _{\alpha \in K}\left\{\operatorname{ord}_{v}\left(\omega+\alpha^{2}+\alpha\right)\right\} \quad \text { if } n_{v}>0 \text {; } \\
& \text { iv) } n_{v}=-1 \quad \text { if } \operatorname{ord}_{v}\left(\omega+\alpha^{2}+\alpha\right) \geq 0 \text { for some } \alpha \in K \text {. }
\end{aligned}
$$

Obviously $n_{v}>0$ only if $\operatorname{ord}_{v}(\omega)<0$, and $\operatorname{Disc}_{K}(F) \in 2 \operatorname{Div}(K)$ as mentioned in Proposition 1 . We have the following simple result.

Lemma 3. Suppose $\omega \in K$. If $\operatorname{ord}_{v}(\omega)<0$ and odd for any place $v \in M(K)$, then $\omega$ generates a quadratic extension $F$ with $\operatorname{ord}_{v}\left(\operatorname{Disc}_{K}(F)\right)=1-\operatorname{ord}_{v}(\omega)$.

Proof. If $\alpha \in K$ with $\operatorname{ord}_{v}(\alpha) \geq 0$, then $\operatorname{ord}_{v}\left(\alpha^{2}+\alpha\right) \geq 0$ and $\operatorname{ord}_{v}(\omega)=\operatorname{ord}_{v}\left(\omega+\alpha^{2}+\alpha\right)$. If $\operatorname{ord}_{v}(\alpha)<0$, then $\operatorname{ord}_{v}\left(\alpha^{2}+\alpha\right)=\operatorname{ord}_{v}\left(\alpha^{2}\right) \in 2 \mathbb{Z}$. This implies that $\operatorname{ord}_{v}\left(\omega+\alpha^{2}+\alpha\right)=$ $\min \left\{\operatorname{ord}_{v}(\omega), \operatorname{ord}_{v}\left(\alpha^{2}\right)\right\}$. The lemma follows from this and (4).

Lemma 4. Suppose $\mathfrak{A}, \mathfrak{B} \in \operatorname{Div}(K)$ are effective divisors with $(\mathfrak{A}, \mathfrak{B})=0$. Then

$$
\begin{aligned}
& \#\left\{\alpha \in L(\mathfrak{A}+\mathfrak{B}): \operatorname{ord}_{v}(\alpha)=-\operatorname{ord}_{v}(\mathfrak{A}) \text { for all } v \in \operatorname{Supp}(\mathfrak{A})\right\} \\
& =q^{\operatorname{deg}(\mathfrak{B})+1-g_{K}} \phi(\mathfrak{A}) \\
& +\sum_{\substack{0 \leq \mathfrak{C} \leq \mathfrak{A} \\
\operatorname{deg}(\mathfrak{A}+\mathfrak{B}-\mathfrak{C})<2 g_{K}-1}} \mu(\mathfrak{C})\left(q^{l(\mathfrak{A}+\mathfrak{B}-\mathfrak{C})}-q^{\operatorname{deg}(\mathfrak{A}+\mathfrak{B}-\mathfrak{C})+1-g_{K}}\right) .
\end{aligned}
$$

In particular, if $\operatorname{deg}(\mathfrak{B}) \geq 2 g_{K}-1$, then

$$
\#\left\{\alpha \in L(\mathfrak{A}+\mathfrak{B}): \operatorname{ord}_{v}(\alpha)=-\operatorname{ord}_{v}(\mathfrak{A}) \text { for all } v \in \operatorname{Supp}(\mathfrak{A})\right\}=q^{\operatorname{deg}(\mathfrak{B})+1-g_{K}} \phi(\mathfrak{A}) .
$$

Proof. We first express $L(\mathfrak{A}+\mathfrak{B})$ as a disjoint union:

$$
L(\mathfrak{A}+\mathfrak{B})=\bigcup_{0 \leq \mathfrak{C} \leq \mathfrak{A}}\left\{\alpha \in L(\mathfrak{A}-\mathfrak{C}+\mathfrak{B}): \operatorname{ord}_{v}(\alpha)=-\operatorname{ord}_{v}(\mathfrak{A}-\mathfrak{C}) \text { for all } v \in \operatorname{Supp}(\mathfrak{A}-\mathfrak{C})\right\}
$$

For notational convenience, temporarily set

$$
L^{\prime}(\mathfrak{A}-\mathfrak{C}, \mathfrak{B})=\left\{\alpha \in L(\mathfrak{A}-\mathfrak{C}+\mathfrak{B}): \operatorname{ord}_{v}(\alpha)=-\operatorname{ord}_{v}(\mathfrak{A}-\mathfrak{C}) \text { for all } v \in \operatorname{Supp}(\mathfrak{A}-\mathfrak{C})\right\}
$$


so that

$$
q^{l(\mathfrak{A}+\mathfrak{B})}=\sum_{0 \leq \mathfrak{C} \leq \mathfrak{A}} \# L^{\prime}(\mathfrak{A}-\mathfrak{C}, \mathfrak{B}) .
$$

We use this together with $(1)$ to get $($ with $\mathfrak{E}=\mathfrak{C}+\mathfrak{D})$

$$
\begin{aligned}
\sum_{0 \leq \mathfrak{C} \leq \mathfrak{A}} \mu(\mathfrak{C}) q^{l(\mathfrak{A}+\mathfrak{B}-\mathfrak{C})} & =\sum_{0 \leq \mathfrak{C} \leq \mathfrak{A}} \mu(\mathfrak{C}) \sum_{0 \leq \mathfrak{D} \leq \mathfrak{A}-\mathfrak{C}} \# L^{\prime}(\mathfrak{A}-\mathfrak{C}-\mathfrak{D}, \mathfrak{B}) \\
& =\sum_{0 \leq \mathfrak{E} \leq \mathfrak{A}} \# L^{\prime}(\mathfrak{A}-\mathfrak{E}, \mathfrak{B}) \sum_{0 \leq \mathfrak{C} \leq \mathfrak{E}} \mu(\mathfrak{C}) \\
& =\# L^{\prime}(\mathfrak{A}, \mathfrak{B}) \\
& =\#\left\{\alpha \in L(\mathfrak{A}+\mathfrak{B}): \operatorname{ord}_{v}(\alpha)=-\operatorname{ord}_{v}(\mathfrak{A}) \text { for all } v \in \operatorname{Supp}(\mathfrak{A})\right\}
\end{aligned}
$$

Now by the Riemann-Roch Theorem we have

$$
\begin{aligned}
\sum_{0 \leq \mathfrak{C} \leq \mathfrak{A}} \mu(\mathfrak{C}) q^{l(\mathfrak{A}+\mathfrak{B}-\mathfrak{C})}= & \sum_{0 \leq \mathfrak{C} \leq \mathfrak{A}} \mu(\mathfrak{C}) q^{\operatorname{deg}(\mathfrak{A}+\mathfrak{B}-\mathfrak{C})+1-g_{K}} \\
& +\sum_{\substack{0 \leq \mathfrak{C} \leq \mathfrak{A} \\
\operatorname{deg}(\mathfrak{A}+\overline{\mathfrak{B}}-\mathfrak{C})<2 g-1}} \mu(\mathfrak{C})\left(q^{l(\mathfrak{A}+\mathfrak{B}-\mathfrak{C})}-q^{\operatorname{deg}(\mathfrak{A}+\mathfrak{B}-\mathfrak{C})+1-g_{K}}\right)
\end{aligned}
$$

Since

$$
\begin{aligned}
\sum_{0 \leq \mathfrak{C} \leq \mathfrak{A}} \mu(\mathfrak{C}) q^{\operatorname{deg}(\mathfrak{A}+\mathfrak{B}-\mathfrak{C})+1-g_{K}} & =q^{\operatorname{deg}(\mathfrak{B})+1-g_{K}} q^{\operatorname{deg}(\mathfrak{A})} \sum_{0 \leq \mathfrak{C} \leq \mathfrak{A}} \mu(\mathfrak{C}) q^{-\operatorname{deg}(\mathfrak{C})} \\
& =q^{\operatorname{deg}(\mathfrak{B})+1-g_{K}} q^{\operatorname{deg}(\mathfrak{A})} \prod_{v \in \operatorname{Supp}(\mathfrak{A})} 1-q^{-\operatorname{deg}(v)} \\
& =q^{\operatorname{deg}(\mathfrak{B})+1-g_{K}} \phi(\mathfrak{A})
\end{aligned}
$$

the lemma follows.

Lemma 5. Suppose $\mathfrak{D} \in \operatorname{Div}(K)$ is a non-zero effective divisor and $\omega \in L^{\prime}\left(\mathfrak{D}_{1}+2 \mathfrak{D}_{2}\right)$. Then $\omega \neq \alpha^{2}+\alpha$ for all $\alpha \in K$ and $\omega$ generates a quadratic extension $F$ with $\operatorname{Disc}_{K}(F)=2 \mathfrak{D}$. Further, $\omega, \omega^{\prime} \in L^{\prime}\left(\mathfrak{D}_{1}+2 \mathfrak{D}_{2}\right)$ both generate the same quadratic extension $F$ if and only if $\omega^{\prime}=\omega+\beta^{2}+\beta$ for some $\beta \in L\left(\mathfrak{D}_{2}\right)$. There are precisely

$$
\begin{aligned}
\frac{2 \# L^{\prime}\left(\mathfrak{D}_{1}+2 \mathfrak{D}_{2}\right)}{q^{l\left(\mathfrak{D}_{2}\right)}}= & \frac{2 \phi(\mathfrak{D})}{q^{l\left(\mathfrak{W}_{K}-\mathfrak{D}_{2}\right)}} \\
& +\frac{2}{q^{l\left(\mathfrak{D}_{2}\right)}} \sum_{\substack{0 \leq \mathfrak{C} \leq \mathfrak{D}_{1}+\mathfrak{D}_{2} \\
\operatorname{deg}\left(\mathfrak{D}_{1}+2 \mathfrak{D}_{2}-\mathfrak{C}\right)<2 g_{K}-1}} \mu(\mathfrak{C})\left(q^{l\left(\mathfrak{D}_{1}+2 \mathfrak{D}_{2}-\mathfrak{C}\right)}-q^{\operatorname{deg}\left(\mathfrak{D}_{1}+2 \mathfrak{D}_{2}-\mathfrak{C}\right)+1-g_{K}}\right)
\end{aligned}
$$

such extensions $F$, where $\mathfrak{W}_{K} \in \operatorname{Div}(K)$ is any divisor in the canonical class. In particular, if $\operatorname{deg}\left(\mathfrak{D}_{2}\right) \geq 2 g_{K}-1$, then there are precisely $2 \phi(\mathfrak{D})$ such extensions. 
Proof. Let $v$ be any place in the support of $\mathfrak{D}_{1}+2 \mathfrak{D}_{2}$. Then $\operatorname{ord}_{v}(\omega)<0$ and odd. As in the proof of Lemma 1 we see that $\operatorname{ord}_{v}(\omega) \neq \operatorname{ord}_{v}\left(\alpha^{2}+\alpha\right)$ for all $\alpha \in K$, so that $\omega \neq \alpha^{2}+\alpha$ for all $\alpha \in K$. By construction $\operatorname{ord}_{v}(\omega)<0$ only if $\operatorname{ord}_{v}(\omega)$ is odd. Thus $\operatorname{ord}_{v}\left(\operatorname{Disc}_{K}(F)\right)=1-\operatorname{ord}_{v}(\omega)=2 \operatorname{ord}_{v}(\mathfrak{D})$ for all places $v$ in the support of $\mathfrak{D}$ by Lemma 3. Moreover, by construction $\operatorname{ord}_{v}(\omega) \geq 0$ for all places $v$ not in the support of $\mathfrak{D}$. Thus $\operatorname{Disc}_{K}(F)=2 \mathfrak{D}$ by $(4)$.

Now suppose $\omega, \omega^{\prime} \in L^{\prime}\left(\mathfrak{D}_{1}+2 \mathfrak{D}_{2}\right)$ generate the same quadratic extension $F$. Then as noted above, $\omega^{\prime}=\omega+\beta^{2}+\beta$ for some $\beta \in K$. If $\operatorname{ord}_{v}(\beta)<0$, then as shown in the proof of Lemma 3 , $\operatorname{ord}_{v}\left(\omega^{\prime}\right)=\operatorname{ord}_{v}\left(\omega+\beta^{2}+\beta\right)=\min \left\{\operatorname{ord}_{v}(\omega), 2 \operatorname{ord}_{v}(\beta)\right\}$. Since $\operatorname{ord}_{v}\left(\omega^{\prime}\right)=\operatorname{ord}_{v}(\omega)$ for all places $v$ in the support of $\mathfrak{D}$ and $\operatorname{ord}_{v}\left(\omega^{\prime}\right), \operatorname{ord}_{v}(\omega) \geq 0$ for all other places, we see that $v$ here must be in the support of $\mathfrak{D}$ and $2 \operatorname{ord}_{v}(\beta)>\operatorname{ord}_{v}(\omega)=-1-2 \operatorname{ord}_{v}\left(\mathfrak{D}_{2}\right)$. Thus, $\operatorname{ord}_{v}(\beta) \geq-\operatorname{ord}_{v}\left(\mathfrak{D}_{2}\right)$ for all places $v$ in the support of $\mathfrak{D}$, so that $\beta \in L\left(\mathfrak{D}_{2}\right)$. Conversely, if $\beta \in L\left(\mathfrak{D}_{2}\right)$, then $\operatorname{ord}_{v}\left(\omega+\beta^{2}+\beta\right)=$ $\operatorname{ord}_{v}(\omega)$ for all places $v$ in the support of $\mathfrak{D}$ and $\operatorname{ord}_{v}\left(\omega+\beta^{2}+\beta\right) \geq 0$ for all other places, so that $\omega^{\prime}=\omega+\beta^{2}+\beta \in L^{\prime}\left(\mathfrak{D}_{1}+2 \mathfrak{D}_{2}\right)$ and both generate the same quadratic extension.

Finally, we note that the mapping $\beta \mapsto \beta^{2}+\beta$ is two-to-one on $L\left(\mathfrak{D}_{2}\right)$ since $\beta_{1}^{2}+\beta_{1}=\beta_{2}^{2}+\beta_{2}$ if and only if $\left(\beta_{1}+\beta_{2}\right)^{2}+\beta_{1}+\beta_{2}=0$, i.e., $\beta_{1}+\beta_{2}=0$ or 1 . Thus, there are $\frac{2 \# L^{\prime}\left(\mathfrak{D}_{1}+2 \mathfrak{D}_{2}\right)}{q^{l\left(\mathfrak{D}_{2}\right)}}$ extensions $F$ with a generator in $L^{\prime}\left(\mathfrak{D}_{1}+2 \mathfrak{D}_{2}\right)$. The remainder of the lemma now follows from Lemma 4 , upon noting that

$$
\begin{aligned}
\frac{q^{1-g_{K}} \phi\left(\mathfrak{D}_{1}+2 \mathfrak{D}_{2}\right)}{q^{l\left(\mathfrak{D}_{2}\right)}} & =\frac{q^{\operatorname{deg}\left(\mathfrak{D}_{2}\right)+1-g_{K}} \phi\left(\mathfrak{D}_{1}+\mathfrak{D}_{2}\right)}{q^{\operatorname{deg}\left(\mathfrak{D}_{2}\right)+1-g_{K}+l\left(\mathfrak{W}_{K}-\mathfrak{D}_{2}\right)}} \\
& =\frac{\phi(\mathfrak{D})}{q^{l\left(\mathfrak{W}_{K}-\mathfrak{D}_{2}\right)}} .
\end{aligned}
$$

Lemma 6. Suppose $\mathfrak{D}$ is an effective divisor and $F$ is a quadratic extension of $K$ with $\operatorname{Disc}_{K}(F)=$ $2 \mathfrak{D}$. Suppose $S$ is a finite non-empty set of places. Then there is a generator of $F$ in

$$
\left\{\alpha \in L\left(\mathfrak{D}_{1}+2 \mathfrak{D}_{2}+2 \mathfrak{A}\right): \operatorname{ord}_{v}(\alpha)=1-2 \operatorname{ord}_{v}(\mathfrak{D}) \text { for all } v \in \operatorname{Supp}(\mathfrak{D}) \backslash S\right\}
$$

for some effective divisor $\mathfrak{A}$ with $\operatorname{Supp}(\mathfrak{A}) \subseteq S$.

Proof. For notational convenience set $n_{v}=\operatorname{ord}_{v}\left(\mathfrak{D}_{1}+2 \mathfrak{D}_{2}\right)=2 \operatorname{ord}_{v}(\mathfrak{D})-1$ for $v \in \operatorname{Supp}(\mathfrak{D})$. Let $\omega$ be a generator of $F$ and set

$$
M_{1}(\omega)=\left\{v \in \operatorname{Supp}(\mathfrak{D}): \operatorname{ord}_{v}(\omega) \neq-n_{v}\right\}
$$


If $M_{1}(\omega)$ is not empty, then we claim that there is a generator $\omega^{\prime}$ of $F$ with $\# M_{1}\left(\omega^{\prime}\right)<\# M_{1}(\omega)$. To see why, let $v^{\prime} \in \operatorname{Supp}(\mathfrak{D})$ with $\operatorname{ord}_{v^{\prime}}(\omega) \neq-n_{v^{\prime}}$. By (4) there is an $\alpha \in K$ with $\operatorname{ord}_{v^{\prime}}\left(\omega+\alpha^{2}+\alpha\right)=$ $-n_{v^{\prime}}$. By the Weak Approximation Theorem [11, Theorem I.3.1] there is a $\beta \in K$ with ord ${ }_{v^{\prime}}(\beta)=0$ and $\operatorname{ord}_{v}(\alpha+\beta)=0$ for all other $v \in \operatorname{Supp}(\mathfrak{D})$. Then $\omega^{\prime}=\omega+(\alpha+\beta)^{2}+\alpha+\beta$ is a generator of $F$ with $\operatorname{ord}_{v^{\prime}}\left(\omega^{\prime}\right)=-n_{v}$ and $\operatorname{ord}_{v}\left(\omega^{\prime}\right) \geq \min \left\{\operatorname{ord}_{v}(\omega), 0\right\}$ for all other places $v \in \operatorname{Supp}(\mathfrak{D})$, so that $\# M_{1}\left(\omega^{\prime}\right)<\# M_{1}(\omega)$. Repeatedly applying this claim yields a generator $\omega$ of $F$ with $\operatorname{ord}_{v}(\omega)=-n_{v}$ for all places $v \in \operatorname{Supp}(\mathfrak{D})$.

By Lemma 3, if $\operatorname{ord}_{v}(\omega)<0$ and $v \notin \operatorname{Supp}(\mathfrak{D})$, then $\operatorname{ord}_{v}(\omega)$ must be even. Set

$$
M_{2}(\omega):=\left\{v \in M(K): \operatorname{ord}_{v}(\omega)<0, v \notin \operatorname{Supp}(\mathfrak{D}) \cup S\right\}
$$

If $M_{2}(\omega)$ is empty, we are done. If not, we claim there is a generator $\omega^{\prime}$ of $F$ with $\# M_{2}\left(\omega^{\prime}\right)<$ $\# M_{2}(\omega)$ and $\operatorname{ord}_{v}\left(\omega^{\prime}\right)=\operatorname{ord}_{v}(\omega)$ for all $v \in \operatorname{Supp}(\mathfrak{D}) \backslash S$. To see why, let $v^{\prime} \in M_{2}(\omega)$. By (4) there is an $\alpha \in K$ with $\operatorname{ord}_{v^{\prime}}\left(\omega+\alpha^{2}+\alpha\right) \geq 0$. By the Strong Approximation Theorem [11 Theorem I.6.4], there is a $\beta \in K$ such that $\operatorname{ord}_{v}(\alpha+\beta)=0$ for all places $v \in M(K)$ with $\operatorname{ord}_{v}(\alpha)<0$ (note that $v^{\prime}$ is such a place) and $\operatorname{ord}_{v}(\beta) \geq 0$ for any other places $v$ in the complement of $S$. We set $\omega^{\prime}=\omega+(\alpha+\beta)^{2}+\alpha+\beta$. Then $\operatorname{ord}_{v}\left(\omega^{\prime}\right)=\operatorname{ord}_{v}(\omega)$ for all places $v$ in the intersection of $\operatorname{Supp}(\mathfrak{D})$ and the complement of $S$, $\operatorname{ord}_{v^{\prime}}\left(\omega^{\prime}\right) \geq 0$ and $\operatorname{ord}_{v}\left(\omega^{\prime}\right) \geq \min \left\{\operatorname{ord}_{v}(\omega), 0\right\}$ for all other places $v$ in the complement of $S$. Since the original $M_{2}(\omega)$ is necessarily a finite set, repeatedly applying this claim yields the desired generator.

Lemma 7. Suppose $\mathfrak{D}$ and $\mathfrak{A}$ are effective divisors with $(\mathfrak{D}, \mathfrak{A})=0$. Suppose $F$ is a quadratic extension of $K$ and the set

$$
\left\{\alpha \in L\left(\mathfrak{D}_{1}+2 \mathfrak{D}_{2}+2 \mathfrak{A}\right): \operatorname{ord}_{v}(\alpha)=1-\operatorname{2ord}_{v}(\mathfrak{D}) \text { for all } v \in \operatorname{Supp}(\mathfrak{D})\right\}
$$

contains a generator $\omega$ of $F$. Then $\operatorname{Disc}_{K}(F)=2 \mathfrak{D}+2 \mathfrak{B}$ for some effective divisor $\mathfrak{B} \leq \mathfrak{A}$, and $\omega^{\prime} \in L\left(\mathfrak{D}_{1}+2 \mathfrak{D}_{2}+2 \mathfrak{A}\right)$ generates $F$ if and only if $\omega^{\prime}=\omega+\alpha^{2}+\alpha$ for some $\alpha \in L\left(\mathfrak{D}_{2}+\mathfrak{A}\right)$. In particular, there are exactly $q^{l\left(\mathfrak{D}_{2}+\mathfrak{A}\right)} / 2$ generators of $F$ in this set.

Proof. Suppose $F$ is generated by an $\omega$ in the given set. By (4), $\operatorname{Supp}\left(\operatorname{Disc}_{K}(F)\right) \subseteq \operatorname{Supp}(\mathfrak{D}) \cup$ $\operatorname{Supp}(\mathfrak{A})$. By Lemma 3 and the definitions of $\mathfrak{D}_{1}$ and $\mathfrak{D}_{2}, \operatorname{ord}_{v}\left(\operatorname{Disc}_{K}(F)\right)=2 \operatorname{ord}(\mathfrak{D})$ for all $v \in$ 
$\operatorname{Supp}(\mathfrak{D})$, so that $\operatorname{Disc}_{K}(F) \supseteq 2 \mathfrak{D}$. Also by $(4), \operatorname{2ord}_{v}\left(\operatorname{Disc}_{K}(F)\right) \leq 2 \operatorname{ord}_{v}(\mathfrak{A})$ for all $v \in \operatorname{Supp}(\mathfrak{A})$. This proves the first part of the lemma.

Set $n_{v}=2 \operatorname{ord}_{v}(\mathfrak{D})-1$ for $v \in \operatorname{Supp}(\mathfrak{D})$. Suppose $\omega^{\prime} \in L\left(\mathfrak{D}_{1}+2 \mathfrak{D}_{2}+2 \mathfrak{A}\right)$ generates $F$. Then $\omega^{\prime}=\omega+\alpha^{2}+\alpha$ for some $\alpha \in K$ and $\operatorname{ord}_{v}\left(\omega^{\prime}\right) \geq-n_{v}$ for all places $v \in \operatorname{Supp}(\mathfrak{D})$. By (4), this implies that $\operatorname{ord}_{v}\left(\omega^{\prime}\right)=-n_{v}$ for all places $v \in \operatorname{Supp}(\mathfrak{D})$. If $\operatorname{ord}_{v}(\alpha)<-\operatorname{ord}_{v}\left(\mathfrak{D}_{2}\right)$ for some $v \in \operatorname{Supp}(\mathfrak{D})$, then $\operatorname{ord}_{v}\left(\alpha^{2}+\alpha\right)=2 \operatorname{ord}_{v}(\alpha) \leq-2 \operatorname{ord}_{v}\left(\mathfrak{D}_{2}\right)-2=n_{v}-1$ so that $\operatorname{ord}_{v}\left(\omega^{\prime}\right)<-n_{v} . \operatorname{Thus}_{\operatorname{ord}}(\alpha) \geq$ $-\operatorname{ord}_{v}\left(\mathfrak{D}_{2}\right)$ for all places $v \in \operatorname{Supp}(\mathfrak{D})$. Similarly, if $v \in \operatorname{Supp}(\mathfrak{A})$ and $\operatorname{ord}_{v}(\alpha)<-\operatorname{ord}_{v}(\mathfrak{A})$, then $\operatorname{ord}_{v}\left(\alpha^{2}+\alpha\right)=2 \operatorname{ord}_{v}(\alpha)<-2 \operatorname{ord}_{v}(\mathfrak{A})$ and $\operatorname{ord}_{v}\left(\omega+\alpha^{2}+\alpha\right)=2 \operatorname{ord}_{v}(\alpha)<-2 \operatorname{ord}_{v}(\mathfrak{A})$ since $\operatorname{ord}_{v}(\omega) \geq-2 \operatorname{ord}_{v}(\mathfrak{A})$. Thus $\operatorname{ord}_{v}(\alpha) \geq-\operatorname{ord}_{v}(\mathfrak{A})$ for all places $v \in \operatorname{Supp}(\mathfrak{A})$ and $\alpha \in L\left(\mathfrak{D}_{2}+\mathfrak{A}\right)$.

Conversely, suppose $\alpha \in L\left(\mathfrak{D}_{2}+\mathfrak{A}\right)$ and let $\omega^{\prime}=\omega+\alpha^{2}+\alpha$. Then for all places $v \in \operatorname{Supp}\left(\mathfrak{D}_{2}\right)$ we have $\operatorname{ord}_{v}\left(\alpha^{2}+\alpha\right) \geq-2 \operatorname{ord}_{v}\left(\mathfrak{D}_{2}\right)>-n_{v}=\operatorname{ord}_{v}(\omega)$ so that $\operatorname{ord}_{v}\left(\omega^{\prime}\right)=\operatorname{ord}_{v}(\omega)=-n_{v}$. For all places $v \in \operatorname{Supp}(\mathfrak{A})$ we have $\operatorname{ord}_{v}\left(\alpha^{2}+\alpha\right) \geq-2 \operatorname{ord}_{v}(\mathfrak{A})$ so that $\operatorname{ord}_{v}\left(\omega^{\prime}\right) \geq-2 \operatorname{ord}_{v}(\mathfrak{A})$ and $\omega^{\prime} \in L\left(\mathfrak{D}_{1}+2 \mathfrak{D}_{2}+2 \mathfrak{A}\right)$.

Proposition 3. For all divisors $\mathfrak{D}>0$ with $\operatorname{deg}\left(\mathfrak{D}_{2}\right) \geq 2 g_{K}-1$ we have $N(\mathfrak{D})=2 \phi(\mathfrak{D})$. If $\mathfrak{D}>0$ and $v_{0} \in M(K) \backslash \operatorname{Supp}(\mathfrak{D})$ with $\operatorname{deg}\left(v_{0}\right) \geq 2 g_{k}-1$, then $N\left(\mathfrak{D}+v_{0}\right)-2 \phi\left(\mathfrak{D}+v_{0}\right)=2 \phi(\mathfrak{D})-N(\mathfrak{D})$. If $v_{0}$ is any place with $\operatorname{deg}\left(v_{0}\right) \geq 2 g_{K}-1$, then $N\left(v_{0}\right)=2 \phi\left(v_{0}\right)$.

Proof. Suppose $\mathfrak{D}>0$. Choose a place $v_{0} \notin \operatorname{Supp}(\mathfrak{D})$ with $\operatorname{deg}\left(v_{0}\right) \geq 2 g_{K}-1$. By Lemma 6 , every $F \in S(\mathfrak{D}) \cup S\left(\mathfrak{D}+v_{0}\right)$ has a generator in

$$
\left\{\alpha \in L\left(\mathfrak{D}_{1}+2 \mathfrak{D}_{2}+2 n_{F} v_{0}\right): \operatorname{ord}_{v}(\alpha)=1-2 \operatorname{ord}_{v}(\mathfrak{D}) \text { for all } v \in \operatorname{Supp}(\mathfrak{D})\right\}
$$

for some integer $n_{F} \geq 0$. Since $S(\mathfrak{D}) \cup S\left(\mathfrak{D}+v_{0}\right)$ is a finite set, we may choose a positive integer $n$ such that all $F \in S(\mathfrak{D}) \cup S\left(\mathfrak{D}+v_{0}\right)$ have a generator in the set

$$
S=\left\{\omega \in L\left(\mathfrak{D}_{1}+2 \mathfrak{D}_{2}+2 n v_{0}\right): \operatorname{ord}_{v}(\omega)=1-2 \operatorname{ord}_{v}(\mathfrak{D}) \text { for all } v \in \operatorname{Supp}(\mathfrak{D})\right\}
$$

By Lemma 7 , if $F$ is any quadratic extension of $K$ with a generator in $S$, then $\operatorname{Disc}_{K}(F)=$ $2 \mathfrak{D}+2 j v_{0}$ for some $0 \leq j \leq n$. Conversely, every element $\omega \in S$ is a generator of a quadratic extension since $\operatorname{ord}_{v}(\omega)<0$ and odd for all $v \in \operatorname{Supp}(\mathfrak{D}) \neq \emptyset$. For all $j=0, \ldots, n$ set

$$
S_{j}=\left\{F \in S\left(\mathfrak{D}+j v_{0}\right): F \text { has a generator in } S\right\}
$$


and for all $j=1, \ldots, n$, set

$$
S_{j}^{\prime}=\left\{F \in S\left(\mathfrak{D}+j v_{0}\right): \quad F \text { has a generator in } L^{\prime}\left(\mathfrak{D}_{1}+v_{0}+2 \mathfrak{D}_{2}+(2 j-2) v_{0}\right)\right\}
$$

Note that $\left(\mathfrak{D}+j v_{0}\right)_{1}=\mathfrak{D}_{1}+v_{0}$ and $\left(\mathfrak{D}+j v_{0}\right)_{2}=\mathfrak{D}_{2}+(j-1) v_{0}$ for $j \geq 1$. Set $N_{j}=\# S_{j}$ for all $j=0, \ldots, n$ and $N_{j}^{\prime}=\# S_{j}^{\prime}$ for $j \geq 1$. Clearly $S_{j}^{\prime} \subseteq S_{j}$ for all $j=1, \ldots, n$. Also $S_{0}=S(\mathfrak{D})$ and $S_{1}=S\left(\mathfrak{D}+v_{0}\right)$ by construction, so that $N_{0}=N(\mathfrak{D}), N_{1}=N\left(\mathfrak{D}+v_{0}\right)$ and $N_{j}^{\prime} \leq N_{j}$ for $j \geq 1$.

Since $\operatorname{deg}\left(v_{0}\right) \geq 2 g_{K}-1$ and $n>0$ we have

$$
\# S=q^{2 n \operatorname{deg}\left(v_{0}\right)+1-g_{K}} \phi\left(\mathfrak{D}_{1}+2 \mathfrak{D}_{2}\right)=q^{2 n \operatorname{deg}\left(v_{0}\right)+\operatorname{deg}\left(\mathfrak{D}_{2}\right)+1-g_{K}} \phi(\mathfrak{D})
$$

by Lemma 4, so that by Lemma 7

$$
\begin{aligned}
\sum_{j=0}^{n} N_{i}=\frac{2 \# S}{q^{l\left(\mathfrak{D}_{2}+n v_{0}\right)}} & =\frac{2 q^{2 n \operatorname{deg}\left(v_{0}\right)+\operatorname{deg}\left(\mathfrak{D}_{2}\right)+1-g_{K}} \phi(\mathfrak{D})}{q^{n \operatorname{deg}\left(v_{0}\right)+\operatorname{deg}\left(\mathfrak{D}_{2}\right)+1-g_{K}}} \\
& =2 q^{n \operatorname{deg}\left(v_{0}\right)} \phi(\mathfrak{D}) .
\end{aligned}
$$

By Lemma 5 , for all $j=2, \ldots, n$ we have

$$
\begin{aligned}
N_{j}^{\prime} & =2 \phi\left(\mathfrak{D}+j v_{0}\right) \\
& =2 \phi(\mathfrak{D}) \phi\left(j v_{0}\right) \\
& =2 \phi(\mathfrak{D})\left(q^{j \operatorname{deg}\left(v_{0}\right)}-q^{(j-1) \operatorname{deg}\left(v_{0}\right)}\right) .
\end{aligned}
$$

Therefore

$$
\begin{aligned}
\sum_{j=0}^{n} N_{j} \geq N_{0}+N_{1}^{\prime}+\sum_{j=2}^{n} N_{j}^{\prime} & =N_{0}+N_{1}^{\prime}+\sum_{j=2}^{n} 2 \phi(\mathfrak{D})\left(q^{j \operatorname{deg}\left(v_{0}\right)}-q^{(j-1) \operatorname{deg}\left(v_{0}\right)}\right) \\
& =N_{0}+N_{1}^{\prime}+2 \phi(\mathfrak{D})\left(q^{n \operatorname{deg}\left(v_{0}\right)}-q^{\operatorname{deg}\left(v_{0}\right)}\right) .
\end{aligned}
$$

Suppose $\operatorname{deg}\left(\mathfrak{D}_{2}\right) \geq 2 g_{K}-1$. Then since $\left(\mathfrak{D}+v_{0}\right)_{2}=\mathfrak{D}_{2}$ we have $(6)$ for $j=1$ as well. Combining this with (5) and (7) yields

$$
\begin{aligned}
2 \phi(\mathfrak{D}) q^{n \operatorname{deg}\left(v_{0}\right)} & \geq N_{0}+2 \phi(\mathfrak{D})\left(q^{n \operatorname{deg}\left(v_{0}\right)}-1\right), \\
& 2 \phi(\mathfrak{D}) \geq N_{0} .
\end{aligned}
$$

But $N_{0}=N(\mathfrak{D})$ by construction and $N(\mathfrak{D}) \geq 2 \phi(\mathfrak{D})$ by Lemma 5 . Hence $N_{0}=N(\mathfrak{D})=2 \phi(\mathfrak{D})$.

Now suppose simply that $\mathfrak{D}>0$. Since $\operatorname{deg}\left(\mathfrak{D}_{2}+(j-1) v_{0}\right) \geq(j-1) \operatorname{deg}\left(v_{0}\right) \geq 2 g_{K}-1$ for all $j=2, \ldots, n$, by what we have already proven we have

$$
N_{j}^{\prime}=N_{j}=N\left(\mathfrak{D}+j v_{0}\right)=2 \phi\left(\mathfrak{D}+j v_{0}\right)=2 \phi(\mathfrak{D})\left(q^{j \operatorname{deg}\left(v_{0}\right)}-q^{(j-1) \operatorname{deg}\left(v_{0}\right)}\right)
$$


for all $j=2, \ldots, n$. Thus by (5),

$$
\begin{aligned}
2 q^{n \operatorname{deg}\left(v_{0}\right)} \phi(\mathfrak{D}) & =N_{0}+N_{1}+\sum_{j=2}^{n} N_{j} \\
& =N(\mathfrak{D})+N\left(\mathfrak{D}+v_{0}\right)+2 \phi(\mathfrak{D})\left(q^{n \operatorname{deg}\left(v_{0}\right)}-q^{\operatorname{deg}\left(v_{0}\right)}\right),
\end{aligned}
$$

so that

$$
\begin{aligned}
2 \phi(\mathfrak{D})-N(\mathfrak{D}) & =N\left(\mathfrak{D}+v_{0}\right)-2 \phi(\mathfrak{D}) q^{\operatorname{deg}\left(v_{0}\right)}+2 \phi(\mathfrak{D}) \\
& =N\left(\mathfrak{D}+v_{0}\right)-2 \phi\left(\mathfrak{D}+v_{0}\right) .
\end{aligned}
$$

Finally, if $\mathfrak{D}=0$ we have the same argument as above except that now not all elements of $S=L\left(2 n v_{0}\right)$ need generate a quadratic extension and we have one quadratic extension with a generator in $\mathbb{F}_{q}=L(0)$ (the field obtained from $K$ be extending the field of constants to $q^{2}$ ). This extension has $q^{l\left(n v_{0}\right)} / 2$ generators in $S$ by Lemma 7 . Any $\beta \in L\left(n v_{0}\right)$ gives a $\beta^{2}+\beta \in L\left(2 n v_{0}\right)$ and vice-versa. Once again, $\beta \mapsto \beta^{2}+\beta$ is a two-to-one map on $L\left(n v_{0}\right)$, so there are $q^{l\left(n v_{0}\right)} / 2$ elements of $S$ that do not generate a quadratic extension of $K$. Therefore (5) is replaced by

$$
2+\sum_{j=1}^{n} N_{j}=\frac{2 \# S}{q^{l\left(n v_{0}\right)}}=2 q^{l\left(2 n v_{0}\right)-l\left(n v_{0}\right)}=2 q^{n \operatorname{deg}\left(v_{0}\right)} .
$$

Using $N_{j}=2 \phi\left(j v_{0}\right)$ for $j \geq 2$ as above yields

$$
2+N\left(v_{0}\right)+2\left(q^{n \operatorname{deg}\left(v_{0}\right)}-q^{\operatorname{deg}\left(v_{0}\right)}\right)=2 q^{n \operatorname{deg}\left(v_{0}\right)},
$$

whence $N\left(v_{0}\right)=2 q^{\operatorname{deg}\left(v_{0}\right)}-2=2 \phi\left(v_{0}\right)$.

Corollary. If $g_{K}=0$, then $N(\mathfrak{D})=2 \phi(\mathfrak{D})$ for all $\mathfrak{D}>0$. If $g_{K}>0$, then $N(\mathfrak{D})=2 \phi(\mathfrak{D})$ if no place $v \in \operatorname{Supp}(\mathfrak{D})$ has $\operatorname{deg}(v)<2 g_{K}-1$ or if $\operatorname{deg}\left(\mathfrak{D}_{2}\right) \geq 2 g_{K}-1$. For all other divisors $\mathfrak{D}>0$ we have

$$
|N(\mathfrak{D})-2 \phi(\mathfrak{D})| \ll 1
$$

where the implicit constant depends only on $K$.

Proof. The case where $g_{K}=0$ follows immediately from Propositions 1 and 3 . Suppose $g_{K}>0$ and write

$$
\mathfrak{D}^{\prime}=\sum_{\substack{v \in \operatorname{Supp}(\mathfrak{D}) \\ \operatorname{ord}_{v}(\mathfrak{D})>1 \operatorname{or} \operatorname{deg}(v)<2 g_{K}-1}} \operatorname{ord}_{v}(\mathfrak{D}) \cdot v .
$$


Note that $\mathfrak{D}_{2}=\mathfrak{D}_{2}^{\prime}$ by construction. According to Proposition 3 ,

$$
|N(\mathfrak{D})-2 \phi(\mathfrak{D})|=\left|N\left(\mathfrak{D}^{\prime}\right)-2 \phi\left(\mathfrak{D}^{\prime}\right)\right| \text {. }
$$

Now if $\operatorname{deg}\left(\mathfrak{D}_{2}\right)=\operatorname{deg}\left(\mathfrak{D}_{2}^{\prime}\right) \geq 2 g_{K}-1$, then $N\left(\mathfrak{D}^{\prime}\right)=2 \phi\left(\mathfrak{D}^{\prime}\right)$ by Proposition 3 . If $\operatorname{deg}\left(\mathfrak{D}_{2}^{\prime}\right)<2 g_{K}-1$, then $\mathfrak{D}^{\prime}$ is an effective divisor with $\operatorname{deg}\left(\mathfrak{D}^{\prime}\right) \ll 1$. The Corollary follows in this case from $(0)$.

\section{Characters and Generators}

In this section we reinterpret $\chi(F / \mathfrak{C})$ in terms of generators and certain characters. We then look at certain character sums and prove a result akin to the Polya-Vinogradov inequality for incomplete multiplicative character sums.

As before we fix a function field $K$ and write $q_{K}=q$. When $F$ is a separable quadratic extension of $K$ and $\mathfrak{C} \in \operatorname{Div}(K)$ is an effective divisor, we desire a description of $\chi(F / \mathfrak{C})$ in terms of a generator of $F$. Recall that we say a (separable) quadratic extension $F$ of $K$ is generated by an $\omega \in K$ if $F$ is the splitting field of the polynomial $P_{\omega}(Y)$ given by

$$
P_{\omega}(Y)= \begin{cases}Y^{2}-\omega & \text { if } q \text { is odd, } \\ Y^{2}+Y+\omega & \text { if } q \text { is even. }\end{cases}
$$

For any place $v \in M(K)$, set $K_{v}$ to be the topological completion of $K$ at the place $v$ and set $\mathfrak{O}_{v}=\left\{\alpha_{v} \in K_{v}: \operatorname{ord}_{v}\left(\alpha_{v}\right) \geq 0\right\}$. Let $\pi_{v} \in \mathfrak{O}_{v}$ with $\operatorname{ord}_{v}\left(\pi_{v}\right)=1$. Then $\pi_{v} \mathfrak{O}_{v}$ is the unique maximal ideal in the discrete valuation ring $\mathfrak{O}_{v}$. The residue class field $\mathfrak{O}_{v} / \pi_{v} \mathfrak{O}_{v}$ is isomorphic to $\mathbb{F}_{q^{\operatorname{deg}(v)}}$ by definition/construction. Now for any $\alpha_{v} \in \mathfrak{O}_{v}$ define

$$
P_{\alpha_{v}}(Y)= \begin{cases}Y^{2}-\alpha_{v} & \text { if } q \text { is odd } \\ Y^{2}+Y+\alpha_{v} & \text { if } q \text { is even. }\end{cases}
$$

Define $\chi_{v}\left(\alpha_{v}\right)$ to be 0,1 or -1 , depending on whether $P_{\alpha_{v}}$ is a square, a product of two distinct linear factors, or is irreducible over $\mathfrak{O}_{v} / \pi_{v} \mathfrak{O}_{v}$, respectively.

Recall that we denote the adele ring by $K_{\mathbb{A}}$. For a non-zero effective divisor $\mathfrak{C} \in \operatorname{Div}(K)$ set

$$
\mathfrak{O}_{\mathbb{A}}(\mathfrak{C})=\left\{\left(\alpha_{v}\right) \in K_{\mathbb{A}}: \alpha_{v} \in \mathfrak{O}_{v} \text { for all places } v \in \operatorname{Supp}(\mathfrak{C})\right\}
$$

and

$$
\pi_{\mathbb{A}}(\mathfrak{C})=\left\{\left(\alpha_{v}\right) \in \mathfrak{O}_{\mathbb{A}}(\mathfrak{C}): \alpha_{v} \in \pi_{v}^{\text {ord }_{v}(\mathfrak{C})} \mathfrak{O}_{v} \text { for all } v \in \operatorname{Supp}(\mathfrak{C})\right\}
$$

Then $\chi_{v}\left(\alpha_{v}\right)$ is defined for all $v \in \operatorname{Supp}(\mathfrak{C})$ whenever the adele $\left(\alpha_{v}\right) \in \mathfrak{O}_{\mathbb{A}}(\mathfrak{C})$, and we set

$$
\chi_{\mathfrak{C}}\left(\left(\alpha_{v}\right)\right)=\prod_{v \in \operatorname{Supp}(\mathfrak{C})} \chi_{v}\left(\alpha_{v}\right)^{\operatorname{ord}_{v}(\mathfrak{C})}
$$


Proposition 4. Suppose $K$ is a function field, $\mathfrak{C} \in \operatorname{Div}(K)$ with $\mathfrak{C}>0$ and $F$ is a quadratic extension of $K$ satisfying $q_{F}=q_{K}$ and $\left(\mathfrak{C}, \operatorname{Disc}_{K}(F)\right)=0$. Suppose $F$ is generated by $\omega \in \mathfrak{O}_{\mathbb{A}}(\mathfrak{C})$. If $q_{K}$ is even, then $\chi(F / \mathfrak{C})=\chi_{\mathfrak{C}}(\omega)$. If $q_{K}$ is odd, then $\chi(F / \mathfrak{C})=\chi_{\mathfrak{C}}(\omega)$ if $\operatorname{ord}_{v}(\omega)=0$ for all $v \in \operatorname{Supp}(\mathfrak{C})$, i.e., if $\omega+\pi_{\mathbb{A}}\left(\mathfrak{C}_{1}\right)$ is a unit in the quotient ring $\mathfrak{O}_{\mathbb{A}}(\mathfrak{C}) / \pi_{\mathbb{A}}\left(\mathfrak{C}_{1}\right)$.

Proof. Suppose $F$ is a quadratic extension generated by $\omega \in \mathfrak{O}_{\mathbb{A}}(\mathfrak{C})$ and let $y \in F$ satisfy $y^{2}=\omega$ if $q$ is odd, or $y^{2}+y=\omega$ if $q$ is even. The formal derivative of $P_{\omega}$ satisfies

$$
P_{\omega}^{\prime}(y)= \begin{cases}2 y & \text { if } q_{K} \text { is odd } \\ 1 & \text { if } q_{K} \text { is even }\end{cases}
$$

If $v \in \operatorname{Supp}(\mathfrak{C})$, then no place $w \in M(F)$ lying above $v$ ramifies by Proposition 1 , so that $0=$ $\operatorname{ord}_{w}\left(P_{\omega}^{\prime}(y)\right)=\operatorname{ord}_{w}\left(\operatorname{Diff}_{K}(F)\right)$ and $\{1, y\}$ is an integral basis for $F$ over $K$ at the place $w$ by [11, Theorem III.5.10]. The proposition now follows from [11, Theorem III.3.7].

With this in hand, we now make some pertinent observations about $\chi_{\mathfrak{C}}$ and $\mathfrak{O}_{\mathbb{A}}(\mathfrak{C})$.

Lemma 8. Suppose $K$ is a function field and $\mathfrak{C} \in \operatorname{Div}(K)$ with $\mathfrak{C}>0$. Then

$$
\mathfrak{O}_{\mathbb{A}}(\mathfrak{C}) / \pi_{\mathbb{A}}(\mathfrak{C}) \cong \prod_{v \in \operatorname{Supp}(\mathfrak{C})} \mathfrak{O}_{v} / \pi_{v}^{\operatorname{ord}_{v}(\mathfrak{C})} \mathfrak{O}_{v}
$$

and $\operatorname{dim}_{\mathbb{F}_{q_{K}}}\left(\mathfrak{O}_{\mathbb{A}}(\mathfrak{C}) / \pi_{\mathbb{A}}(\mathfrak{C})\right)=\operatorname{deg}(\mathfrak{C})$. If $\mathfrak{A}$ is any divisor with $\operatorname{Supp}(\mathfrak{A}) \cap \operatorname{Supp}(\mathfrak{C})=\emptyset$, then $L(\mathfrak{A}) \subset \mathfrak{O}_{\mathbb{A}}(\mathfrak{C})$, and if $\operatorname{deg}(\mathfrak{A}) \geq \operatorname{deg}(\mathfrak{C})+2 g_{K}-1$, then the image of $L(\mathfrak{A})$ under the canonical map $\mathfrak{O}_{\mathbb{A}}(\mathfrak{C}) \mapsto \mathfrak{O}_{\mathbb{A}}(\mathfrak{C}) / \pi_{\mathbb{A}}(\mathfrak{C})$ is $\mathfrak{O}_{\mathbb{A}}(\mathfrak{C}) / \pi_{\mathbb{A}}(\mathfrak{C})$

Proof. The first statement is obvious and the second follows immediately since $\operatorname{dim}_{\mathbb{F}_{q_{K}}}\left(\mathfrak{O}_{v} / \pi_{v} \mathfrak{O}_{v}\right)=$ $\operatorname{deg}(v)$ by definition.

Let $\theta_{\mathfrak{C}}: \mathfrak{O}_{\mathbb{A}}(\mathfrak{C}) \rightarrow \mathfrak{O}_{\mathbb{A}}(\mathfrak{C}) / \pi_{\mathbb{A}}(\mathfrak{C})$ denote the canonical map. Suppose $\mathfrak{A}$ is a divisor with $\operatorname{Supp}(\mathfrak{A}) \cap$ $\operatorname{Supp}(\mathfrak{C})=\emptyset$. Then $L(\mathfrak{A}) \subset \mathfrak{O}_{\mathbb{A}}(\mathfrak{C})$ directly from the definitions and

$$
\begin{aligned}
L(\mathfrak{A}) \cap \operatorname{ker}\left(\theta_{\mathfrak{C}}\right) & =L(\mathfrak{A}) \cap \pi_{\mathbb{A}}(\mathfrak{C}) \\
& =\left\{\alpha \in L(\mathfrak{A}): \operatorname{ord}_{v}(\alpha) \geq \operatorname{ord}_{v}(\mathfrak{C}) \text { all } v \in \operatorname{Supp}(\mathfrak{C})\right\} \\
& =L(\mathfrak{A}-\mathfrak{C}) .
\end{aligned}
$$

Suppose further that $\operatorname{deg}(\mathfrak{A}) \geq \operatorname{deg}(\mathfrak{C})+2 g_{K}-1$. Then the dimension of the image $\theta_{\mathfrak{C}}(L(\mathfrak{A}))$ is $l(\mathfrak{A})-l(\mathfrak{A}-\mathfrak{C})=\operatorname{deg}(\mathfrak{C})$ by the Riemann-Roch Theorem. This completes the proof. 
Lemma 9. Suppose $K$ is a function field and $a \in \mathbb{F}_{q_{K}}$ such that $a \neq b^{2}$ if $q_{K}$ is odd or $a \neq b^{2}+b$ if $q_{K}$ is even, for all $b \in \mathbb{F}_{q_{K}}$. Then for all places $v \in M(K)$ we have

$$
\chi_{v}(a)= \begin{cases}1 & \text { if } \operatorname{deg}(v) \text { is even } \\ -1 & \text { if } \operatorname{deg}(v) \text { is odd }\end{cases}
$$

In particular, for any divisor $\mathfrak{C}>0$ we have $\chi_{\mathfrak{C}}(a)=(-1)^{\operatorname{deg}(\mathfrak{C})}$ and if $\operatorname{deg}(\mathfrak{C})$ is odd

$$
\sum_{\substack{[F: K]=2 \\ g_{F}=m, q_{F}=q_{K}}} \chi(F / \mathfrak{C})=0
$$

for all $m \geq 0$.

Proof. Set $q=q_{K}$. Let $v \in M(K)$ and choose a $\pi_{v} \in \mathfrak{O}_{v}$ as above. We have $\mathbb{F}_{q^{\operatorname{deg}(v)}} \cong \mathfrak{O}_{v} / \pi_{v} \mathfrak{O}_{v}$ and $\mathbb{F}_{q} \cap \pi_{v} \mathfrak{O}_{v}=\{0\}$ since $\operatorname{ord}_{v}(b)=0 \neq 1$ for all non-zero $b \in \mathbb{F}_{q}$. Now $\left\{b+\pi_{v} \mathfrak{O}_{v}: b \in \mathbb{F}_{q}\right\} \cong \mathbb{F}_{q}$ is a subfield of the residue class field $\mathfrak{O}_{v} / \pi_{v} \mathfrak{O}_{v}$. As such, $Y^{2}-b$ (if $q$ is odd) or $Y^{2}+Y+b$ (if $q$ is even) splits over the residue class field $\mathfrak{O}_{v} / \pi_{v} \mathfrak{O}_{v}$ for $b \in \mathbb{F}_{q}$ exactly when the residue class field contains the splitting field; this splitting field is (isomorphic to) $\mathbb{F}_{q^{2}}$ when $b=a$. On the other hand, this is possible exactly when $\mathbb{F}_{q^{2}}$ is (isomorphically) a subfield of $\mathbb{F}_{q^{\operatorname{deg}(v)}}$. Thus $\chi_{v}(a)=-1$ for all places $v \in M(K)$ of odd degree and $\chi_{v}(a)=1$ for all places $v \in M(K)$ of even degree.

Now suppose $F$ is a (separable) quadratic extension of $K$ with $q_{F}=q$ and say $F$ is generated by $\omega \in K$. Then aw generates a quadratic extension $F^{\prime}$ with $\operatorname{Disc}_{K}(F)=\operatorname{Disc}_{K}\left(F^{\prime}\right)$ in odd characteristic and $a+\omega$ generates an $F^{\prime}$ with $\operatorname{Disc}_{K}(F)=\operatorname{Disc}_{K}\left(F^{\prime}\right)$ in characteristic 2. We can’t have $F^{\prime}=F$ in either case, since then $q_{F}=q^{2}$. By what we have shown, if $\operatorname{deg}(\mathfrak{C})$ is odd, then $\chi\left(F^{\prime} / \mathfrak{C}\right)=-\chi(F / \mathfrak{C})$. Finally, $b^{2} \omega$ in odd characteristic and $\omega+b^{2}+b$ in characteristic 2 both generate the original quadratic extension $F$ for all $b \in \mathbb{F}_{q}$. This shows the final equation in Lemma 9.

We now turn to a more general look at characters. For our purposes here, a character on an abelian group $G$ will mean a group homomorphism from $G$ into $\mathbb{C}^{\times}$. If $H \subseteq G$ is any finite subgroup and $\chi$ is a character on $G$, then we have

$$
\sum_{h \in H} \chi(h)= \begin{cases}|H| & \text { if } H \subseteq \operatorname{ker}(\chi) \\ 0 & \text { otherwise }\end{cases}
$$


The proof is trivial. Let $h_{0} \in H$; then

$$
\sum_{h \in H} \chi(h)=\sum_{h \in H} \chi\left(h+h_{0}\right)=\chi\left(h_{0}\right) \sum_{h \in H} \chi(h) .
$$

Now suppose $R$ is a finite commutative ring with identity. A multiplicative character on $R$ is a function $\Phi: R \rightarrow \mathbb{C}$ such that the restriction to the multiplicative group of units $R^{\times}$is a group character and $\Phi=0$ on the complement. When the restriction to $R^{\times}$is the trivial character, we call $\Phi$ the principal character on $R$. We say the multiplicative character $\Phi$ is primitive if for all non-zero ideals $I \subseteq R$ there are units $u, v \in R^{\times}$with $u \equiv v \bmod I$ and $\Phi(u) \neq \Phi(v)$.

We readily see that $\chi_{\mathfrak{C}}$ is a character on $\mathfrak{O}_{\mathbb{A}}(\mathfrak{C})$ when $q_{K}$ is even, with $\operatorname{ker}\left(\chi_{\mathfrak{C}}\right) \supseteq \pi_{\mathbb{A}}\left(\mathfrak{C}_{1}\right)$. When $q_{K}$ is odd, we readily see that $\Phi_{\mathfrak{C}}\left(\left(\alpha_{v}\right)+\pi_{\mathbb{A}}\left(\mathfrak{C}_{1}\right)\right)=\chi_{\mathfrak{C}}\left(\left(\alpha_{v}\right)\right)$ defines a multiplicative character on the quotient ring $\mathfrak{O}_{\mathbb{A}}(\mathfrak{C}) / \pi_{\mathbb{A}}\left(\mathfrak{C}_{1}\right)$.

In what follows we will take the convolution of a multiplicative character $\Phi$ on a finite commutative ring with identity $R$ with a fixed character $\chi$ on the additive structure of $R$ :

$$
\tau(\Phi):=\sum_{r \in R} \Phi(r) \chi(r)
$$

We require the character $\chi$ to be non-trivial on all non-zero principal ideals; we assume this is the case in what follows. Thus, for all non-zero $h \in R$ we have

$$
\sum_{g \in R} \chi(g h)=0
$$

by (8).

Lemma 10. Suppose $R$ is a finite commutative ring with identity and $\Phi$ is a multiplicative character on $R$. Then for all $u \in R^{\times}$we have

$$
\sum_{r \in R} \overline{\Phi(r)} \chi(u r)=\Phi(u) \tau(\bar{\Phi})
$$

where the overline denotes complex conjugation.

Proof. Setting $r^{\prime}=u r$ below, we have

$$
\begin{aligned}
\Phi(u) \sum_{r \in R} \Phi(r) \chi(u r) & =\sum_{r \in R} \Phi(u r) \chi(u r) \\
& =\sum_{r^{\prime} \in R} \Phi\left(r^{\prime}\right) \chi\left(r^{\prime}\right) \\
& =\tau(\Phi) .
\end{aligned}
$$

The lemma follows upon replacing $\Phi$ above by $\bar{\Phi}$ and noting that $\overline{\Phi(u)}=(\Phi(u))^{-1}$. 
Lemma 11. Suppose $R$ is a finite commutative ring with identity and $\Phi$ is a primitive multiplicative character on $R$. Suppose $I$ is a non-zero ideal of $R$ and $r_{0} \in R$. Then

$$
\sum_{\substack{r \in R \\ r \equiv r_{0} \bmod I}} \Phi(r)=0 .
$$

Proof. Let $u, v \in R^{\times}$with $u \equiv v \bmod I$ and $\Phi(u) \neq \Phi(v)$. Then $w=u^{-1} v$ satisfies $\Phi(w) \neq 1$ and $w \equiv 1 \bmod I$. We thus have (with $r^{\prime}=r w^{-1}$ )

$$
\begin{aligned}
\sum_{\substack{r \in R \\
r \equiv r_{0} \bmod I}} \Phi(r)= & \sum_{\substack{r \in R \\
r \equiv w r_{0} \bmod I}} \Phi(r) \\
= & \sum_{\substack{r \in R \\
r w^{-1} \equiv r_{0} \bmod I}} \Phi(r) \\
= & \sum_{\substack{r^{\prime} \in R \\
r^{\prime} \equiv r_{0} \bmod I}} \Phi\left(r^{\prime} w\right) \\
= & \Phi(w) \sum_{\substack{r^{\prime} \in R \\
r^{\prime} \equiv r_{0} \bmod I}} \Phi\left(r^{\prime}\right) .
\end{aligned}
$$

The lemma follows.

Lemma 12. Suppose $R$ is a finite commutative ring with identity and $\Phi$ is a primitive multiplicative character on $R$. Then for all $r_{0} \in R$ we have

$$
\sum_{r \in R} \overline{\Phi(r)} \chi\left(r r_{0}\right)=\Phi\left(r_{0}\right) \tau(\bar{\Phi})
$$

Also, $|\tau(\Phi)|^{2}=\# R$.

Proof. The first part is simply Lemma 10 when $r_{0}$ is a unit, so assume otherwise (but $r_{0} \neq 0$ ) and let $I=\operatorname{ann}\left(r_{0}\right) \neq\{0\}$. Write $R / I=\left\{r_{j}+I: j=1, \ldots, n\right\}$. Then by Lemma 11

$$
\begin{aligned}
\sum_{r \in R} \Phi(r) \overline{\chi\left(r r_{0}\right)} & =\sum_{j=1}^{n} \sum_{\substack{r \in R \\
r \equiv r_{j} \bmod I}} \Phi(r) \overline{\chi\left(r r_{0}\right)} \\
& =\sum_{j=1}^{n} \overline{\chi\left(r_{j} r_{0}\right)} \sum_{\substack{r \in R \\
r \equiv r_{j} \bmod I}} \Phi(r) \\
& =0 .
\end{aligned}
$$


Since $\Phi\left(r_{0}\right)=0$ by hypothesis, the first part of the lemma follows when $r_{0} \neq 0$. When $r_{0}=0$ we have

$$
\sum_{r \in R} \overline{\Phi(r)} \chi(r 0)=\overline{\sum_{r \in R} \Phi(r)}=0=\Phi(0) \tau(\bar{\Phi})
$$

by (8).

Now since $\operatorname{ker}(\chi)$ doesn't contain any non-zero principal ideal

$$
\begin{aligned}
\# R^{\times}|\tau(\Phi)|^{2} & =\sum_{r \in R}\left|\sum_{s \in R} \Phi(s) \chi(r s)\right|^{2} \\
& =\sum_{r \in R}\left(\sum_{s \in R} \Phi(s) \chi(r s)\right)\left(\sum_{t \in R} \overline{\Phi(t) \chi(r t)}\right) \\
& =\sum_{r \in R}\left(\sum_{s \in R} \Phi(s) \chi(r s)\right)\left(\sum_{t \in R} \overline{\Phi(t)} \chi(-r t)\right) \\
& =\sum_{s \in R} \sum_{t \in R} \Phi(s) \overline{\Phi(t)} \sum_{r \in R} \chi((s-t) r) \\
& =\sum_{s \in R} \Phi(s) \overline{\Phi(s)} \# R \\
& =\sum_{s \in R}|\Phi(s)|^{2} \# R \\
& =\# R^{\times} \# R .
\end{aligned}
$$

Lemma 13. Suppose $R$ is a finite commutative ring with identity and $\Phi$ is a primitive character on $R$. Then for all subgroups $H \subseteq R$ of the additive structure of $R$, we have

$$
\left|\sum_{h \in H} \Phi(h)\right| \leq \frac{\#\left\{u \in R^{\times}: u H \subseteq \operatorname{ker}(\chi)\right\} \cdot \# H}{\sqrt{\# R}} .
$$

Proof. By Lemma 12

$$
\Phi(h)=\frac{1}{\tau(\bar{\Phi})} \sum_{r \in R} \overline{\Phi(r)} \chi(r h)
$$

for all $h \in H$ and $|\tau(\bar{\Phi})|=\sqrt{\# R}$. Thus

$$
\begin{aligned}
\sqrt{\# R}\left|\sum_{h \in H} \Phi(h)\right| & =\left|\sum_{h \in H} \sum_{r \in R} \overline{\Phi(r)} \chi(r h)\right| \\
& \leq \sum_{r \in R}|\overline{\Phi(r)}|\left|\sum_{h \in H} \chi(r h)\right| \\
& =\sum_{u \in R^{\times}}\left|\sum_{h \in H} \chi(u h)\right| .
\end{aligned}
$$


By (8), the inner sum here is zero unless $u H \subseteq \operatorname{ker}(\chi)$, and if that is the case, then the inner sum is simply $\# H$.

We now return to the more specific situation where our ring is $\mathfrak{O}_{\mathbb{A}}(\mathfrak{C}) / \pi_{\mathbb{A}}(\mathfrak{C})$. Our first goal is an estimate for what will play the role of the subgroup $H$ in Lemma 13.

Lemma 14. Let $K$ be a function field and suppose $\mathfrak{C} \in \operatorname{Div}(K)$ with $\mathfrak{C}>0$. Denote the canonical map $\mathfrak{O}_{\mathbb{A}}(\mathfrak{C}) \rightarrow \mathfrak{O}_{\mathbb{A}}(\mathfrak{C}) / \pi_{\mathbb{A}}(\mathfrak{C})$ by $\theta_{\mathfrak{C}}$. Suppose $\mathfrak{D} \in \operatorname{Div}(K)$ is an effective divisor with $(\mathfrak{C}, \mathfrak{D})=0$ and $v_{0} \in M(K)$ with $v_{0} \notin \operatorname{Supp}(\mathfrak{C}) \cup \operatorname{Supp}(\mathfrak{D})$. Then for all integers $n$ and all proper subgroups $G$ of the additive structure of $\mathfrak{O}_{\mathbb{A}}(\mathfrak{C}) / \pi_{\mathbb{A}}(\mathfrak{C})$ we have

$$
\#\left\{u \in\left(\mathfrak{O}_{\mathbb{A}}(\mathfrak{C}) / \pi_{\mathbb{A}}(\mathfrak{C})\right)^{\times}: u \theta_{\mathfrak{C}}\left(L\left(n v_{0}-\mathfrak{D}\right)\right) \subseteq G\right\} \ll q_{K}^{\operatorname{deg}(\mathfrak{C})+\operatorname{deg}(\mathfrak{D})+(1-n) \operatorname{deg}\left(v_{0}\right)}
$$

where the implicit constant depends only on $K$.

Proof. For notational convenience set $R=\mathfrak{O}_{\mathbb{A}}(\mathfrak{C}) / \pi_{\mathbb{A}}(\mathfrak{C})$ and set $q=q_{K}$. If $\left\{u \in R^{\times}: u \theta_{\mathfrak{C}}\left(L\left(n v_{0}-\right.\right.\right.$ $\mathfrak{D})) \subseteq G$ is not empty, then by replacing $G$ with $u^{-1} G$ for some $u \in R^{\times}$, we see that we may assume $\theta_{\mathfrak{C}}\left(L\left(n v_{0}-\mathfrak{D}\right)\right) \subseteq G$. Set

$$
\begin{aligned}
& n_{0}=\min \left\{j \in \mathbb{Z}: j \operatorname{deg}\left(v_{0}\right)-\operatorname{deg}(\mathfrak{D}) \geq 2 g_{K}-1\right\}, \\
& n_{1}=\max \left\{j \in \mathbb{Z}: \theta_{\mathfrak{C}}\left(L\left(j v_{0}-\mathfrak{D}\right)\right) \subseteq G\right\},
\end{aligned}
$$

and $n_{2} \in \mathbb{Z}$ to be the unique solution to

$$
\operatorname{deg}(\mathfrak{C})+2 g_{K}-1 \leq n_{2} \operatorname{deg}\left(v_{0}\right)<\operatorname{deg}(\mathfrak{C})+2 g_{K}-1+\operatorname{deg}\left(v_{0}\right)
$$

Then $n \leq n_{1}$ by our hypothesis above. Since $\# R=q^{\operatorname{deg}(\mathfrak{C})}$ by Lemma 8 , we see that we may assume $n \geq n_{0}$. Also, as in the proof of Lemma 8 we see that both $\theta_{\mathfrak{C}}\left(L\left(n_{2} v_{0}\right)\right)$ and $\theta_{\mathfrak{C}}\left(L\left(\left(n_{2}+n_{0}\right) v_{0}\right)\right)$ are $R$; the last equality implies that $n_{2}+n_{0} \geq n_{1}$. In summary, we have $\theta_{\mathfrak{C}}\left(L\left(n_{2} v_{0}\right)\right)=R$ and we may assume that $n_{0} \leq n \leq n_{1} \leq n_{2}+n_{0}$.

Set

$$
G_{n}=\left\{r \in R: r \theta_{\mathfrak{C}}\left(L\left(n v_{0}-\mathfrak{D}\right)\right) \subseteq G\right\}
$$

Clearly $G_{n}$ is a subgroup of the additive structure of $R$. We will estimate the order of $G_{n}$.

Suppose $\alpha \in L\left(\left(n_{1}-n\right) v_{0}\right)$ and $\beta \in L\left(n v_{0}-\mathfrak{D}\right)$. Then $\alpha \beta \in L\left(n_{1} v_{0}-\mathfrak{D}\right)$ so that $\theta_{\mathfrak{C}}(\alpha \beta) \in G$ by the definition of $n_{1}$, whence $\alpha \in G_{n}$ and $G_{n} \supseteq \theta_{\mathfrak{C}}\left(L\left(\left(n_{1}-n\right) v_{0}\right)\right)$. 
By definition there is an $\alpha_{0} \in L\left(\left(n_{1}+1\right) v_{0}-\mathfrak{D}\right)$ with $\theta_{\mathfrak{C}}\left(\alpha_{0}\right) \notin G$. Suppose $\alpha \in L\left(\left(n_{1}-n_{0}\right) v_{0}\right)$ and write $\operatorname{ord}_{v_{0}}(\alpha)=j-n_{1}$ for some $j \geq n_{0}$. By the definition of $n_{0}$ we have $j \operatorname{deg}\left(v_{0}\right)-\operatorname{deg}(\mathfrak{D}) \geq$ $2 g_{K}-1$. Thus, by the Riemann-Roch Theorem

$$
\begin{aligned}
\operatorname{dim}_{\mathbb{F}_{q}}\left(L\left((j+1) v_{0}-\mathfrak{D}\right) / L\left(j v_{0}-\mathfrak{D}\right)\right)= & l\left((j+1) v_{0}-\mathfrak{D}\right)-l\left(j v_{0}-\mathfrak{D}\right) \\
= & (j+1) \operatorname{deg}\left(v_{0}\right)-\operatorname{deg}(\mathfrak{D})+1-g_{K} \\
& -\left(j \operatorname{deg}\left(v_{0}\right)-\operatorname{deg}(\mathfrak{D})+1-g_{K}\right) \\
= & \operatorname{deg}\left(v_{0}\right) .
\end{aligned}
$$

Similarly,

$$
\operatorname{dim}_{\mathbb{F}_{q}}\left(L\left(\left(n_{1}+1\right) v_{0}-\mathfrak{D}\right) / L\left(n_{1} v_{0}-\mathfrak{D}\right)\right)=\operatorname{deg}\left(v_{0}\right) .
$$

Let $\beta_{1}, \ldots, \beta_{\operatorname{deg}\left(v_{0}\right)} \in L\left((j+1) v_{0}-\mathfrak{D}\right)$ be linearly independent over $L\left(j v_{0}-\mathfrak{D}\right)$. Then we see that $\alpha \beta_{1}, \ldots, \alpha \beta_{\operatorname{deg}\left(v_{0}\right)} \in L\left(\left(n_{1}+1\right) v_{0}-\mathfrak{D}\right)$ are linearly independent over $L\left(n_{1} v_{0}-\mathfrak{D}\right)$. Thus, there is a $\beta \in L\left((j+1) v_{0}-\mathfrak{D}\right)$ with $\alpha \beta \equiv \alpha_{0} \bmod L\left(n_{1} v_{0}-\mathfrak{D}\right)$. Since $\theta_{\mathfrak{C}}\left(\alpha_{0}\right) \notin G$ and $\theta_{\mathfrak{C}}\left(L\left(n_{1} v_{0}-\mathfrak{D}\right)\right) \subseteq G$ by hypotheses, we see that $\theta_{\mathfrak{C}}(\alpha) \notin G_{n}$ whenever $j+1 \leq n$. In other words, if $\alpha \in L\left(\left(n_{1}-n_{0}\right) v_{0}\right)$ and $\theta_{\mathfrak{C}}(\alpha) \in G_{n}$, then $\alpha \in L\left(\left(n_{1}-n\right) v_{0}\right)$.

Write $q=p^{r}$ where the prime $p$ is the characteristic of $K$. All of our groups and subgroups above are vector spaces over $\mathbb{F}_{p}$. Since $n_{2} \geq n_{1}-n_{0}$, we have by the Riemann-Roch Theorem and Clifford's Theorem

$$
\begin{aligned}
\operatorname{dim}_{\mathbb{F}_{q}}\left(L\left(n_{2} v_{0}\right) / L\left(\left(n_{1}-n_{0}\right) v_{0}\right)\right) & =l\left(n_{2} v_{0}\right)-l\left(\left(n_{1}-n_{0}\right) v_{0}\right) \\
& \leq\left(n_{2}-n_{1}+n_{0}\right) \operatorname{deg}\left(v_{0}\right)+l\left(\mathfrak{W}_{K}-\left(n_{1}-n_{0}\right) v_{0}\right) \\
& \leq\left(n_{2}-n_{1}+n_{0}\right) \operatorname{deg}\left(v_{0}\right)+g_{K},
\end{aligned}
$$

where $\mathfrak{W}_{K} \in \operatorname{Div}(K)$ is in the canonical class, so that

$$
\operatorname{dim}_{\mathbb{F}_{p}}\left(L\left(n_{2} v_{0}\right) / L\left(\left(n_{1}-n_{0}\right) v_{0}\right)\right) \leq r\left(n_{2}-n_{1}+n_{0}\right) \operatorname{deg}\left(v_{0}\right)+r g_{K}
$$

Set $d=r\left(n_{2}-n_{1}+n_{0}\right) \operatorname{deg}\left(v_{0}\right)+r g_{K}+1$ and let $r_{1}, \ldots, r_{d} \in G_{n}$. Since $\theta_{\mathfrak{C}}\left(L\left(n_{2} v_{0}\right)\right)=R \supseteq G_{n}$, there are $\alpha_{1}, \ldots, \alpha_{d} \in L\left(n_{2} v_{0}\right)$ with $\theta_{\mathfrak{C}}\left(\alpha_{i}\right)=r_{i}$ for $i=1, \ldots, d$.

Now there are $a_{1}, \ldots, a_{d} \in \mathbb{F}_{p}$, not all zero, such that $\alpha=\sum_{i=1}^{d} a_{i} \alpha_{i} \in L\left(\left(n_{1}-n_{0}\right) v_{0}\right)$ and $\theta_{\mathfrak{C}}(\alpha) \in G_{n}$. By what we proved above, $\alpha \in L\left(\left(n_{1}-n\right) v_{0}\right)$. Therefore

$$
\operatorname{dim}_{\mathbb{F}_{p}}\left(G_{n} / \theta_{\mathfrak{C}}\left(L\left(\left(n_{1}-n\right) v_{0}\right)\right)\right) \leq d-1
$$


But by the Riemann-Roch Theorem and Clifford's Theorem

$$
\begin{aligned}
\operatorname{dim}_{\mathbb{F}_{q}}\left(L\left(\left(n_{1}-n\right) v_{0}\right)\right) & =\left(n_{1}-n\right) \operatorname{deg}\left(v_{0}\right)+1-g_{K}+l\left(\mathfrak{W}_{K}-\left(n_{1}-n\right) v_{0}\right) \\
& \leq\left(n_{1}-n\right) \operatorname{deg}\left(v_{0}\right)+1,
\end{aligned}
$$

so that

$$
\begin{aligned}
\operatorname{dim}_{\mathbb{F}_{p}}\left(G_{n}\right) & <d+r\left(n_{1}-n\right) \operatorname{deg}\left(v_{0}\right)+r \\
& =r\left(n_{2}+n_{0}-n\right) \operatorname{deg}\left(v_{0}\right)+r\left(1+g_{K}\right)
\end{aligned}
$$

and

$$
\begin{aligned}
\# G_{n} & \ll q^{\left(n_{2}+n_{0}-n\right) \operatorname{deg}\left(v_{0}\right)} \\
& \ll q^{\operatorname{deg}(\mathfrak{C})+\operatorname{deg}(\mathfrak{D})+(1-n) \operatorname{deg}\left(v_{0}\right)} .
\end{aligned}
$$

We are now ready to state and prove our analog of the classical Polya-Vinogradov inequality.

Theorem 4. Suppose $K$ is a function field and $\mathfrak{C} \in \operatorname{Div}(K)$ satisfies $\mathfrak{C}>0$. Let $\Phi$ be a nonprincipal multiplicative character on the quotient ring $\mathfrak{O}_{\mathbb{A}}(\mathfrak{C}) / \pi_{\mathbb{A}}(\mathfrak{C})$, $\mathfrak{D}$ be an effective divisor with $(\mathfrak{C}, \mathfrak{D})=0$, and $v_{0} \in M(K)$ with $v_{0} \notin \operatorname{Supp}(\mathfrak{C}) \cup \operatorname{Supp}(\mathfrak{D})$. Let $\theta_{\mathfrak{C}}: \mathfrak{O}_{\mathbb{A}}(\mathfrak{C}) \rightarrow \mathfrak{O}_{\mathbb{A}}(\mathfrak{C}) / \pi_{\mathbb{A}}(\mathfrak{C})$ denote the canonical map. Then for all integers $n$ with $n \operatorname{deg}\left(v_{0}\right) \geq \operatorname{deg}(\mathfrak{C})+\operatorname{deg}(\mathfrak{D})+2 g_{K}-1$

$$
\sum_{r \in \theta_{\mathfrak{C}}\left(L\left(n v_{0}-\mathfrak{D}\right)\right)} \Phi(r)=0
$$

and for all other integers $n \geq 0$

$$
\left|\sum_{r \in \theta_{\mathfrak{C}}\left(L\left(n v_{0}-\mathfrak{D}\right)\right)} \Phi(r)\right| \ll \min \left\{q_{K}^{n \operatorname{deg}\left(v_{0}\right)-\operatorname{deg}(\mathfrak{D})}, q_{K}^{\operatorname{deg}(\mathfrak{C}) / 2+\operatorname{deg}\left(v_{0}\right)}\right\},
$$

where the implicit constant depends only on $K$.

To see the connection with the classical case, replace the function field $K$ with the field $\mathbb{Q}$ and set $v_{0}$ to be the place of $\mathbb{Q}$ corresponding to the usual Euclidean absolute value. Then $L\left(n v_{0}\right)$ corresponds to the set of integers of absolute value no greater than $n$, and the quotient ring $\mathfrak{O}_{\mathbb{A}}(\mathfrak{C}) / \pi_{\mathbb{A}}(\mathfrak{C})$ to $\mathbb{Z} / m \mathbb{Z}$ for some integer $m>1$.

Proof. Set $R=\mathfrak{O}_{\mathbb{A}}(\mathfrak{C}) / \pi_{\mathbb{A}}(\mathfrak{C})$ and $q_{K}=q$ as above. Whenever $n \operatorname{deg}\left(v_{0}\right) \geq \operatorname{deg}(\mathfrak{C})+\operatorname{deg}(\mathfrak{D})+2 g_{K}-1$ we have $\theta_{\mathfrak{C}}\left(L\left(n v_{0}-\mathfrak{D}\right)\right)=R$ as remarked above in the proof of Lemma 8 , so that this case is simply an application of (8). We also note that the bound $q^{n \operatorname{deg}\left(v_{0}\right)-\operatorname{deg}(\mathfrak{D})}$ is the trivial bound. 
The ideals of $R$ are of the form $\theta_{\mathfrak{C}}\left(\pi_{\mathbb{A}}(\mathfrak{A})\right)$ for effective divisors $\mathfrak{A} \leq \mathfrak{C}$. Let $\mathfrak{A}$ be minimal among such effective divisors with $\Phi(u)=\Phi(v)$ for all units $u, v \in R^{\times}$with $u \equiv v \bmod \theta\left(\pi_{\mathbb{A}}(\mathfrak{A})\right)$. (This minimal $\mathfrak{A} \neq 0$ since $\Phi$ is not the principal character, and $\mathfrak{A}=\mathfrak{C}$ when $\Phi$ is primitive.) Then $\mathfrak{O}_{\mathbb{A}}(\mathfrak{A}) \supseteq \mathfrak{O}_{\mathbb{A}}(\mathfrak{C})$, and if we denote by $\theta_{\mathfrak{A}}$ the canonical map $\theta_{\mathfrak{A}}: \mathfrak{O}_{\mathbb{A}}(\mathfrak{A}) \rightarrow \mathfrak{O}_{\mathbb{A}}(\mathfrak{A}) / \pi_{\mathbb{A}}(\mathfrak{A})$, then we see that there is a primitive character $\Phi^{*}$ on $\mathfrak{O}_{\mathbb{A}}(\mathfrak{A}) / \pi_{\mathbb{A}}(\mathfrak{A})$ with

$$
\Phi \circ \theta_{\mathfrak{C}}\left(\left(\alpha_{v}\right)\right)= \begin{cases}\Phi^{*} \circ \theta_{\mathfrak{A}}\left(\left(\alpha_{v}\right)\right) & \text { if } \operatorname{ord}_{v}\left(\alpha_{v}\right)=0 \text { for all } v \in \operatorname{Supp}(\mathfrak{C}) \backslash \operatorname{Supp}(\mathfrak{A}) \\ 0 & \text { otherwise }\end{cases}
$$

Set $\mathfrak{B}=\sum_{v \in \operatorname{Supp}(\mathfrak{C}) \backslash \operatorname{Supp}(\mathfrak{A})} v$. Then

$$
\begin{aligned}
& \left|\sum_{r \in \theta_{\mathfrak{C}}\left(L\left(n v_{0}-\mathfrak{D}\right)\right)} \Phi(r)\right| \leq\left|\sum_{\alpha \in L\left(n v_{0}-\mathfrak{D}\right)} \Phi \circ \theta_{\mathfrak{C}}(\alpha)\right| \\
& =\left|\sum_{\substack{\alpha \in L\left(n v_{0}-\mathfrak{D}\right) \\
\operatorname{ord}_{v}(\alpha)=0 \text { all } v \in \operatorname{Supp}(\mathfrak{B})}} \Phi^{*} \circ \theta_{\mathfrak{A}}(\alpha)\right| \\
& =\left|\sum_{\alpha \in L\left(n v_{0}-\mathfrak{D}\right)} \Phi^{*} \circ \theta_{\mathfrak{A}}(\alpha) \sum_{\substack{0 \leq \mathfrak{E} \leq \mathfrak{B} \\
\operatorname{ord}_{v}(\alpha) \geq \operatorname{ord}_{v}(\mathfrak{E}) \text { all } v \in \operatorname{Supp}(\mathfrak{B})}} \mu(\mathfrak{E})\right| \\
& =\left|\sum_{0 \leq \mathfrak{E} \leq \mathfrak{B}} \mu(\mathfrak{E}) \sum_{\alpha \in L\left(n v_{0}-\mathfrak{D}-\mathfrak{E}\right)} \Phi^{*} \circ \theta_{\mathfrak{A}}(\alpha)\right| .
\end{aligned}
$$

We note that there exists a character $\chi$ on $R$ with the required properties above (see [13, Chapter IV], for instance). Let $\mathfrak{E}$ be a divisor satisfying $0 \leq \mathfrak{E} \leq \mathfrak{B}$. Then we may apply Lemma 14 to get

$$
\#\left\{r \in\left(\mathfrak{O}_{\mathbb{A}}(\mathfrak{A}) / \pi_{\mathbb{A}}(\mathfrak{A})\right)^{\times}: r \theta_{\mathfrak{C}}\left(L\left(n v_{0}-\mathfrak{D}-\mathfrak{E}\right)\right) \subseteq \operatorname{ker}(\chi)\right\} \ll q^{\operatorname{deg}(\mathfrak{A})+\operatorname{deg}(\mathfrak{D}+\mathfrak{E})+(1-n) \operatorname{deg}\left(v_{0}\right)}
$$

Using this together with Lemma 8, Lemma 13 and the Riemann-Roch Theorem yields

$$
\begin{aligned}
\left|\sum_{r \in \theta_{\mathfrak{C}}\left(L\left(n v_{0}-\mathfrak{D}-\mathfrak{E}\right)\right)} \Phi^{*}(r)\right| & \leq \frac{q^{\operatorname{deg}(\mathfrak{A})+\operatorname{deg}(\mathfrak{D}+\mathfrak{E})+(1-n) \operatorname{deg}\left(v_{0}\right)} \cdot \# \theta_{\mathfrak{C}}\left(L\left(n v_{0}-\mathfrak{D}-\mathfrak{E}\right)\right)}{\sqrt{\# \mathfrak{O}_{\mathbb{A}}(\mathfrak{A}) / \pi_{\mathbb{A}}(\mathfrak{A})}} \\
& \ll \frac{q^{\operatorname{deg}(\mathfrak{A})+\operatorname{deg}(\mathfrak{D}+\mathfrak{E})+(1-n) \operatorname{deg}\left(v_{0}\right)} \cdot q^{l\left(n v_{0}-\mathfrak{D}-\mathfrak{E}\right)}}{\sqrt{q^{\operatorname{deg}(\mathfrak{A})}}} \\
& \ll \frac{q^{\operatorname{deg}(\mathfrak{A})+\operatorname{deg}(\mathfrak{D}+\mathfrak{E})+(1-n) \operatorname{deg}\left(v_{0}\right)} \cdot q^{n \operatorname{deg}\left(v_{0}\right)-\operatorname{deg}(\mathfrak{D}+\mathfrak{E})}}{\sqrt{q^{\operatorname{deg}(\mathfrak{A})}}} \\
& =q^{\operatorname{deg}(\mathfrak{A}) / 2+\operatorname{deg}\left(v_{0}\right)} .
\end{aligned}
$$


Also, as in the proof of Lemma $8 L\left(n v_{0}-\mathfrak{D}-\mathfrak{E}\right) \cap \operatorname{ker}\left(\theta_{\mathfrak{A}}\right)=L\left(n v_{0}-\mathfrak{D}-\mathfrak{E}-\mathfrak{A}\right)$. If $n \operatorname{deg}\left(v_{0}\right) \geq$ $\operatorname{deg}(\mathfrak{A})+\operatorname{deg}(\mathfrak{D}+\mathfrak{E})+2 g_{K}-1$, we have $\theta_{\mathfrak{A}}\left(L\left(n v_{0}-\mathfrak{D}-\mathfrak{E}\right)\right)=\mathfrak{O}_{\mathbb{A}}(\mathfrak{A}) / \pi_{\mathbb{A}}(\mathfrak{A})$ and

$$
\sum_{r \in \theta_{\mathfrak{A}}\left(L\left(n v_{0}-\mathfrak{D}-\mathfrak{E}\right)\right)} \Phi^{*}(r)=0
$$

by (8). Hence we may assume that $\operatorname{deg}\left(n v_{0}-\mathfrak{D}-\mathfrak{E}-\mathfrak{A}\right)<2 g_{K}-1$, so that $\# L\left(n v_{0}-\mathfrak{D}-\mathfrak{E}-\mathfrak{A}\right) \ll 1$ and

$$
\left|\sum_{\alpha \in L\left(n v_{0}-\mathfrak{D}-\mathfrak{E}\right)} \Phi^{*} \circ \theta_{\mathfrak{A}}(\alpha)\right| \ll\left|\sum_{r \in \theta_{\mathfrak{A}}\left(L\left(n v_{0}-\mathfrak{D}-\mathfrak{E}\right)\right)} \Phi^{*}(r)\right| .
$$

We combine this with (9), (10) and Lemma 0 (setting $\epsilon=1 / 2$ there) to get

$$
\begin{aligned}
\sum_{r \in \theta_{\mathfrak{C}}\left(L\left(n v_{0}-\mathfrak{D}\right)\right)} \Phi(r) \mid & \leq \sum_{0 \leq \mathfrak{E} \leq \mathfrak{B}}\left|\sum_{\alpha \in L\left(n v_{0}-\mathfrak{D}-\mathfrak{E}\right)} \Phi^{*} \circ \theta_{\mathfrak{A}}(\alpha)\right| \\
& \ll q^{\operatorname{deg}(\mathfrak{B}) / 2} q^{\operatorname{deg}(\mathfrak{A}) / 2+\operatorname{deg}\left(v_{0}\right)} \\
& \leq q^{\operatorname{deg}(\mathfrak{C}) / 2+\operatorname{deg}\left(v_{0}\right)} .
\end{aligned}
$$

\section{The Main Estimates}

In this section we state and prove our three main estimates. These are the main tools with which we can prove results about sums involving $L$-functions.

Proposition 5. Suppose $K$ is a function field with $q_{K}=q$ odd and let $\mathfrak{C} \in \operatorname{Div}(K)$ be an effective divisor with $\mathfrak{C} \notin 2 \operatorname{Div}(K)$. Then for all positive integers $m$ and all $\epsilon>0$ we have

$$
\left|\sum_{\substack{[F: K]=2 \\ g F=m, q_{F}=q}} \chi(F / \mathfrak{C})\right| \ll q^{m} q^{(\epsilon+1 / 4) \operatorname{deg}(\mathfrak{C})}
$$

where the implicit constant depends only on $K$ and $\epsilon$. If $m \leq \operatorname{deg}(\mathfrak{C}) / 4$, then

$$
\left|\sum_{\substack{[F: K]=2 \\ g_{F}=m, q_{F}=q}} \chi(F / \mathfrak{C})\right| \ll q^{2 m},
$$

where the implicit constant depends only on $K$. 
Proposition 6. Suppose $K$ is a function field with $q_{K}=q$ even and let $\mathfrak{C} \in \operatorname{Div}(K)$ be an effective divisor with $\mathfrak{C} \notin 2 \operatorname{Div}(K)$. Then for all positive integers $m$ and all $\epsilon>0$ we have

$$
\left|\sum_{\substack{[F: K]=2 \\ g F=m, q_{F}=q}} \chi(F / \mathfrak{C})\right| \ll q^{m} \cdot q^{\epsilon \operatorname{deg}(\mathfrak{C})},
$$

where the implicit constant depends only on $K$ and $\epsilon$.

Proposition 7. Let $K$ be a function field with field of constants $\mathbb{F}_{q}$. Fix an effective divisor $\mathfrak{C} \in \operatorname{Div}(K)$ and a positive integer $m$. Then for all $\epsilon>0$

$$
\begin{aligned}
\sum_{\substack{[F: K]=2 \\
g_{F}=m, q_{F}=q}} \chi(F / 2 \mathfrak{C})=q^{2 m} \frac{2 J_{K} q^{3-5 g_{K}}}{\zeta_{K}(2)(q-1)} \prod_{v \in \operatorname{Supp}(\mathfrak{C})}\left(1+q^{-\operatorname{deg}(v)}\right)^{-1} \\
+ \begin{cases}O\left(q^{(1 / 2+\epsilon) m} q^{\epsilon \operatorname{deg}(\mathfrak{C})}\right) & \text { if } q \text { is odd, } \\
O\left(q^{\epsilon m} q^{\epsilon \operatorname{deg}(\mathfrak{C})}+q^{m}\right) & \text { if } q \text { is even, }\end{cases}
\end{aligned}
$$

where the implicit constants depend only on $\epsilon$ and $K$. In particular, the number $N$ of quadratic extensions $F \supset K$ with $q_{F}=q$ and $g_{F}=m$ satisfies

$$
N=q^{2 m} \frac{2 J_{K} q^{3-5 g_{K}}}{\zeta_{K}(2)(q-1)}+ \begin{cases}O\left(q^{(1 / 2+\epsilon) m}\right) & \text { if } q \text { is odd } \\ O\left(q^{m}\right) & \text { if } q \text { is even }\end{cases}
$$

The proof of Proposition 5 requires two more intermediate results.

Lemma 15. Let $K$ be a function field with $q_{K}=q$ odd and suppose $v_{0} \in M(K)$. Every quadratic extension $F$ of $K$ has a generator in $L\left(n v_{0}\right)$ for some positive integer $n$. Suppose further that $\operatorname{deg}\left(v_{0}\right)$ is odd. Let $F$ be a quadratic extension of $K$ and write

$$
2 n \operatorname{deg}\left(v_{0}\right)=\operatorname{deg}\left(\operatorname{Disc}_{K}(F)\right)+2 d
$$

for some integer $d$ satisfying $0 \leq d<\operatorname{deg}\left(v_{0}\right)$. Let $\mathfrak{A}_{1}, \ldots, \mathfrak{A}_{J_{K}}$ be representatives of the divisor classes of degree $d$. Then there is a $j$ that is uniquely determined by $v_{0}$ and $F$ such that $F$ has a generator $\omega \in L\left(2 n v_{0}-2 \mathfrak{A}_{j}\right)$; there are precisely $(q-1) / 2$ such generators $\omega$ and they all satisfy

$$
\operatorname{div}(\omega)=-2 n v_{0}+2 \mathfrak{A}_{j}+\operatorname{Disc}_{K}(F)
$$

Proof. Suppose $F$ is a quadratic extension and let $\omega_{0}$ be a generator of $F$. By the Strong Approximation Theorem there is an $\alpha \in K$ with $\operatorname{ord}_{v}(\alpha)=-\operatorname{ord}_{v}\left(\omega_{0}\right)$ for all places $v \neq v_{0}$ with 
$\operatorname{ord}_{v}\left(\omega_{0}\right)<0$ and $\operatorname{ord}_{v}(\alpha) \geq 0$ for all other places $v \neq v_{0}$. Then $\omega_{1}=\alpha^{2} \omega_{0}$ generates $F$ and $\operatorname{ord}_{v}\left(\omega_{1}\right) \geq 0$ for all places $v \neq v_{0}$ by construction; in other words, $\omega_{1} \in L\left(n v_{0}\right)$ for some positive integer $n$.

Now suppose $\operatorname{deg}\left(v_{0}\right)$ is odd. We have

$$
\operatorname{div}\left(\omega_{1}\right)=\operatorname{ord}_{v_{0}}\left(\omega_{1}\right)+\operatorname{Disc}_{K}(F)+2 \mathfrak{A}
$$

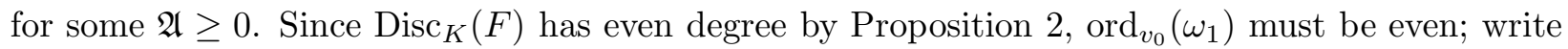
$-2 n_{1}=\operatorname{ord}_{v_{0}}\left(\omega_{1}\right)$. Since $\operatorname{deg}\left(v_{0}\right)$ is odd, by the Fundamental Theorem of Arithmetic we have $2 n \operatorname{deg}\left(v_{0}\right)=\operatorname{deg}\left(\operatorname{Disc}_{K}(F)\right)+2 d$ for uniquely determined integers $n>0$ and $0 \leq d<\operatorname{deg}\left(v_{0}\right)$. Then $2\left(n_{1}-n\right) \operatorname{deg}\left(v_{0}\right)=2 \operatorname{deg}(\mathfrak{A})-2 d$, so that $\left(n-n_{1}\right) v_{0}+\mathfrak{A}$ is a divisor of degree $d$. There is an $\alpha \in K$ and a $j$ with $\operatorname{div}(\alpha)+\left(n-n_{1}\right) v_{0}+\mathfrak{A}=\mathfrak{A}_{j}$. Now $F$ is generated by $\omega=\omega_{1} \alpha^{2}$ and

$$
\begin{aligned}
\operatorname{div}(\omega) & =\operatorname{div}\left(\omega_{1}\right)+2 \operatorname{div}(\alpha) \\
& =-2 n_{1} v_{0}+\operatorname{Disc}_{K}(F)+2 \mathfrak{A}+2 \operatorname{div}(\alpha) \\
& =-2 n v_{0}+\operatorname{Disc}_{K}(F)+2 \mathfrak{A}_{j} .
\end{aligned}
$$

If $\omega^{\prime} \in L\left(n v_{0}-2 \mathfrak{A}_{j^{\prime}}\right)$ is another generator of $F$ for some $j^{\prime}$, then $\omega^{\prime}=\omega \beta^{2}$ for some $\beta \in K$ with $2 \operatorname{div}(\beta)=\operatorname{div}\left(\beta^{2}\right)=2 \mathfrak{A}_{j^{\prime}}-2 \mathfrak{A}_{j}$, so that $\mathfrak{A}_{j^{\prime}}-\mathfrak{A}_{j}$ is a principal divisor and whence $j^{\prime}=j$.

Finally, $\omega, \omega^{\prime} \in L\left(2 n v_{0}-2 \mathfrak{A}_{j}\right)$ generate the same quadratic extension $F$ with $\operatorname{deg}\left(\operatorname{Disc}_{K}(F)\right)=$ $2 n \operatorname{deg}\left(v_{0}\right)-2 d$ if and only if $\operatorname{div}\left(\omega^{\prime}\right)=\operatorname{div}(\omega)$ by what we have already shown. This is the case if and only if $\omega^{\prime} / \omega=a \in \mathbb{F}_{q}^{\times}$, and $a$ must be a square since $\omega$ and $\omega^{\prime}$ generate the same extension. Since there are $(q-1) / 2$ squares in $\mathbb{F}_{q}^{\times}$, the proof is complete.

Lemma 16. Let $K$ be a function field with $q_{K}=q$ odd and let $\mathfrak{C} \in \operatorname{Div}(K)$ be an effective divisor. Suppose $v_{0} \in M(K)$ with $v_{0} \notin \operatorname{Supp}(\mathfrak{C})$. Then there are $\mathfrak{A}_{i, j} \in \operatorname{Div}(K)$ for $0 \leq i<\operatorname{deg}\left(v_{0}\right)$ and $1 \leq j \leq J_{K}$ with the following two properties: i) $\mathfrak{A}_{i, 1}, \ldots, \mathfrak{A}_{i, J_{K}}$ are representatives of the divisor classes of degree $i$ for all $i=1, \ldots, \operatorname{deg}\left(v_{0}\right)-1$; and ii) $\operatorname{ord}_{v}\left(\mathfrak{A}_{i, j}\right) \geq 0$ for all $v \neq v_{0}$, with equality when $v \in \operatorname{Supp}(\mathfrak{C})$, for all $i=1, \ldots, \operatorname{deg}\left(v_{0}\right)-1$ and $j=1, \ldots, J_{K}$. 
Suppose further that $\operatorname{deg}\left(v_{0}\right)$ is odd. Then

$$
\frac{q-1}{2} \sum_{\substack{[F: K]=2 \\ g_{F}=m, q_{F}=q}} \chi(F / \mathfrak{C})
$$

for all integers $m \geq 0$.

Proof. For notational convenience set $m^{\prime}=m-2 g_{K}+1$, so that

$$
\sum_{\substack{[F: K]=2 \\ g_{F}=m, q_{F}=q}} \chi(F / \mathfrak{C})=\sum_{\substack{\mathfrak{D} \in \operatorname{Div}(K) \\ \operatorname{deg}(\mathfrak{D})=2 m^{\prime}}} \sum_{\substack{[F: K]=2 \\ \operatorname{qisc}_{F}=q \\ \operatorname{Dis}_{K}(F)=\mathfrak{D}}} \chi(F / \mathfrak{C})
$$

by $\left(2^{\prime}\right)$.

Fix an $i$ and let $\mathfrak{A}_{1}, \ldots, \mathfrak{A}_{J_{K}}$ be representatives of the divisor classes of degree $i$. By the Strong Approximation Theorem, for each $j=1, \ldots, J_{K}$ there is an $\alpha_{j} \in K$ such that $\operatorname{ord}_{v}\left(\alpha_{j}\right)=$ $-\operatorname{ord}_{v}\left(\mathfrak{A}_{j}\right)$ for all places $v \neq v_{0}$ with $\operatorname{ord}_{v}\left(\mathfrak{A}_{j}\right)<0$ or $v \in \operatorname{Supp}(\mathfrak{C})$ and $\operatorname{ord}_{v}\left(\alpha_{j}\right) \geq 0$ for all other places $v \neq v_{0}$. Then for all $j=1, \ldots, J_{K}$ we have $\operatorname{ord}_{v}\left(\mathfrak{A}_{j}+\operatorname{div}\left(\alpha_{j}\right)\right) \geq 0$ for all places $v \neq v_{0}$, with equality when $v \in \operatorname{Supp}(\mathfrak{C})$. Thus for all $j=1, \ldots, J_{K}$ we have $\operatorname{ord}_{v}\left(\mathfrak{A}_{j}\right) \geq 0$ for all places $v \neq v_{0}$, with equality if $v \in \operatorname{Supp}(\mathfrak{C})$. In this manner, we see that there are $\mathfrak{A}_{i, j}$ satisfying the two properties in the lemma.

Fix an $i$ and $j$ and suppose $k \operatorname{deg}\left(v_{0}\right)-i \geq 0$. For $\alpha \in L\left(2 k v_{0}-2 \mathfrak{A}_{i, j}\right) \backslash L\left(2(k-1) v_{0}-2 \mathfrak{A}_{i, j}\right)$ we write

$$
\operatorname{div}(\alpha)=-2 k v_{0}+2 \mathfrak{A}_{i, j}+\mathfrak{A}^{\prime}+2 \mathfrak{A}^{\prime \prime}
$$

where $\mathfrak{A}^{\prime}$ and $\mathfrak{A}^{\prime \prime}$ are effective divisors, $\mathfrak{A}^{\prime}$ is square-free and $v_{0} \notin \operatorname{Supp}\left(\mathfrak{A}^{\prime \prime}\right)$. Note that $\alpha$ generates a quadratic extension $F$ with $\operatorname{Disc}_{K}(F)=\mathfrak{A}^{\prime}$ (unless $\mathfrak{A}^{\prime}=0$ ). We get an idele $\left(\alpha_{v}\right)$ given by $\alpha_{v}=\alpha / \pi_{v}^{2 \operatorname{ord}_{v}\left(\mathfrak{A}^{\prime \prime}\right)}$ for all $v \in M(K)$. Then $\operatorname{div}\left(\left(\alpha_{v}\right)\right)=-2 k v_{0}+2 \mathfrak{A}_{i, j}+\mathfrak{A}^{\prime}$.

Now suppose $\mathfrak{B}$ is an effective divisor with $0 \leq \operatorname{deg}(\mathfrak{B}) \leq m^{\prime}$ and $\left(\mathfrak{B}, \mathfrak{C}+v_{0}\right)=0$. Then there are uniquely determined $i$ and $k$ with $k \operatorname{deg}\left(v_{0}\right)-i=m^{\prime}-\operatorname{deg}(\mathfrak{B})$. For any $j=1, \ldots, J_{K}$ and $\alpha \in L\left(2 k v_{0}-2 \mathfrak{A}_{i, j}\right) \backslash L\left(2(k-1) v_{0}-\mathfrak{A}_{i, j}\right)$ we have

$$
2 \mathfrak{B}+\operatorname{div}(\alpha)=-2 k v_{0}+2 \mathfrak{A}_{i, j}+\mathfrak{A}^{\prime}+2\left(\mathfrak{A}^{\prime \prime}+\mathfrak{B}\right)=\operatorname{div}\left(\left(\alpha_{v}\right)\right)+2 \mathfrak{E},
$$


where $\mathfrak{E}$ is an effective divisor with $v_{0} \notin \operatorname{Supp}(\mathfrak{E})$ and

$$
\begin{aligned}
2 \operatorname{deg}(\mathfrak{E}) & =2 \operatorname{deg}(\mathfrak{B})+\operatorname{deg}(\operatorname{div}(\alpha))-\operatorname{deg}\left(\operatorname{div}\left(\left(\alpha_{v}\right)\right)\right) \\
& =2 \operatorname{deg}(\mathfrak{B})+2 k \operatorname{deg}\left(v_{0}\right)-2 i-\operatorname{deg}\left(\mathfrak{A}^{\prime}\right) \\
& =2 m^{\prime}-\operatorname{deg}\left(\mathfrak{A}^{\prime}\right)
\end{aligned}
$$

For any effective divisor $\mathfrak{D} \in \operatorname{Div}(K)$, set $\iota(\mathfrak{D})$ to be the idele $\left(\pi_{v}^{\operatorname{ord}_{v}(\mathfrak{D})}\right)$. By construction/definition, we then have

$$
\chi_{\mathfrak{C}}(\alpha)=\chi_{\mathfrak{C}}(\alpha \cdot \iota(2 \mathfrak{B}))=\chi_{\mathfrak{C}}\left(\left(\alpha_{v}\right) \cdot \iota(2 \mathfrak{E})\right) .
$$

With the above in mind, using (1) yields

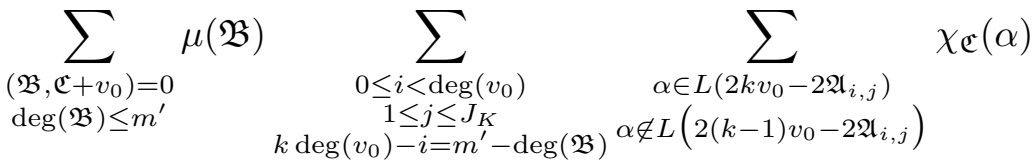

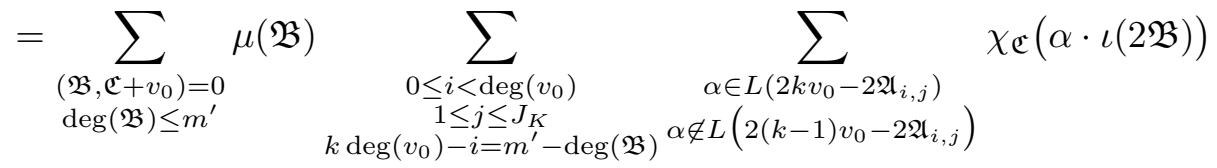

$$
\begin{aligned}
& =\sum_{\substack{\left(\mathfrak{B}, \mathfrak{C}^{\prime}+v_{0}\right)=0 \\
\operatorname{deg}(\mathfrak{B}) \leq m^{\prime}}} \sum_{\substack{0 \leq i<\operatorname{deg}\left(v_{0}\right) \\
1 \leq j \leq J_{K} \\
k \operatorname{deg}\left(v_{0}\right)-i=m^{\prime}-\operatorname{deg}(\mathfrak{B})}} \sum_{\substack{\alpha \in L\left(2 k v_{0}-2 \mathfrak{A}_{i, j}\right) \\
\alpha \notin L\left(2(k-1) v_{0}-2 \mathfrak{A}_{i, j}\right)}} \\
& \times \sum_{\begin{array}{c}
\mathfrak{B} \leq \mathfrak{E},\left(\mathfrak{E}, v_{0}\right)=0 \\
\operatorname{deg}(\mathfrak{E})=2 m^{\prime}-\operatorname{deg}\left(\mathfrak{A}^{\prime}\right)
\end{array}} \chi_{\mathfrak{C}}\left(\left(\alpha_{v}\right) \cdot \iota(2 \mathfrak{E})\right) \\
& =\sum_{\substack{\left(\mathfrak{B}, \mathfrak{C}+v_{0}\right)=0 \\
\operatorname{deg}(\mathfrak{B}) \leq m^{\prime}}} \mu(\mathfrak{B}) \sum_{\substack{0 \leq i<\operatorname{deg}\left(v_{0}\right) \\
1 \leq j \leq J_{K} \\
k \operatorname{deg}\left(v_{0}\right)-i=m^{\prime}-\operatorname{deg}(\mathfrak{B})}} \sum_{\begin{array}{c}
\alpha \in L\left(2 k v_{0}-2 \mathfrak{A}_{i, j}\right) \\
\alpha \notin L\left(2(k-1) v_{0}-2 \mathfrak{A}_{i, j}\right)
\end{array}} \\
& \times \sum_{\substack{\mathfrak{B} \leq \mathfrak{E} \\
\operatorname{deg}(\mathfrak{E})=2 m^{\prime}-\operatorname{deg}\left(\mathfrak{A}^{\prime}\right) \\
\left(\mathfrak{E}, \mathfrak{C}+v_{0}\right)=0}} \chi_{\mathfrak{C}}\left(\left(\alpha_{v}\right)\right) \\
& =\sum_{\substack{0 \leq i<\operatorname{deg}\left(v_{0}\right) \\
1 \leq j \leq J_{K} \\
k \operatorname{deg}\left(v_{0}\right)-i \leq m^{\prime}}} \sum_{\substack{\alpha \in L\left(2 k v_{0}-2 \mathfrak{A}_{i, j}\right) \\
\alpha \notin L\left(2(k-1) v_{0}-2 \mathfrak{A}_{i, j}\right)}} \chi_{\mathfrak{C}\left(\left(\alpha_{v}\right)\right)} \sum_{\substack{2 \operatorname{deg}(\mathfrak{E})=2 m^{\prime}-\operatorname{deg}\left(\mathfrak{A}^{\prime}\right) \\
\left(\mathfrak{E}, \mathfrak{C}+v_{0}\right)=0}} \mu(\mathfrak{B}) \\
& =\sum_{\substack{0 \leq i<\operatorname{deg}\left(v_{0}\right) \\
1 \leq j \leq J_{K} \\
k \operatorname{deg}\left(v_{0}\right)-i \leq m^{\prime}}} \sum_{\substack{\alpha \in L\left(2 k v_{0}-2 \mathfrak{A}_{i, j}\right) \\
\alpha \notin L\left(2(k-1) v_{0}-2 \mathfrak{A}_{i, j}\right) \\
\operatorname{deg}\left(\mathfrak{A}^{\prime}\right)=2 m^{\prime}}} \chi_{\mathfrak{C}}\left(\left(\alpha_{v}\right)\right) .
\end{aligned}
$$

Now if $\alpha \in L\left(2 k v_{0}-2 \mathfrak{A}_{i, j}\right) \backslash L\left(2(k-1) v_{0}-2 \mathfrak{A}_{i, j}\right)$, then $\operatorname{deg}\left(\mathfrak{A}^{\prime}\right)+2 \operatorname{deg}\left(\mathfrak{A}^{\prime \prime}\right)=2 k \operatorname{deg}\left(v_{0}\right)-2 i$. Therefore, if $\operatorname{deg}\left(\mathfrak{A}^{\prime}\right)=2 m^{\prime}$ and $k \operatorname{deg}\left(v_{0}\right)-i \leq m^{\prime}$, we must have $k \operatorname{deg}\left(v_{0}\right)-i=m^{\prime}$ and $\mathfrak{A}^{\prime \prime}=0$, 
so that the idele $\left(\alpha_{v}\right)$ is simply $\alpha$. Thus by Proposition 4 , if $F$ denotes the quadratic extension generated by $\alpha$, we have $\chi(F / \mathfrak{C})=\chi_{\mathfrak{C}}(\alpha)$ and $\operatorname{deg}\left(\operatorname{Disc}_{K}(F)\right)=2 m^{\prime}$. Applying Lemma 15 finishes the proof.

Proof of Proposition 5. Write $m^{\prime}=m-2 g_{K}+1$ as above.

Suppose first that $m \leq \operatorname{deg}(\mathfrak{C}) / 4$. The estimate in Proposition 5 in this case is the trivial one given Proposition 7, but we can also prove it independently by Lemma 16. Choose any place $v_{0} \in M(K)$ of odd degree. Then by Lemma 16 and (0)

$$
\begin{aligned}
\sum_{\substack{[F: K]=2 \\
g_{F}=m, q_{F}=q}} \chi(F / \mathfrak{C}) \mid & \sum_{\substack{\mathfrak{B} \geq 0 \\
\operatorname{deg}(\mathfrak{B}) \leq m^{\prime}}} \sum_{\substack{\mathfrak{B} \geq 0 \\
0 \leq i \leq \operatorname{deg}\left(v_{0}\right) \\
1 \leq j \leq J_{K} \\
k \operatorname{deg}\left(v_{0}\right)-i=m^{\prime}-\operatorname{deg}(\mathfrak{B})}} \sum_{\substack{\mathfrak{B} \geq 0 \\
\operatorname{deg}(\mathfrak{B}) \leq m^{\prime}}} \sum_{\substack{0 \leq i \leq \operatorname{deg}\left(v_{0}\right) \\
1 \leq j \leq J_{K} \\
k \operatorname{deg}\left(v_{0}\right)-i=m^{\prime}-\operatorname{deg}(\mathfrak{B})}} q^{2 k \operatorname{deg}\left(v_{0}\right)-2 i} \\
& \ll q^{2 m^{\prime}} \sum_{\substack{\mathfrak{B} \geq 0 \\
\operatorname{deg}(\mathfrak{B}) \leq m^{\prime}}} q^{-2 \operatorname{deg}(\mathfrak{B})} \\
& \ll q^{2 m^{\prime}} \\
& \ll q^{2 m} .
\end{aligned}
$$

For the case where $m>\operatorname{deg}(\mathfrak{C}) / 4$ we will again use Lemma 16 , but we must be more careful in our choice of the place $v_{0}$ and the divisors $\mathfrak{A}_{i, j}$.

A weak corollary to the "prime number theorem" for function fields (see [8, Theorem 5.12], for example) together with Lemma 0 implies that there is a place $v_{0} \in M(K)$ of odd degree with $v_{0} \notin \operatorname{Supp}(\mathfrak{C})$ satisfying $q^{\operatorname{deg}\left(v_{0}\right)} \ll q^{\epsilon \operatorname{deg}(\mathfrak{C})}$, where the implicit constant depends only on $K$ and $\epsilon$.

Next, for all $k$ with $k \operatorname{deg}\left(v_{0}\right) \geq 2 g_{K}-1+\operatorname{deg}(\mathfrak{C})$ we have

$$
\begin{aligned}
\#\left\{\alpha \in L\left(k v_{0}\right): \operatorname{ord}_{v}(\alpha)=0 \text { all } v \in \operatorname{Supp}(\mathfrak{C})\right\} & =\sum_{0 \leq \mathfrak{B} \leq \mathfrak{C}} \mu(\mathfrak{B}) \# L\left(k v_{0}-\mathfrak{B}\right) \\
& =q^{k \operatorname{deg}\left(v_{0}\right)+1-g_{K}} \sum_{0 \leq \mathfrak{B} \leq \mathfrak{C}} \mu(\mathfrak{B}) q^{-\operatorname{deg}(\mathfrak{B})} \\
& =q^{k \operatorname{deg}\left(v_{0}\right)+1-g_{K}} \prod_{v \in \operatorname{Supp}(\mathfrak{C})}\left(1-q^{-\operatorname{deg}(v)}\right) .
\end{aligned}
$$

In particular, we see that for all integers $k$ with $k \operatorname{deg}\left(v_{0}\right) \geq 2 g_{K}+\operatorname{deg}(\mathfrak{C})$, there is an $\alpha \in K$ with $\operatorname{ord}_{v_{0}}(\alpha)=-k$ and $\operatorname{ord}_{v}(\alpha) \geq 0$ for all places $v \neq v_{0}$, with equality whenever $v \in \operatorname{Supp}(\mathfrak{C})$. Hence 
there is a positive integer $n$ such that we can choose divisors $\mathfrak{A}_{i, j}$ satisfying the two properties of Lemma 16 and also $\operatorname{ord}_{v_{0}}\left(\mathfrak{A}_{i, j}\right)=-n$ for all $0 \leq i<\operatorname{deg}\left(v_{0}\right)$ and $1 \leq j \leq J_{K}$. Set $\mathfrak{D}_{i, j}=\mathfrak{A}_{i, j}+n v_{0}$. Then $\mathfrak{D}_{i, j}$ is always an effective divisor with $\left(\mathfrak{C}+v_{0}, \mathfrak{D}_{i, j}\right)=0$ and $L\left(2 k v_{0}-2 \mathfrak{A}_{i, j}\right)=L(2(k+$ n) $\left.v_{0}-2 \mathfrak{D}_{i, j}\right)$.

Clearly $\chi_{\mathfrak{C}}$ induces a multiplicative character $\Phi$ on $\mathfrak{O}_{\mathbb{A}}(\mathfrak{C}) / \pi_{\mathbb{A}}(\mathfrak{C})$ and $\Phi$ is non-principal since $\mathfrak{C} \notin 2 \operatorname{Div}(K)$. Letting $\theta_{\mathfrak{C}}: \mathfrak{O}_{\mathbb{A}}(\mathfrak{C}) \rightarrow \mathfrak{O}_{\mathbb{A}}(\mathfrak{C}) / \pi_{\mathbb{A}}(\mathfrak{C})$ denote the canonical map as in the proof of Theorem 4, we have $\operatorname{ker}\left(\theta_{\mathfrak{C}}\right) \cap L\left(2(k+n) v_{0}-2 \mathfrak{D}_{i, j}\right)=L\left(2(k+n) v_{0}-2 \mathfrak{D}_{i, j}-\mathfrak{C}\right)$ as explained in the proof of Lemma 10. Now either $2(k+n) \operatorname{deg}\left(v_{0}\right)-2 i \geq \operatorname{deg}(\mathfrak{C}) 2 g_{K}-1$, in which case

$$
\sum_{\alpha \in L\left(2(k+n) v_{0}-2 \mathfrak{D}_{i, j}\right)} \chi_{\mathfrak{C}}(\alpha)=\# L\left(2(k+n) v_{0}-2 \mathfrak{D}_{i, j}-\mathfrak{C}\right) \sum_{r \in \mathfrak{O}_{\mathbb{A}}(\mathfrak{C}) / \pi_{\mathbb{A}}(\mathfrak{C})} \Phi(r)=0
$$

by Theorem 4 , or $2(k+n) \operatorname{deg}\left(v_{0}\right)-2 i<\operatorname{deg}(\mathfrak{C})+2 g_{K}-1$, in which case

$$
\begin{aligned}
\sum_{\alpha \in L\left(2(k+n) v_{0}-2 \mathfrak{D}_{i, j}\right)} \chi_{\mathfrak{C}}(\alpha) \mid & =\# L\left(2(k+n) v_{0}-2 \mathfrak{D}_{i, j}-\mathfrak{C}\right)\left|\sum_{r \in \theta_{\mathfrak{C}}\left(L\left(2(k+n)-2 \mathfrak{D}_{i, j}\right)\right)} \Phi(r)\right| \\
& \ll \min \left\{q^{2(k+n) \operatorname{deg}\left(v_{0}\right)-\operatorname{deg}\left(\mathfrak{D}_{i, j}\right)}, q^{\operatorname{deg}(\mathfrak{C}) / 2+\operatorname{deg}\left(v_{0}\right)}\right\} \\
& =\min \left\{q^{2 k \operatorname{deg}\left(v_{0}\right)-2 i}, q^{\operatorname{deg}(\mathfrak{C}) / 2+\operatorname{deg}\left(v_{0}\right)}\right\} \\
& \ll \min \left\{q^{2 k \operatorname{deg}\left(v_{0}\right)-2 i}, q^{\operatorname{deg}(\mathfrak{C})(\epsilon+1 / 2)}\right\}
\end{aligned}
$$

by Theorem 4 again. We thus have

$$
\begin{aligned}
\left|\sum_{\substack{\alpha \in L\left(2 k v_{0}-2 \mathfrak{A}_{i, j}\right) \\
\alpha \notin L\left(2(k-1) v_{0}-2 \mathfrak{A}_{i, j}\right)}} \chi_{\mathfrak{C}}(\alpha)\right| \leq\left|\sum_{\alpha \in L\left(2 k v_{0}-2 \mathfrak{A}_{i, j}\right)} \chi_{\mathfrak{C}}(\alpha)\right|+\left|\sum_{\alpha \in L\left(2(k-1) v_{0}-2 \mathfrak{A}_{i, j}\right)} \chi_{\mathfrak{C}}(\alpha)\right| \\
\ll \min \left\{q^{2 k \operatorname{deg}\left(v_{0}\right)-2 i}, q^{\operatorname{deg}(\mathfrak{C})(\epsilon+1 / 2)}\right\} .
\end{aligned}
$$


Using this estimate together with Lemma 16, we get

$$
\begin{aligned}
& \left|\sum_{\substack{[F: K]=2 \\
g_{F}=m, q_{F}=q}} \chi(F / \mathfrak{C})\right| \leq\left|\sum_{\substack{\left(\mathfrak{B}, \mathfrak{C}+v_{0}\right)=0 \\
m^{\prime} \geq \operatorname{deg}(\mathfrak{B}) \geq m^{\prime}-\operatorname{deg}(\mathfrak{C}) / 4}} \mu(\mathfrak{B}) \sum_{\substack{0 \leq i<\operatorname{deg}\left(v_{0}\right) \\
1 \leq j \leq J_{K} \\
k \operatorname{deg}\left(v_{0}\right)-i=m^{\prime}-\operatorname{deg}(\mathfrak{B})}} \sum_{\substack{\alpha \notin L\left(2 k v_{0}-2 \mathfrak{A}_{i, j}\right) \\
\alpha \notin L\left(2(k-1) v_{0}-2 \mathfrak{A}_{i, j}\right)}} \chi_{\mathfrak{C}}(\alpha)\right| \\
& +\left|\sum_{\substack{\left(\mathfrak{B}, \mathfrak{C}+v_{0}\right)=0 \\
0 \leq \operatorname{deg}(\mathfrak{B})<m^{\prime}-\operatorname{deg}(\mathfrak{C}) / 4}} \mu(\mathfrak{B}) \sum_{\substack{0 \leq i<\operatorname{deg}\left(v_{0}\right) \\
1 \leq j \leq J_{K} \\
k \operatorname{deg}\left(v_{0}\right)-i=m^{\prime}-\operatorname{deg}(\mathfrak{B})}} \sum_{\substack{\alpha \in L\left(2 k v_{0}-2 \mathfrak{A}_{i, j}\right) \\
\alpha \notin L\left(2(k-1) v_{0}-2 \mathfrak{A}_{i, j}\right)}} \chi_{\mathfrak{C}}(\alpha)\right| \\
& \leq \sum_{\substack{\left(\mathfrak{B}, \mathfrak{C}+v_{0}\right)=0 \\
m^{\prime} \geq \operatorname{deg}(\mathfrak{B}) \geq m^{\prime}-\operatorname{deg}(\mathfrak{C}) / 4}} \sum_{\substack{0 \leq i<\operatorname{deg}\left(v_{0}\right) \\
1 \leq j \leq J_{K} \\
k \operatorname{deg}\left(v_{0}\right)-i=m^{\prime}-\operatorname{deg}(\mathfrak{B})}} \sum_{\substack{\alpha \in L\left(2 k v_{0}-2 \mathfrak{A}_{i, j}\right) \\
\alpha \notin L\left(2(k-1) v_{0}-2 \mathfrak{A}_{i, j}\right)}} \chi_{\mathfrak{C}}(\alpha) \mid \\
& +\sum_{\substack{\left(\mathfrak{B}, \mathfrak{C}+v_{0}\right)=0 \\
0 \leq \operatorname{deg}(\mathfrak{B})<m^{\prime}-\operatorname{deg}(\mathfrak{C}) / 4}}\left|\sum_{\substack{0 \leq i<\operatorname{deg}\left(v_{0}\right) \\
1 \leq j \leq J_{K} \\
k \operatorname{deg}\left(v_{0}\right)-i=m^{\prime}-\operatorname{deg}(\mathfrak{B})}} \chi_{\substack{\alpha \in L\left(2 k v_{0}-2 \mathfrak{A}_{i, j}\right) \\
\alpha \notin L\left(2(k-1) v_{0}-2 \mathfrak{A}_{i, j}\right)}} \chi_{\mathfrak{C}}(\alpha)\right| \\
& \ll \sum_{\left(\mathfrak{B}, \mathfrak{C}+v_{0}\right)=0} q^{2 m^{\prime}-2 \operatorname{deg}(\mathfrak{B})} \\
& m^{\prime} \geq \operatorname{deg}(\mathfrak{B}) \geq m^{\prime}-\operatorname{deg}(\mathfrak{C}) / 4 \\
& +\sum_{\substack{\left(\mathfrak{B}, \mathfrak{C}+v_{0}\right)=0 \\
0 \leq \operatorname{deg}(\mathfrak{B})<m^{\prime}-\operatorname{deg}(\mathfrak{C}) / 4}} q^{\operatorname{deg}(\mathfrak{C})(\epsilon+1 / 2)} \\
& \ll q^{2 m^{\prime}} q^{-m^{\prime}+\operatorname{deg}(\mathfrak{C}) / 4}+q^{m^{\prime}-\operatorname{deg}(\mathfrak{C}) / 4} q^{\operatorname{deg}(\mathfrak{C})(\epsilon+1 / 2)} \\
& \ll q^{m+(\epsilon+1 / 4) \operatorname{deg}(\mathfrak{C})} .
\end{aligned}
$$

We now turn to the case of characteristic two and Proposition 6. We first note that by Lemma 9 it suffices to prove Proposition 6 under the assumption that $\operatorname{deg}(\mathfrak{C})$ is even. Before we can finish the proof, we need several intermediate results.

Lemma 17. Suppose $K$ is a function field with $q_{K}=q$ even and $\mathfrak{C}$ is an effective divisor with $\mathfrak{C} \notin 2 \operatorname{Div}(K)$. Then the function $\chi_{\mathfrak{C}}$ is a non-trivial character on $\mathfrak{O}_{\mathbb{A}}(\mathfrak{C})$. Further, $L(0) \subseteq \operatorname{ker}\left(\chi_{\mathfrak{C}}\right)$ if and only if $\operatorname{deg}(\mathfrak{C})$ is even. For each place $v \notin \operatorname{Supp}(\mathfrak{C})$, the supremum $\sup \left\{n: L(n v) \subseteq \operatorname{ker}\left(\chi_{\mathfrak{C}}\right)\right\}$ is an achieved maximum; call it $n_{v}$ and set $n_{v}=0$ for all $v \in \operatorname{Supp}(\mathfrak{C})$. For any effective divisor $\mathfrak{A}$ 
with $(\mathfrak{A}, \mathfrak{C})=0, L(\mathfrak{A}) \subseteq \operatorname{ker}\left(\chi_{\mathfrak{C}}\right)$ only if $\operatorname{ord}_{v}(\mathfrak{A}) \leq n_{v}$ for all $v \in M(K)$. Finally, if $\operatorname{deg}(\mathfrak{C})$ is even then we get an effective divisor

$$
\mathfrak{C}_{\chi}=\sum_{v \in M(K)} n_{v} v
$$

and for all effective divisors $\mathfrak{A}$ with $(\mathfrak{A}, \mathfrak{C})=0, L(\mathfrak{A}) \subseteq \operatorname{ker}\left(\chi_{\mathfrak{C}}\right)$ only if $\mathfrak{A} \leq \mathfrak{C}_{\chi}$.

Proof. Clearly $\chi_{\mathfrak{C}}$ is a character on $\mathfrak{O}_{\mathbb{A}}(\mathfrak{C})$ from the definitions, with $\operatorname{ker}\left(\chi_{\mathfrak{C}}\right) \supseteq \pi_{\mathbb{A}}(\mathfrak{C})$. Since $\chi_{v}$ is certainly non-trivial for all places $v \in M(K)$, we see that $\chi_{\mathfrak{C}}$ is non-trivial whenever $\mathfrak{C} \notin 2 \operatorname{Div}(K)$ by the definitions.

Next, $L(0)=\mathbb{F}_{q}$. Clearly all $a \in \mathbb{F}_{q}$ of the form $a=b^{2}+b$ for some $b \in \mathbb{F}_{q}$ satisfy $\chi_{v}(a)=1$ for all places $v \in M(K)$. Thus by Lemma 11 we see that $L(0)=\mathbb{F}_{q} \subseteq \operatorname{ker}\left(\chi_{\mathfrak{C}}\right)$ if and only if $\operatorname{deg}(\mathfrak{C})$ is even.

Note that $L(-v)=\{0\}$ for all places $v \in M(K)$, so that $n_{v} \geq-1$ always. If $v \notin \operatorname{Supp}(\mathfrak{C})$, then $L(n v) \not \subset \operatorname{ker}\left(\chi_{\mathfrak{C}}\right)$ for all positive integers $n$ with $n \operatorname{deg}(v) \geq \operatorname{deg}\left(\mathfrak{C}_{1}\right)+2 g_{K}-1$ by Lemma 10, since $\chi_{\mathfrak{C}}$ is non-trivial with $\operatorname{ker}\left(\chi_{\mathfrak{C}}\right) \supseteq \pi_{\mathbb{A}}(\mathfrak{C})$. Thus $n_{v}$ is always an achieved maximum.

Now suppose $\mathfrak{A}$ is an effective divisor with $(\mathfrak{A}, \mathfrak{C})=0$ and $L(\mathfrak{A}) \subseteq \operatorname{ker}\left(\chi_{\mathfrak{C}}\right)$. Let $v \in \operatorname{Supp}(\mathfrak{A})$. Then since $\mathfrak{A}$ is an effective divisor $L\left(\operatorname{ord}_{v}(\mathfrak{A}) v\right) \subseteq L(\mathfrak{A}) \subseteq \operatorname{ker}\left(\chi_{\mathfrak{C}}\right)$, so that $\operatorname{ord}_{v}(\mathfrak{A}) \leq n_{v}$.

Finally, $n_{v} \leq 0$ for all but finitely many places $v \in M(K)$ (indeed, for all places of degree at least $\left.\operatorname{deg}\left(\mathfrak{C}_{1}\right)+2 g_{K}-1\right)$, whence $n_{v}=0$ for all but finitely many places whenever $L(0) \subseteq \operatorname{ker}\left(\chi_{\mathfrak{C}}\right)$.

Lemma 18. Suppose $K$ is a function field with $q_{K}=q$ even and $\mathfrak{D}$ is an effective divisor with $\operatorname{deg}\left(\mathfrak{D}_{2}\right) \geq 2 g_{K}-1$. Then every $F \in S(\mathfrak{D})$ has $\frac{q^{\operatorname{deg}\left(\mathfrak{D}_{2}\right)}}{2}$ generators in $L^{\prime}\left(\mathfrak{D}_{1}+2 \mathfrak{D}_{2}\right)$ and $N(\mathfrak{D})=$ $2 \phi(\mathfrak{D})$. In particular, for all effective divisors $\mathfrak{C}$ with $(\mathfrak{C}, \mathfrak{D})=0$ we have

$$
\begin{aligned}
\frac{q^{\operatorname{deg}\left(\mathfrak{D}_{2}\right)}}{2} \sum_{\substack{[F: K]=2, q_{F}=q \\
\operatorname{Disc}_{K}(F)=2 \mathfrak{D}}} \chi(F / \mathfrak{C}) & =\sum_{\omega \in L^{\prime}\left(\mathfrak{D}_{1}+2 \mathfrak{D}_{2}\right)} \chi_{\mathfrak{C}}(\alpha) \\
& =\sum_{0 \leq \mathfrak{A} \leq \mathfrak{D}_{1}+2 \mathfrak{D}_{2}} \mu(\mathfrak{A}) \sum_{\alpha \in L\left(\mathfrak{D}_{1}+2 \mathfrak{D}_{2}-\mathfrak{A}\right)} \chi_{\mathfrak{C}}(\alpha) .
\end{aligned}
$$

Proof. The first part follows directly from Lemma 5 and Proposition 3. The last part follows from the first part, Proposition 4 and a simple application of Möbius inversion using (1). 
Lemma 19. Suppose $K$ is a function field with $q_{K}=q$ even and $\mathfrak{C}$ is an effective divisor with $\mathfrak{C} \notin 2 \operatorname{Div}(K)$. Suppose $\mathfrak{D}$ is an effective divisor with $(\mathfrak{C}, \mathfrak{D})=0$ and $\operatorname{deg}\left(\mathfrak{D}_{2}\right) \geq 2 g_{K}-1$. If $\operatorname{deg}(\mathfrak{C})$ is even and $2 \mathfrak{D}_{2} \not \leq \mathfrak{C}_{\chi}$, then

$$
\sum_{\substack{[F: K]=2, \operatorname{Disc}_{K}(F)=2 \mathfrak{D}}} \chi(F / \mathfrak{C})=0
$$

Proof. Since $\operatorname{deg}\left(\mathfrak{D}_{2}\right) \geq 2 g_{K}-1$ we may use Lemma 18. Assume $\operatorname{deg}(\mathfrak{C})$ is even and $2 \mathfrak{D}_{2} \not \leq \mathfrak{C}_{\chi}$. We note that if $0 \leq \mathfrak{A} \leq \mathfrak{D}_{1}+2 \mathfrak{D}_{2}$, then $\mu(\mathfrak{A})=0$ unless $\mathfrak{A} \leq \mathfrak{D}_{1}$. Moreover, if $\mathfrak{A} \leq \mathfrak{D}_{1}$, then $\mathfrak{D}_{1}+2 \mathfrak{D}_{2}-\mathfrak{A} \geq 2 \mathfrak{D}_{2}$ and by Lemma 17 we have $L\left(2 \mathfrak{D}_{2}\right) \subseteq L\left(\mathfrak{D}_{1}+2 \mathfrak{D}_{2}-\mathfrak{A}\right) \nsubseteq \operatorname{ker}\left(\chi_{\mathfrak{C}}\right)$ since $2 \mathfrak{D}_{2} \not \leq \mathfrak{C}_{\chi}$. We thus have

$$
\begin{aligned}
\frac{q^{\operatorname{deg}\left(\mathfrak{D}_{2}\right)}}{2} \sum_{\substack{[F: K]=2, q_{F}=q_{K} \\
\operatorname{Disc}_{K}(F)=2 \mathfrak{D}}} \chi(F / \mathfrak{C}) & =\sum_{0 \leq \mathfrak{A} \leq \mathfrak{D}_{1}+2 \mathfrak{D}_{2}} \mu(\mathfrak{A}) \sum_{\alpha \in L\left(\mathfrak{D}_{1}+2 \mathfrak{D}_{2}-\mathfrak{A}\right)} \chi \mathfrak{C}(\alpha) \\
& =\sum_{0 \leq \mathfrak{A} \leq \mathfrak{D}_{1}} \mu(\mathfrak{A}) \sum_{\alpha \in L\left(\mathfrak{D}_{1}+2 \mathfrak{D}_{2}-\mathfrak{A}\right)} \chi_{\mathfrak{C}}(\alpha) \\
& =\sum_{0 \leq \mathfrak{A} \leq \mathfrak{D}_{1}} \mu(\mathfrak{A}) \cdot 0 \\
& =0 .
\end{aligned}
$$

Lemma 20. Suppose $K$ is a function field with $q_{K}=q$ even and let $\mathfrak{C}, \mathfrak{D}$ be effective divisors with $(\mathfrak{C}, \mathfrak{D})=0$ and $\mathfrak{C} \notin 2 \operatorname{Div}(K)$. Let $v_{0} \in M(K)$ with $v_{0} \notin \operatorname{Supp}(\mathfrak{C}) \cup \operatorname{Supp}(\mathfrak{D})$ and $\operatorname{deg}\left(v_{0}\right) \geq 2 g_{K}-1$. If $\mathfrak{D}>0$, then

$$
\sum_{i=0}^{\left[n_{v_{0}} / 2\right]+1} \sum_{\substack{[F: K]=2, \operatorname{Disc}_{K}(F)=2 \mathfrak{D}+2 i v_{0}}} \chi(F / \mathfrak{C})=0
$$

If $\mathfrak{D}=0$, then

$$
\sum_{i=1}^{\left[n_{v_{0}} / 2\right]+1} \sum_{\substack{[F: K]=2, q_{F}=q \\ \operatorname{Disc}_{K}(F)=2 i v_{0}}} \chi(F / \mathfrak{C})=-1-(-1)^{\operatorname{deg}(\mathfrak{C})}
$$

Proof. Suppose $\mathfrak{D}>0$. As in the proof of Proposition 3, we may choose a positive integer $n$ such that all $F \in S(\mathfrak{D}) \cup S\left(\mathfrak{D}+v_{0}\right)$ have a generator in

$$
S=\left\{\omega \in L\left(\mathfrak{D}_{1}+2 \mathfrak{D}_{2}+2 n v_{0}: \operatorname{ord}_{v}(\omega)=1-2 \operatorname{ord}_{v}(\mathfrak{D}) \text { for all } v \in \operatorname{Supp}(\mathfrak{D})\right\}\right.
$$


We may assume without loss of generality that $n \geq\left[n_{v_{0}}\right] / 2+1$. From the proof of Proposition 3 we have

$$
\frac{2}{q^{l\left(\mathfrak{D}_{2}+n v_{0}\right)}} \sum_{\omega \in S} \chi_{\mathfrak{C}}(\omega)=\sum_{i=0}^{n} \sum_{\substack{[F: K]=2, q_{F}=q \\ \operatorname{Disc}_{K}(F)=2 \mathfrak{D}+2 i v_{0}}} \chi(F / \mathfrak{C}) .
$$

On the other hand, by Möbius inversion exactly as in the proof of Lemma 4 we have

$$
\sum_{\omega \in S} \chi_{\mathfrak{C}}(\omega)=\sum_{0 \leq \mathfrak{A} \leq \mathfrak{D}_{1}+2 \mathfrak{D}_{2}} \mu(\mathfrak{A}) \sum_{\alpha \in L\left(\mathfrak{D}_{1}+2 \mathfrak{D}_{2}+2 n v_{0}-\mathfrak{A}\right)} \chi_{\mathfrak{C}}(\alpha)
$$

Since $L\left(\mathfrak{D}_{1}+2 \mathfrak{D}_{2}+2 n v_{0}-\mathfrak{A}\right) \supseteq L\left(2 n v_{0}\right)$ for all divisors $\mathfrak{A}$ with $0 \leq \mathfrak{A} \leq \mathfrak{D}_{1}+2 \mathfrak{D}_{2}$ and $2 n>n_{v_{0}}$, we have $L\left(\mathfrak{D}-1+2 \mathfrak{D}_{2}+2 n v_{0}-\mathfrak{A}\right) \nsubseteq \operatorname{ker}\left(\chi_{\mathfrak{C}}\right)$, so that

$$
\sum_{\omega \in S} \chi_{\mathfrak{C}}(\omega)=0
$$

by (8).

Now suppose $n \geq i>\left[n_{v_{0}} / 2\right]+1$. We note that $\left(\mathfrak{D}+i v_{0}\right)_{2}=\mathfrak{D}_{2}+(i-1) v_{0}$. Since $\operatorname{deg}\left((i-1) v_{0}\right) \geq$ $\operatorname{deg}\left(v_{0}\right) \geq 2 g_{K}-1$ and $2(i-1)>n_{v_{0}}$, we get

$$
\sum_{\substack{[F: K]=2, q_{F}=q_{K} \\ \operatorname{Disc}_{K}(F)=2 \mathfrak{D}+2 i v_{0}}} \chi(F / \mathfrak{C})=0
$$

by Lemma 19. The case where $\mathfrak{D}>0$ now follows from (11)-(13).

Suppose now that $\mathfrak{D}=0$. We still have (12) and (13), but now $S=L\left(2 n v_{0}\right)$ and (as remarked in the proof of Proposition 3) contains $q^{l\left(n v_{0}\right)} / 2$ elements $\alpha$ of the form $\alpha=\beta^{2}+\beta$ and $q^{l\left(n v_{0}\right)} / 2$ generators of the field $K(a)$, where $a \in \mathbb{F}_{q}$ is as in Lemma 11 . We obviously have $\chi_{\mathfrak{c}}\left(\beta^{2}+\beta\right)=1$, and $\chi_{\mathfrak{C}}(a)=(-1)^{\operatorname{deg}(\mathfrak{C})}$ by Lemma 11 . Thus we replace $(11)$ with

$$
\frac{2}{q^{l\left(n v_{0}\right)}} \sum_{\alpha \in S} \chi_{\mathfrak{C}}(\alpha)=1+(-1)^{\operatorname{deg}(\mathfrak{C})}+\sum_{i=1}^{n} \sum_{\substack{[F: K]=2, q_{F}=q \\ \operatorname{Disc}_{K}(F)=2 \mathfrak{D}+2 i v_{0}}} \chi(F / \mathfrak{C}) .
$$

The lemma follows.

Lemma 21. Suppose $K$ is a function field with $q_{K}=q$ even and $\mathfrak{C}$ is an effective divisor with $\mathfrak{C} \notin 2 \operatorname{Div}(K)$. Let $\mathfrak{D}$ be an effective divisor with $(\mathfrak{C}, \mathfrak{D})=0$ of the form

$$
\mathfrak{D}=\mathfrak{D}^{\prime}+v_{1}+\cdots+v_{m},
$$


where all places $v \in \operatorname{Supp}\left(\mathfrak{D}^{\prime}\right)$ satisfy $\operatorname{deg}(v)<2 g_{K}-1$ and the $v_{1}, \ldots, v_{m}$ are distinct places with $\operatorname{deg}\left(v_{i}\right) \geq 2 g_{K}-1$ for all $i=1, \ldots, m$. If $\mathfrak{D}^{\prime}>0$, then

$$
\begin{aligned}
& \sum_{\substack{[F: K]=2, q_{F}=q \\
\operatorname{Disc}_{K}(F)=2 \mathfrak{D}}} \chi(F / \mathfrak{C})=(-1)^{m} \sum_{\substack{[F: K]=2, q_{F}=q \\
\operatorname{Disc}_{K}(F)=2 \mathfrak{D}^{\prime}}} \chi(F / \mathfrak{C}) \\
& +\sum_{i=1}^{m}(-1)^{m-i-1} \sum_{j=2}^{\left[n_{v_{i}} / 2\right]+1} \sum_{\begin{array}{c}
{[F: K]=2, q_{F}=q} \\
\operatorname{Disc}_{K}(F)=2 \mathfrak{D}^{\prime}+2 v_{1}+\cdots+2 v_{i-1}+2 j v_{i}
\end{array}} \chi(F / \mathfrak{C}) .
\end{aligned}
$$

In particular, if $n_{v_{m}} \leq 0$, then

$$
\sum_{\substack{[F: K]=2, q_{F}=q \\
\operatorname{Disc}_{K}(F)=2 \mathfrak{D}}} \chi(F / \mathfrak{C})=-\sum_{\begin{array}{c}
{[F: K]=2, q_{F}=q} \\
\operatorname{Disc}_{K}(F)=2\left(\mathfrak{D}-v_{m}\right)
\end{array}} \chi(F / \mathfrak{C})
$$

If $\mathfrak{D}^{\prime}=0$, then

$$
\begin{aligned}
\sum_{\substack{[F: K]=2, q_{F}=q \\
\operatorname{Disc}_{K}(F)=2 \mathfrak{D}}} \chi(F / \mathfrak{C}) & =(-1)^{m}\left(1+(-1)^{\operatorname{deg}(\mathfrak{C})}\right) \\
& +\sum_{i=1}^{m}(-1)^{m-i-1} \sum_{j=2}^{\left[n_{v_{i}} / 2\right]+1} \sum_{\substack{[F: K]=2, q_{F}=q \\
\operatorname{Disc}_{K}(F)=2 v_{1}+\cdots+2 v_{i-1}+2 j v_{i}}} \chi(F / \mathfrak{C}) .
\end{aligned}
$$

In particular, if $n_{v_{m}} \leq 0$, then

$$
\sum_{\substack{[F: K]=2, q_{F}=q \\
\operatorname{Disc}_{K}(F)=2 \mathfrak{D}}} \chi(F / \mathfrak{C})=-\sum_{\begin{array}{c}
{[F: K]=2, q_{F}=q} \\
\operatorname{Disc}_{K}(F)=2\left(\mathfrak{D}-v_{m}\right)
\end{array}} \chi(F / \mathfrak{C})
$$

if $m>1$, and if $m=1$

$$
\sum_{\substack{[F: K]=2, q_{F}=q \\ \operatorname{Disc}_{K}(F)=2 v_{1}}} \chi(F / \mathfrak{C})=-1-(-1)^{\operatorname{deg}(\mathfrak{C})}
$$

Proof. First assume $\mathfrak{D}^{\prime}>0$. We prove the lemma by induction on $m$. The case $m=1$ is just Lemma 20 with the sums reorganized. Now assume $m>1$ and the lemma is true for $m-1$. By Lemma 20 we have

$$
\begin{aligned}
& \sum_{\substack{[F: K]=2, q_{F=q} \\
\operatorname{Disc}_{K}(F)=2 \mathfrak{D}}} \chi(F / \mathfrak{C})=-\sum_{\begin{array}{c}
{[F: K]=2, q_{F}=q} \\
\operatorname{Disc}_{K}(F)=2 \mathfrak{D}^{\prime}+2 v_{1}+\cdots+2 v_{m-1}
\end{array}} \chi(F / \mathfrak{C}) \\
& -\sum_{j=2}^{\left[n_{\left.v_{m} / 2\right]+1}\right.} \sum_{\substack{[F: K]=2, q_{F}=q \\
\left[\mathfrak{D}^{\prime}+2 v_{1}+\cdots+2 v_{m-1}+2 j v_{m}\right.}} \chi(F / \mathfrak{C}) .
\end{aligned}
$$


Now by the case $m-1$,

$$
\begin{array}{ccc}
\sum_{\substack{[F: K]=2, q_{F}=q \\
\operatorname{Disc}_{K}(F)=2 \mathfrak{D}^{\prime}+2 v_{1}+\cdots+2 v_{m-1}}} \chi(F / \mathfrak{C})=(-1)^{m-1} \sum_{\substack{[F: K]=2, q_{F}=q \\
\operatorname{Disc}_{K}(F)=2 \mathfrak{D}^{\prime} \\
\left[n_{\left.v_{i} / 2\right]+1}\right.}} \chi(F / \mathfrak{C}) \\
-\sum_{i=1}^{m-1}(-1)^{m-i-1} \sum_{j=0} \sum_{\substack{[F: K]=2, q_{F}=q \\
\operatorname{Disc}_{K}(F)=2 \mathfrak{D}^{\prime}+2 v_{1}+\cdots+2 v_{i-1}+2 j v_{i}}} \chi(F / \mathfrak{C}) .
\end{array}
$$

This proves the lemma when $\mathfrak{D}^{\prime}>0$. The proof when $\mathfrak{D}^{\prime}=0$ is similar.

Lemma 22. Suppose $K$ is a function field with $q_{K}=q$ even and $\mathfrak{C}$ is an effective divisor with $\mathfrak{C} \notin 2 \operatorname{Div}(K)$. Suppose $\operatorname{deg}(\mathfrak{C})$ is even. Then

$$
\operatorname{deg}\left(\mathfrak{C}_{\chi}\right) \leq \operatorname{deg}\left(\mathfrak{C}_{1}\right)+g_{K} \# \operatorname{Supp}\left(\mathfrak{C}_{\chi}\right)
$$

Proof. First, since $\chi_{\mathfrak{C}}$ is a character and $L\left(n_{v} v\right) \subseteq \operatorname{ker}\left(\chi_{\mathfrak{C}}\right)$ for all places $v \in M(K)$ by definition, we have

$$
\sum_{v \in \operatorname{Supp}\left(\chi_{\mathfrak{c}}\right)} L\left(n_{v} v\right) \subseteq \operatorname{ker}\left(\chi_{\mathfrak{C}}\right)
$$

Next, $\chi_{\mathfrak{C}}$ is non-trivial by Lemma 17 and $\pi_{\mathbb{A}}(\mathfrak{C}) \subseteq \operatorname{ker}\left(\chi_{\mathfrak{C}}\right)$ as remarked in the proof of Lemma 17 . Thus, the image of $\operatorname{ker}\left(\chi_{\mathfrak{C}}\right)$ under the canonical map $\mathfrak{O}_{\mathbb{A}}(\mathfrak{C}) \mapsto \mathfrak{O}_{\mathbb{A}}(\mathfrak{C}) / \pi_{\mathbb{A}}(\mathfrak{C})$ is a proper subspace. Now by Lemma 10 we have

$$
\operatorname{deg}\left(\mathfrak{C}_{1}^{\mathfrak{c}}\right)=\operatorname{dim}_{\mathbb{F}_{q}} \mathfrak{O}_{\mathbb{A}}(\mathfrak{C}) / \pi_{\mathbb{A}}(\mathfrak{C})>\operatorname{dim}_{\mathbb{F}_{q}}\left(\sum_{v \in \operatorname{Supp}\left(\chi_{\mathfrak{E}}\right)} L\left(n_{v} v\right)\right)
$$

We claim that for any positive integer $m$ and effective divisors $\mathfrak{A}_{1}, \ldots, \mathfrak{A}_{m}$ with $\left(\mathfrak{A}_{i}, \mathfrak{A}_{j}\right)=0$ for all $i \neq j$, we have

$$
\operatorname{dim}_{\mathbb{F}_{q}}\left(\sum_{i=1}^{m} L\left(\mathfrak{A}_{i}\right)\right) \geq \operatorname{deg}\left(\sum_{i=1}^{m} \mathfrak{A}_{i}\right)+1-m g_{K} .
$$

Note that the lemma follows from (14) and (15). We prove (15) by induction on $m$.

The case $m=1$ in (15) follows immediately from the Riemann-Roch Theorem. Now suppose $m>1$. Note that

$$
\left(\sum_{i=1}^{m-1} L\left(\mathfrak{A}_{i}\right)\right) \cap L\left(\mathfrak{A}_{m}\right)=L(0)=\mathbb{F}_{q}
$$


Now by the case $m-1$ of (15) and the Riemann-Roch Theorem we get

$$
\begin{aligned}
\operatorname{dim}_{\mathbb{F}_{q}}\left(\sum_{i=1}^{m} L\left(\mathfrak{A}_{i}\right)\right) & =\operatorname{dim}_{\mathbb{F}_{q}}\left(\sum_{i=1}^{m-1} L\left(\mathfrak{A}_{i}\right)\right)+l\left(\mathfrak{A}_{m}\right)-l(0) \\
& \geq \operatorname{deg}\left(\sum_{i=1}^{m-1} \mathfrak{A}_{i}\right)+1-(m-1) g_{K}+\operatorname{deg}\left(\mathfrak{A}_{m}\right)+1-g_{K}-1 \\
& =\operatorname{deg}\left(\sum_{i=1}^{m} \mathfrak{A}_{i}\right)+1-m g_{K} .
\end{aligned}
$$

Proof of Proposition 6. By Proposition 1,

$$
\sum_{\substack{[F: K]=2 \\ g_{F}=m, q_{F}=q}} \chi(F / \mathfrak{C})=\sum_{\substack{\mathfrak{D}>0 \\ \operatorname{deg}(\mathfrak{D})=m+1-g_{K}}} \sum_{\substack{[F: K]=2, q_{F}=q \\ \operatorname{Disc}_{K}(F)=2 \mathfrak{D}}} \chi(F / \mathfrak{C}) .
$$

Suppose $\operatorname{deg}(\mathfrak{C})$ is even. For all effective divisors $\mathfrak{D}$ with $(\mathfrak{D}, \mathfrak{C})=0, \operatorname{deg}\left(\mathfrak{D}_{2}\right) \geq 2 g_{K}-1$ and $2 \mathfrak{D}_{2} \not \leq \mathfrak{C}_{\chi}$, we have

$$
\sum_{\substack{[F: K]=2, q_{F}=q \\ \operatorname{Disc}_{K}(F)=2 \mathfrak{D}}} \chi(F / \mathfrak{C})=0
$$

by Lemma 19. So suppose either $\operatorname{deg}\left(\mathfrak{D}_{2}\right)<2 g_{K}-1$ or $2 \mathfrak{D}_{2} \leq \mathfrak{C}_{\chi}$. This time we write $\mathfrak{D}=\mathfrak{A}+\mathfrak{B}$ where

$$
\mathfrak{A}=\sum_{v \in \operatorname{Supp}(\mathfrak{D}) \cap \operatorname{Supp}\left(\mathfrak{C}_{\chi}\right)} \operatorname{ord}_{v}(\mathfrak{D}) v, \quad \mathfrak{B}=\sum_{\substack{v \in \operatorname{Supp}(\mathfrak{D}) \\ v \notin \operatorname{Supp}\left(\mathfrak{C}_{\chi}\right)}} \operatorname{ord}_{v}(\mathfrak{D}) v .
$$

Write $\mathfrak{B}=\mathfrak{B}^{\prime}+v_{1}+\cdots+v_{n}$ as in Lemma 21. We note that the places $v_{i}, 1 \leq i \leq n$ are distinct since otherwise we would have $\operatorname{deg}\left(\mathfrak{D}_{2}\right) \geq 2 g_{K}-1$ and $\mathfrak{D}_{2} \not \leq \mathfrak{C}_{\chi}$. Also $n_{v_{i}}=0$ for all $i=1, \ldots, n$ since $\left(\mathfrak{B}, \mathfrak{C}_{\chi}\right)=0$ by construction. Since $\operatorname{deg}(\mathfrak{C})$ is even, Lemma 21 implies that

$$
\sum_{\substack{[F: K]=2, q_{F}=q \\ \operatorname{Disc}_{K}(F)=2 \mathfrak{D}}} \chi(F / \mathfrak{C})= \begin{cases} \pm \sum_{\substack{[F: K]=2, q_{F}=q \\ \operatorname{Disc}_{K}(F)=2 \mathfrak{A}+2 \mathfrak{B}^{\prime}}} \chi(F / \mathfrak{C}) & \text { if } \mathfrak{A}+\mathfrak{B}^{\prime} \neq 0 \\ -2 & \text { if } \mathfrak{A}+\mathfrak{B}^{\prime}=0 .\end{cases}
$$

We next note that for all places $v \in \operatorname{Supp}\left(\mathfrak{B}^{\prime}\right)$ we have $\operatorname{ord}_{v}\left(\mathfrak{B}^{\prime}\right)<2 g_{K}$, since otherwise we would have $\operatorname{deg}\left(\mathfrak{D}_{2}\right) \geq 2 g_{K}-1$ and $\mathfrak{D}_{2} \not \leq \mathfrak{C}_{\chi}$. Thus, if we set

$$
\mathfrak{E}=\sum_{\substack{v \in M(K) \\ \operatorname{deg}(v)<2 g_{K}-1}} 2 g_{K} v
$$


then $\mathfrak{A}+\mathfrak{B}^{\prime} \leq \mathfrak{C}_{\chi}+\mathfrak{E}$.

Now using Lemma 0 together with Lemma 22 and $\operatorname{deg}(\mathfrak{E}) \ll 1$, we see that for all $\epsilon>0$ the number $N$ of effective divisors $\mathfrak{A}+\mathfrak{B}^{\prime} \leq \mathfrak{C}_{\chi}+\mathfrak{E}$ satisfies $N \ll q^{\epsilon \operatorname{deg}\left(\mathfrak{C}_{1}\right)} \leq q^{\epsilon \operatorname{deg}(\mathfrak{C})}$. Here the implicit constant depends only on $\epsilon$ and $K$. Also, we clearly have

$$
\left|\sum_{\substack{[F: K]=2, q_{F}=q \\ \operatorname{Disc}_{K}(F)=2 \mathfrak{A}+2 \mathfrak{B}^{\prime}}} \chi(F / \mathfrak{C})\right| \leq \sum_{\substack{[F: K]=2, q_{F}=q \\ \operatorname{Disc}_{K}(F)=2 \mathfrak{A}+2 \mathfrak{B}^{\prime}}} 1<q^{\operatorname{deg}\left(\mathfrak{A}+\mathfrak{B}^{\prime}\right)}
$$

by Proposition 3. Now by (16)-(19)

$$
\begin{aligned}
& \left|\sum_{\substack{F: K]=2 \\
q_{F}=q, g_{F}=m}} \chi(F / \mathfrak{C})\right| \leq \sum_{i=0}^{m+1-g_{K}} \sum_{\substack{\mathfrak{B} \geq 0 \\
\operatorname{deg}(\mathfrak{B})=i}} \sum_{\substack{[F: K]=2, q_{F}=q \\
\operatorname{sic}\left(\mathfrak{B}_{K}(F)=2 \mathfrak{A}+2 \mathfrak{B}^{\prime} \\
\operatorname{deg}\left(\mathfrak{A}+\mathfrak{B}^{\prime}\right)=m+1-g_{K}-i\right.}} \chi(F / \mathfrak{C}) \mid \\
& \ll \sum_{i=0}^{m+1-g_{K}} \sum_{\substack{\mathfrak{B} \geq 0 \\
\operatorname{deg}(\mathfrak{B})=i}} q^{\epsilon \operatorname{deg}(\mathfrak{C})} q^{m+1-g_{K}-i} \\
& \ll q^{m} q^{\epsilon \operatorname{deg}(\mathfrak{C})} \text {. }
\end{aligned}
$$

Proof of Proposition 7. We first note that the last part of Proposition 7 follows from the first part by setting $\mathfrak{C}=0$. We also note that by Proposition $1, \operatorname{deg}\left(\operatorname{Disc}_{K}(F)\right)=2 m-4 g_{K}+2$ if $g_{F}=m$, $\chi(F / 2 \mathfrak{C})=1$ if $\left(\operatorname{Disc}_{K}(F), \mathfrak{C}\right)=0$ and $\chi(F / 2 \mathfrak{C})=0$ if $\left(\operatorname{Disc}_{K}(F), \mathfrak{C}\right) \neq 0$. It will prove convenient to temporarily set

$$
\chi_{\mathfrak{C}}(\mathfrak{D})= \begin{cases}1 & \text { if }(\mathfrak{C}, \mathfrak{D})=0 \\ 0 & \text { otherwise }\end{cases}
$$

For the case where $q$ is odd set $m^{\prime}=m-2 g_{K}+1$ and choose a place $v_{0} \notin \operatorname{Supp}(\mathfrak{C})$ of odd degree and divisors $\mathfrak{A}_{i, j}$ as in the proof of Proposition 5 above.

For a fixed $i, j$ and $k$,

$$
\sum_{\alpha \in L\left(2 k v_{0}-2 \mathfrak{A}_{i, j}\right)} \chi_{2 \mathfrak{C}}(\alpha)=\#\left\{\alpha \in L\left(2 k v_{0}-2 \mathfrak{A}_{i, j}\right): \operatorname{ord}_{v}(\alpha)=0 \text { all } v \in \operatorname{Supp}(\mathfrak{C})\right\}
$$

For any divisor $\mathfrak{A} \in \operatorname{Div}(K)$ with $\operatorname{Supp}(\mathfrak{A}) \cap \operatorname{Supp}(\mathfrak{C})=\emptyset$, one readily verifies that

$$
\#\left\{\alpha \in L(\mathfrak{A}): \operatorname{ord}_{v}(\alpha)=0 \text { all } v \in \operatorname{Supp}(\mathfrak{C})\right\}=\sum_{0 \leq \mathfrak{D} \leq \mathfrak{C}} \mu(\mathfrak{D}) \# L(\mathfrak{A}-\mathfrak{D})
$$


If $\operatorname{deg}(\mathfrak{A}) \geq 2 g_{K}-1+\operatorname{deg}(\mathfrak{C})$, then

$$
\begin{aligned}
\sum_{0 \leq \mathfrak{D} \leq \mathfrak{C}} \mu(\mathfrak{D}) \# L(\mathfrak{A}-\mathfrak{D}) & =\sum_{\substack{0 \leq \mathfrak{D} \leq \mathfrak{C} \\
\operatorname{deg}(\mathfrak{A})+1-g_{K}}} \mu(\mathfrak{D}) q^{\operatorname{deg}(\mathfrak{A}-\mathfrak{D})+1-g_{K}} \\
& \sum_{0 \leq \mathfrak{D} \leq \mathfrak{C}} \mu(\mathfrak{D}) q^{-\operatorname{deg}(\mathfrak{D})} \\
& =q^{\operatorname{deg}(\mathfrak{A})+1-g_{K}} \prod_{v \in \operatorname{Supp}(\mathfrak{C})}\left(1-q^{-\operatorname{deg}(v)}\right) .
\end{aligned}
$$

If $\operatorname{deg}(\mathfrak{A})<2 g_{K}-1+\operatorname{deg}(\mathfrak{C})$, then

$$
\begin{aligned}
\left|\sum_{0 \leq \mathfrak{D} \leq \mathfrak{C}} \mu(\mathfrak{D})\left(\# L(\mathfrak{A}-\mathfrak{D})-q^{\operatorname{deg}(\mathfrak{A}-\mathfrak{D})+1-g_{K}}\right)\right| & \ll \sum_{\substack{0 \leq \mathfrak{D} \leq \mathfrak{C}\\
}} 1 \\
& \ll q^{\epsilon \operatorname{deg}(\mathfrak{C})}
\end{aligned}
$$

by Lemma 0 and the Riemann-Roch Theorem. Thus, whenever $2 k \operatorname{deg}\left(v_{0}\right)-2 i \geq \operatorname{deg}(\mathfrak{C})+$ $2 \operatorname{deg}\left(v_{0}\right)+2 g_{K}-1$ we have

$$
\sum_{\substack{\alpha \in L\left(2 k v_{0}-2 \mathfrak{A}_{i, j}\right) \\ \alpha \notin L\left(2(k-1) v_{0}-\mathfrak{A}_{i, j}\right)}} \chi_{2 \mathfrak{C}}(\alpha)=q^{2 k \operatorname{deg}\left(v_{0}\right)-2 i+1-g_{K}}\left(1-q^{-2 \operatorname{deg}\left(v_{0}\right)}\right) \prod_{v \in \operatorname{Supp}(\mathfrak{C})}\left(1-q^{-\operatorname{deg}(v)}\right),
$$

and if $2 k \operatorname{deg}\left(v_{0}\right)-2 i<\operatorname{deg}(\mathfrak{C})+2 \operatorname{deg}\left(v_{0}\right)+2 g_{K}-1$

$$
\left|\sum_{\substack{\alpha \in L\left(2 k v_{0}-2 \mathfrak{A}_{i, j}\right) \\ \alpha \notin L\left(2(k-1) v_{0}-\mathfrak{A}_{i, j}\right)}} \chi_{2 \mathfrak{C}}(\alpha)-q^{2 k \operatorname{deg}\left(v_{0}\right)-2 i+1-g_{K}}\left(1-q^{-2 \operatorname{deg}\left(v_{0}\right)}\right) \prod_{v \in \operatorname{Supp}(\mathfrak{C})}\left(1-q^{-\operatorname{deg}(v)}\right)\right|^{\ll q^{\epsilon / 2 \operatorname{deg}(\mathfrak{C})} .}
$$

For notational convenience, temporarily set $c=\operatorname{deg}(\mathfrak{C})+2 \operatorname{deg}\left(v_{0}\right)+2 g_{K}-1$. Combining the above together with Lemma 16 yields

$$
\begin{aligned}
\frac{q-1}{2} \sum_{\substack{[F: K]=2 \\
g_{F}=m, q_{F}=q}} \chi(F / 2 \mathfrak{C}) \\
=J_{K} \sum_{\substack{\left(\mathfrak{B}, \mathfrak{C}+v_{0}\right)=0 \\
\operatorname{deg}(\mathfrak{B}) \leq m^{\prime}}} \mu(\mathfrak{B}) q^{2 m^{\prime}-2 \operatorname{deg}(\mathfrak{B})+1-g_{K}}\left(1-q^{-2 \operatorname{deg}\left(v_{0}\right)}\right) \prod_{v \in \operatorname{Supp}(\mathfrak{C})}\left(1-q^{-\operatorname{deg}(v)}\right) \\
+O\left(\sum_{m^{\prime}-c / 2<n \leq m^{\prime}} q^{\epsilon / 2 \operatorname{deg}(\mathfrak{C})}\left|\sum_{\substack{\left(\mathfrak{B}, \mathfrak{C}+v_{0}\right)=0 \\
\operatorname{deg}(\mathfrak{B})=n}} \mu(\mathfrak{B})\right|\right) .
\end{aligned}
$$


Now via the Euler product and the definition of $L_{K}$,

$$
\begin{aligned}
\sum_{\substack{\mathfrak{B} \geq 0 \\
\left(\mathfrak{B}, \mathfrak{C}+v_{0}\right)=0}} \mu(\mathfrak{B}) q^{-s \operatorname{deg}(\mathfrak{B})} & =\sum_{\mathfrak{B} \geq 0} \mu(\mathfrak{B}) \chi_{\mathfrak{C}+v_{0}}(\mathfrak{B}) q^{-s \operatorname{deg}(\mathfrak{B})} \\
& =\frac{\prod_{v \in M(K)}\left(1-q^{-s \operatorname{deg}(v)}\right)}{\prod_{v \in \operatorname{Supp}\left(\mathfrak{C}+v_{0}\right)}\left(1-q^{-s \operatorname{deg}(v)}\right)} \\
& =\frac{\left(1-q^{-s}\right)\left(1-q^{1-s}\right)}{L_{K}\left(q^{-s}\right)} \prod_{v \in \operatorname{Supp}\left(\mathfrak{C}+v_{0}\right)}\left(1-q^{-s \operatorname{deg}(v)}\right)^{-1} .
\end{aligned}
$$

We note by Lemma 0 that

$$
\left|\prod_{v \in \operatorname{Supp}\left(\mathfrak{C}+v_{0}\right)}\left(1-q^{-s \operatorname{deg}(v)}\right)^{-1}\right| \ll\left|\prod_{v \in \operatorname{Supp}(\mathfrak{C})}\left(1-q^{-s \operatorname{deg}(v)}\right)^{-1}\right| \ll q^{\epsilon / 2 \operatorname{deg}(\mathfrak{C})}
$$

for all $s \in \mathbb{C}$ with $\Re(s) \geq 1 / 2$.

Combining (21) and (22) yields

$$
\left|\sum_{\substack{\left(\mathfrak{B}, \mathfrak{C}+v_{0}\right)=0 \\ \operatorname{deg}(\mathfrak{B})=n}} \mu(\mathfrak{B})\right| \ll q^{(1 / 2+\epsilon) n} q^{\epsilon / 2 \operatorname{deg}(\mathfrak{C})}
$$

for all $n \geq 0$. Also by (21) (with $s=2$ ) and (22), we have

$$
\begin{gathered}
\sum_{\substack{\left(\mathfrak{B}, \mathfrak{C}+v_{0}\right)=0 \\
\operatorname{deg}(\mathfrak{B}) \leq m^{\prime}}} \mu(\mathfrak{B}) q^{-2 \operatorname{deg}(\mathfrak{B})}=\zeta_{K}(2)^{-1}\left(1-q^{-2 \operatorname{deg}\left(v_{0}\right)}\right)^{-1} \\
\times \prod_{v \in \operatorname{Supp}(\mathfrak{C})}\left(1-q^{-\operatorname{deg}(v)}\right)^{-1}\left(1+q^{-\operatorname{deg}(v)}\right)^{-1} \\
+O\left(q^{(-3 / 2+\epsilon) m^{\prime}} q^{\epsilon \operatorname{deg}(\mathfrak{C})}\right)
\end{gathered}
$$

The case where $q$ is odd follows from (20), (23) and (24).

We now turn to the case of even characteristic. For $s \in \mathbb{C}$ with $\Re(s)>1$ we set

$$
\begin{aligned}
f(s) & =\sum_{\mathfrak{D} \geq 0} q^{-(s+1) \operatorname{deg}(\mathfrak{D})} \phi(\mathfrak{D}) \chi_{\mathfrak{C}}(\mathfrak{D}) \\
& =\sum_{\mathfrak{D} \geq 0} q^{-s \operatorname{deg}(\mathfrak{D})} q^{-\operatorname{deg}(\mathfrak{D})} \phi(\mathfrak{D}) \chi_{\mathfrak{C}}(\mathfrak{D}) .
\end{aligned}
$$

Since $q^{-\operatorname{deg}(\mathfrak{A}+\mathfrak{B})} \phi(\mathfrak{A}+\mathfrak{B}) \chi_{\mathfrak{C}}(\mathfrak{A}+\mathfrak{B})=q^{-\operatorname{deg}(\mathfrak{A})} \phi(\mathfrak{A}) \chi_{\mathfrak{C}}(\mathfrak{A}) q^{-\operatorname{deg}(\mathfrak{B})} \phi(\mathfrak{B}) \chi_{\mathfrak{C}}(\mathfrak{B})$ whenever $(\mathfrak{A}, \mathfrak{B})=$ 
0 , we have

$$
\begin{aligned}
f(s) & =\prod_{v \in M(K)}\left(1+q^{-\operatorname{deg}(v)} \phi(v) \chi_{\mathfrak{C}}(v) q^{-s \operatorname{deg}(v)}+q^{-2 \operatorname{deg}(v)} \phi(2 v) \chi_{\mathfrak{C}}(2 v) q^{-2 s \operatorname{deg}(v)}+\cdots\right) \\
& =\frac{\prod_{v \in M(K)}\left(1+\left(1-q^{-\operatorname{deg}(v)}\right) q^{-s \operatorname{deg}(v)}+\left(q^{\operatorname{deg}(v)}-1\right) q^{-2 s \operatorname{deg}(v)}+\cdots\right)}{\prod_{v \in \operatorname{Supp}(\mathfrak{C})}\left(1+\left(1-q^{-\operatorname{deg}(v)}\right) q^{-s \operatorname{deg}(v)}+\left(q^{\operatorname{deg}(v)}-1\right) q^{-2 s \operatorname{deg}(v)}+\cdots\right)} \\
& =\frac{\prod_{v \in M(K)}\left(1-q^{-s \operatorname{deg}(v)}\right)^{-1}\left(1-q^{-(s+1) \operatorname{deg}(v)}\right)}{\prod_{v \in \operatorname{Supp}(\mathfrak{C})}\left(1-q^{-s \operatorname{deg}(v)}\right)^{-1}\left(1-q^{-(s+1) \operatorname{deg}(v))}\right.} \\
& =\frac{\zeta_{K}(s)}{\zeta_{K}(s+1)} \prod_{v \in \operatorname{Supp}(\mathfrak{C})}\left(1-q^{-s \operatorname{deg}(v)}\right)\left(1-q^{-(s+1) \operatorname{deg}(v)}\right)^{-1}
\end{aligned}
$$

We see that $f(s)$ is holomorphic on any set $\{s \in \mathbb{C}: \Re(s) \geq \epsilon-1 / 2,-\pi / \log q \leq \Im(s)<\pi / \log q\}$ except for a simple pole at $s=1$.

Using a standard Tauberian argument (see [8, Theorem 17.1], for example) we have

$$
\sum_{\substack{\mathfrak{D} \geq 0 \\ \operatorname{deg}(\mathfrak{D})=j}} q^{-j} \phi(\mathfrak{D}) \chi_{\mathfrak{C}}(\mathfrak{D})=r \log (q) q^{j}+O\left(M q^{(\epsilon-1) j}\right)
$$

where $r$ is the residue of $f(s)$ at $s=1, M$ is the maximum of $|f(s)|$ on the set of $s$ with $\Re(s)=\epsilon-1 / 2$, and the implicit constant is absolute. Since $\zeta_{\mathbb{F}_{q}(X)}=\frac{1}{\left(1-q^{-s}\right)\left(1-q^{1-s}\right)}$, we see that

$$
\operatorname{Res}_{s=1} \zeta_{\mathbb{F}_{q}(X)}(s)=\frac{q}{(q-1) \log (q)}
$$

Therefore

$$
\begin{aligned}
r & =\operatorname{Res}_{s=1} f(s) \\
& =\frac{L_{K}\left(q^{-1}\right)}{\zeta_{K}(2)} \prod_{v \in \operatorname{Supp}(\mathfrak{C})}\left(1+q^{-\operatorname{deg}(v)}\right)^{-1} \operatorname{Res}_{s=1} \zeta_{\mathbb{F}_{q}(X)}(s) \\
& =\frac{L_{K}\left(q^{-1}\right)}{\zeta_{K}(2)} \prod_{v \in \operatorname{Supp}(\mathfrak{C})}\left(1+q^{-\operatorname{deg}(v)}\right)^{-1} \frac{q}{(q-1) \log (q)} \\
& =\frac{J_{K} q^{1-g_{K}}}{\zeta_{K}(2)(q-1) \log (q)} \prod_{v \in \operatorname{Supp}(\mathfrak{C})}\left(1+q^{-\operatorname{deg}(v)}\right)^{-1} .
\end{aligned}
$$


Using Lemma 0, we get

$$
\begin{aligned}
M & =\max _{\Re(s)=\epsilon-1 / 2}\left\{\left|\prod_{v \notin \operatorname{Supp}(\mathfrak{C})}\left(1-q^{-s \operatorname{deg}(v)}\right)^{-1}\left(1-q^{-(s+1) \operatorname{deg}(v)}\right)\right|\right\} \\
& \ll \max _{\Re(s)=\epsilon-1 / 2}\left\{\left|\prod_{v \in \operatorname{Supp}(\mathfrak{C})}\left(1-q^{-s \operatorname{deg}(v)}\right)\left(1-q^{-(s+1) \operatorname{deg}(v)}\right)^{-1}\right|\right\} \\
& \leq \prod_{v \in \operatorname{Supp}(\mathfrak{C})}\left(1+q^{-\epsilon+1 / 2)}\right)\left(1-q^{-\epsilon-1 / 2}\right)^{-1} \\
& <\prod_{v \in \operatorname{Supp}(\mathfrak{c})}\left(1+q^{1 / 2}\right)\left(1-q^{-1 / 2}\right)^{-1} \\
& \ll q^{\epsilon \operatorname{deg}(\mathfrak{C})} .
\end{aligned}
$$

We now set $j=m-2 g_{K}+1$ in (25) and multiply through by $q^{j}$. Using (26) and (27) gives

$$
\sum_{\substack{\mathfrak{D} \geq 0 \\ \operatorname{deg}(\mathfrak{D})=m-2 g_{K}+1}} \phi(\mathfrak{D}) \chi_{\mathfrak{C}}(\mathfrak{D})=\frac{J_{K} q^{3-5 g_{K}}}{\zeta_{K}(2)(q-1)} q^{2 m} \prod_{v \in \operatorname{Supp}(\mathfrak{C})}\left(1+q^{-\operatorname{deg}(v)}\right)^{-1}+O\left(q^{\epsilon \operatorname{deg}(\mathfrak{C})} q^{\epsilon m}\right)
$$

Using this estimate together with the Corollary to Proposition 3 and (0), we get

$$
\begin{aligned}
& \sum_{\substack{\mathfrak{D} \in \operatorname{Div}(K) \\
\mathfrak{D} \geq 0}} \sum_{\substack{[F: K]=2, q_{F}=q \\
\operatorname{Disc}_{K}(F)=2 \mathfrak{D}}} \chi(F / 2 \mathfrak{C}) \\
& =\sum_{\substack{\mathfrak{D} \in \operatorname{Div}(K) \\
\mathfrak{D} \geq 0 \\
\operatorname{deg}(\mathfrak{D})=m}} N(\mathfrak{D}) \chi_{\mathfrak{C}}(\mathfrak{D}) \\
& =\sum_{\substack{\mathfrak{D} \in \operatorname{Div}(K) \\
\mathfrak{D} \geq 0 \\
\operatorname{deg}(\mathfrak{D})=m-2 g_{K}+1}} 2 \phi(\mathfrak{D}) \chi_{\mathfrak{C}}(\mathfrak{D})+O\left(\sum_{\substack{\mathfrak{D} \in \operatorname{Div}(K) \\
\mathfrak{D} \geq 0 \\
\operatorname{deg}(\mathfrak{D})=m-2 g_{K}+1}} \chi_{\mathfrak{C}}(\mathfrak{D})\right) \\
& =\frac{2 J_{K} q^{3-5 g_{K}}}{\zeta_{K}(2)(q-1)} q^{2 m} \prod_{v \in \operatorname{Supp}(\mathfrak{C})}\left(1+q^{-\operatorname{deg}(v)}\right)^{-1}+O\left(q^{\epsilon m} q^{\epsilon \operatorname{deg}(\mathfrak{C})}+q^{m}\right) .
\end{aligned}
$$

We remark that one can use a similar Tauberian argument for the case where $q$ is odd (cf. [8, Proposition 17.2] which is the case where $\mathfrak{C}=0$ ), though this leads to an estimate for the number of all square-free effective divisors $\mathfrak{D}$ of fixed degree with $(\mathfrak{C}, \mathfrak{D})=0$. This gives Proposition 7 if the map $\psi$ in Proposition 2 has a trivial kernel, i.e., if $J_{K}$ is odd. 


\section{Proofs of Theorems 1, 2 And 3}

From the definitions in $\S 1$ we see that

$$
\sum_{\substack{[F: K]=2 \\
g F \\
g_{F}=m, q_{F}=q_{K}}} L_{F}^{*}\left(q^{-s}\right) L_{F}^{*}\left(q^{-t}\right)=\sum_{\substack { \mathfrak{C} \geq 0 \\
\begin{subarray}{c}{\mathfrak{A}, \mathfrak{B} \geq 0 \\
\mathfrak{A}+\mathfrak{B}=\mathfrak{C}{ \mathfrak { C } \geq 0 \\
\begin{subarray} { c } { \mathfrak { A } , \mathfrak { B } \geq 0 \\
\mathfrak { A } + \mathfrak { B } = \mathfrak { C } } }\end{subarray}} q^{-s \operatorname{deg}(\mathfrak{A})} q^{-t \operatorname{deg}(\mathfrak{B})} \sum_{\substack{[F: K]=2 \\
g_{F}=m, q_{F}=q_{K}}} \chi(F /(\mathfrak{A}+\mathfrak{B})),
$$

with similar expressions including Möbius functions for sums involving quotients of $L$-functions. As indicated at the end of $\S 1$ and demonstrated in Propositions 5-7, the summands above where $\mathfrak{C}=\mathfrak{A}+\mathfrak{B} \in 2 \operatorname{Div}(K)$ dominate. We will deal with these summands first.

Lemma 23. Suppose $K$ is a function field with field of constants $\mathbb{F}_{q}$ and $s, t \in \mathbb{C}$ with $\Re(t), \Re(s)>$ 1/2. Then

$$
\begin{aligned}
& \sum_{\substack { \mathfrak{C} \geq 0 \\
\begin{subarray}{c}{\mathfrak{A}, \mathfrak{B} \geq 0 \\
\mathfrak{A}+\mathfrak{B}=2 \mathfrak{C}{ \mathfrak { C } \geq 0 \\
\begin{subarray} { c } { \mathfrak { A } , \mathfrak { B } \geq 0 \\
\mathfrak { A } + \mathfrak { B } = 2 \mathfrak { C } } }\end{subarray}} q^{-s \operatorname{deg}(\mathfrak{A})} q^{-t \operatorname{deg}(\mathfrak{B})} \prod_{v \in \operatorname{Supp}(\mathfrak{C})}\left(1+q^{-\operatorname{deg}(v)}\right)^{-1}=\zeta_{K}(2) \zeta_{K}(2 s) \zeta_{K}(2 t) \sigma_{1}(s, t), \\
& \sum_{\substack { \mathfrak{C} \geq 0 \\
\begin{subarray}{c}{\mathfrak{A}, \mathfrak{B} \geq 0 \\
\mathfrak{A}+\mathfrak{B}=2 \mathfrak{C}{ \mathfrak { C } \geq 0 \\
\begin{subarray} { c } { \mathfrak { A } , \mathfrak { B } \geq 0 \\
\mathfrak { A } + \mathfrak { B } = 2 \mathfrak { C } } }\end{subarray}} \mu(\mathfrak{B}) q^{-s \operatorname{deg}(\mathfrak{A})} q^{-t \operatorname{deg}(\mathfrak{B})} \prod_{v \in \operatorname{Supp}(\mathfrak{C})}\left(1+q^{-\operatorname{deg}(v)}\right)^{-1}=\zeta_{K}(2) \zeta_{K}(2 s) \sigma_{2}(s, t),
\end{aligned}
$$

and

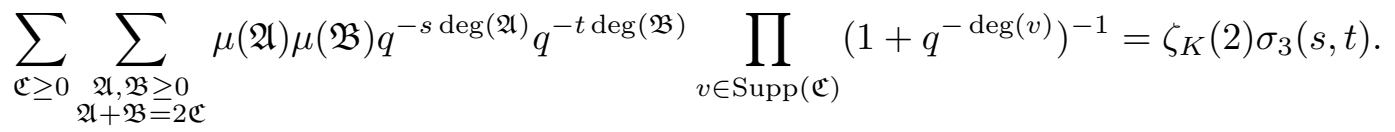

Proof. If $\mathfrak{A}+\mathfrak{B}=2 \mathfrak{C}$, then $-t \operatorname{deg}(\mathfrak{B})-s \operatorname{deg}(\mathfrak{A})=-(t-s) \operatorname{deg}(\mathfrak{B})-2 s \operatorname{deg}(\mathfrak{C})$. Thus

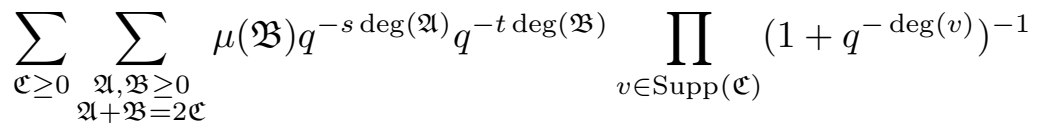

$$
\begin{aligned}
& =\sum_{\mathfrak{C} \geq 0} \sum_{0 \leq \mathfrak{B} \leq 2 \mathfrak{C}} \mu(\mathfrak{B}) q^{-(t-s) \operatorname{deg}(\mathfrak{B})} q^{-2 s \operatorname{deg}(\mathfrak{C})} \prod_{v \in \operatorname{Supp}(\mathfrak{C})}\left(1+q^{-\operatorname{deg}(v)}\right)^{-1}
\end{aligned}
$$

and similarly for sums where the $\mu(\mathfrak{B})$ term is absent. If $\mathfrak{A}+\mathfrak{B}=2 \mathfrak{C}$ and $\mu(\mathfrak{A}) \mu(\mathfrak{B}) \neq 0$, then $\mathfrak{A}=\mathfrak{B}=\mathfrak{C}$ and $\mu(\mathfrak{A}) \mu(\mathfrak{B})=|\mu(\mathfrak{C})|$. Set

$$
\begin{aligned}
& \theta_{1}(\mathfrak{C})=q^{-2 s \operatorname{deg}(\mathfrak{C})} \prod_{v \in \operatorname{Supp}(\mathfrak{C})}\left(1+q^{-\operatorname{deg}(v)}\right)^{-1} \sum_{0 \leq \mathfrak{B} \leq 2 \mathfrak{C}} q^{-(t-s) \operatorname{deg}(\mathfrak{B})} \\
& \theta_{2}(\mathfrak{C})=q^{-2 s \operatorname{deg}(\mathfrak{C})} \prod_{v \in \operatorname{Supp}(\mathfrak{C})}\left(1+q^{-\operatorname{deg}(v)}\right)^{-1} \sum_{0 \leq \mathfrak{B} \leq 2 \mathfrak{C}} \mu(\mathfrak{B}) q^{-(t-s) \operatorname{deg}(\mathfrak{B})} \\
& \theta_{3}(\mathfrak{C})=|\mu(\mathfrak{C})| q^{-(s+t) \operatorname{deg}(\mathfrak{C})} \prod_{v \in \operatorname{Supp}(\mathfrak{C})}\left(1+q^{-\operatorname{deg}(v)}\right)^{-1}
\end{aligned}
$$


Note that $\theta_{i}(\mathfrak{C}+\mathfrak{D})=\theta_{i}(\mathfrak{C}) \theta_{i}(\mathfrak{D})$ whenever $(\mathfrak{C}, \mathfrak{D})=0$ for all $i$. Thus

$$
\sum_{\mathfrak{C} \geq 0} \theta_{i}(\mathfrak{C})=\prod_{v \in M(K)}\left(1+\sum_{k=1}^{\infty} \theta_{i}(k v)\right) .
$$

Suppose that $s \neq t$. Then for all positive integers $k$ and all places $v$

$$
\begin{aligned}
\theta_{1}(k v) & =\left(1+q^{-\operatorname{deg}(v)}\right)^{-1} q^{-2 k s \operatorname{deg}(v)} \sum_{i=0}^{2 k} q^{-(t-s) i \operatorname{deg}(v)} \\
& =\left(1+q^{-\operatorname{deg}(v)}\right)^{-1} q^{-2 k s \operatorname{deg}(v)}\left(1-q^{-(t-s)(2 k+1) \operatorname{deg}(v)}\right)\left(1-q^{-(t-s) \operatorname{deg}(v)}\right)^{-1} \\
& =\left(1+q^{-\operatorname{deg}(v)}\right)^{-1}\left(q^{-(2 k+1) s \operatorname{deg}(v)}-q^{-(2 k+1) t \operatorname{deg}(v)}\right)\left(q^{-s \operatorname{deg}(v)}-q^{-t \operatorname{deg}(v)}\right)^{-1} .
\end{aligned}
$$

Therefore

$$
\begin{aligned}
\sum_{k=1}^{\infty} \theta_{1}(k v)=(1 & \left.+q^{-\operatorname{deg}(v)}\right)^{-1}\left(q^{-s \operatorname{deg}(v)}-q^{-t \operatorname{deg}(v)}\right)^{-1} \\
& \times\left(q^{-3 s \operatorname{deg}(v)}\left(1-q^{-2 s \operatorname{deg}(v)}\right)^{-1}-q^{-3 t \operatorname{deg}(v)}\left(1-q^{-2 t \operatorname{deg}(v)}\right)^{-1}\right) \\
= & \left(1+q^{-\operatorname{deg}(v)}\right)^{-1}\left(1-q^{-2 s \operatorname{deg}(v)}\right)^{-1}\left(1-q^{-2 t \operatorname{deg}(v)}\right)^{-1}\left(q^{-s \operatorname{deg}(v)}-q^{-t \operatorname{deg}(v)}\right) \\
& \times\left(q^{-3 s \operatorname{deg}(v)}-q^{-3 t \operatorname{deg}(v)}-q^{-2(s+t) \operatorname{deg}(v)}\left(q^{-s \operatorname{deg}(v)}-q^{-t \operatorname{deg}(v)}\right)\right. \\
=(1 & \left.-q^{-2 \operatorname{deg}(v)}\right)^{-1}\left(1-q^{-2 s \operatorname{deg}(v)}\right)^{-1}\left(1-q^{-2 t \operatorname{deg}(v)}\right)^{-1}\left(1-q^{-\operatorname{deg}(v)}\right) \\
& \times\left(q^{-2 s \operatorname{deg}(v)}+q^{-(s+t) \operatorname{deg}(v)}+q^{-2 t \operatorname{deg}(v)}-q^{-2(s+t) \operatorname{deg}(v)}\right)
\end{aligned}
$$

so that by $(29)$

$$
\begin{aligned}
\sum_{\mathfrak{C} \geq 0} \theta_{1}(\mathfrak{C})= & \zeta_{K}(2) \zeta_{K}(2 s) \zeta_{K}(2 t) \\
\times & \prod_{v \in M(K)}\left(\left(1-q^{-2 \operatorname{deg}(v)}\right)\left(1-q^{-2 s \operatorname{deg}(v)}\right)\left(1-q^{-2 t \operatorname{deg}(v)}\right)\right. \\
& \left.\quad+\left(1-q^{-\operatorname{deg}(v)}\right)\left(q^{-2 s \operatorname{deg}(v)}+q^{-(s+t) \operatorname{deg}(v)}+q^{-2 t \operatorname{deg}(v)}-q^{-2(s+t) \operatorname{deg}(v)}\right)\right) \\
= & \zeta_{K}(2) \zeta_{K}(2 s) \zeta_{K}(2 t) \sigma_{1}(s, t) .
\end{aligned}
$$

Now if $s=t$, then via term-by-term differentiation of

$$
\sum_{k=1}^{\infty} x^{2 k+1}=x \sum_{k=1}^{\infty} x^{2 k}=x^{3} \sum_{k=0}^{\infty} x^{2 k}=\frac{x^{3}}{1-x^{2}}
$$

shows that

$$
\begin{aligned}
\sum_{k=1}^{\infty} \theta_{1}(k v) & =\left(1+q^{-\operatorname{deg}(v)}\right)^{-1} \sum_{k=1}^{\infty}(2 k+1) q^{-2 s k \operatorname{deg}(v)} \\
& =\left(1+q^{-\operatorname{deg}(v)}\right)^{-1} \frac{3 q^{-2 s \operatorname{deg}(v)}-q^{-4 s \operatorname{deg}(v)}}{\left(1-q^{-2 s \operatorname{deg}(v)}\right)^{2}} \\
& =\left(1-q^{-2 \operatorname{deg}(v)}\right)^{-1}\left(1-q^{-2 s \operatorname{deg}(v)}\right)^{-2}\left(1-q^{-\operatorname{deg}(v)}\right)\left(3 q^{-2 s \operatorname{deg}(v)}-q^{-4 s \operatorname{deg}(v)}\right) .
\end{aligned}
$$


Using this together with (29) yields

$$
\begin{aligned}
\sum_{\mathfrak{C} \geq 0} \theta_{1}(\mathfrak{C}) & =\zeta_{K}(2) \zeta_{K}(2 s)^{2} \\
& \times \prod_{v \in M(K)}\left(1-q^{-2 \operatorname{deg}(v)}\right)\left(1-q^{-2 s \operatorname{deg}(v)}\right)^{2}+\left(1-q^{-\operatorname{deg}(v)}\right)\left(3 q^{-2 s \operatorname{deg}(v)}-q^{-4 s \operatorname{deg}(v)}\right) \\
& =\zeta_{K}(2) \zeta_{K}(2 s) \zeta_{K}(2 t) \sigma_{1}(s, t)
\end{aligned}
$$

when $s=t$. Together, (28) and (30) (when $s \neq t$ ), or (28) and (30') (when $s=t$ ) proves the first part of the lemma.

For all positive integers $k$ and $v \in M(K)$ we have

$$
\theta_{2}(k v)=q^{-2 s k \operatorname{deg}(v)}\left(1+q^{-\operatorname{deg}(v)}\right)^{-1}\left(1-q^{-(t-s) \operatorname{deg}(v)}\right),
$$

so that

$$
\begin{aligned}
\sum_{k=1}^{\infty} \theta(k v) & =\left(1+q^{-\operatorname{deg}(v)}\right)^{-1}\left(1-q^{-(t-s) \operatorname{deg}(v)}\right) q^{-2 s \operatorname{deg}(v)}\left(1-q^{-2 s \operatorname{deg}(v)}\right)^{-1} \\
& =\left(1-q^{-2 \operatorname{deg}(v)}\right)^{-1}\left(1-q^{-2 s \operatorname{deg}(v)}\right)^{-1}\left(1-q^{-\operatorname{deg}(v)}\right)\left(q^{-2 s \operatorname{deg}(v)}-q^{-(t+s) \operatorname{deg}(v)}\right) .
\end{aligned}
$$

Whence by (29)

$$
\begin{aligned}
\sum_{\mathfrak{C} \geq 0} \theta_{2}(\mathfrak{C})= & \zeta_{K}(2) \zeta_{K}(2 s) \\
\times & \prod_{v \in M(K)}\left(\left(1-q^{-2 \operatorname{deg}(v)}\right)\left(1-q^{-2 s \operatorname{deg}(v)}\right)\right. \\
& \left.\quad+\left(1-q^{-\operatorname{deg}(v)}\right)\left(q^{-2 s \operatorname{deg}(v)}-q^{-(t+s) \operatorname{deg}(v)}\right)\right) \\
= & \zeta_{K}(2) \zeta_{K}(2 s) \sigma_{2}(s, t) .
\end{aligned}
$$

The second part of Lemma 23 follows from (28) and (31).

Finally, we easily see that for all positive integers $k$ and places $v$

$$
\theta_{3}(k v)= \begin{cases}q^{-(s+t) \operatorname{deg}(v)}\left(1+q^{-\operatorname{deg}(v)}\right)^{-1} & \text { if } k=1 \\ 0 & \text { if } k>1 .\end{cases}
$$

Thus

$$
\begin{aligned}
\prod_{v \in M(K)}\left(1+\sum_{k=1}^{\infty} \theta_{3}(k v)\right) & =\prod_{v \in M(K)} 1+q^{-(s+t) \operatorname{deg}(v)}\left(1+q^{-\operatorname{deg}(v)}\right)^{-1} \\
& =\prod_{v \in M(K)}\left(1+q^{-\operatorname{deg}(v)}\right)^{-1}\left(1+q^{-\operatorname{deg}(v)}+q^{-(s+t) \operatorname{deg}(v)}\right) \\
& =\prod_{v \in M(K)}\left(1-q^{-2 \operatorname{deg}(v)}\right)^{-1}\left(1-q^{-\operatorname{deg}(v)}\right)\left(1+q^{-\operatorname{deg}(v)}+q^{-(s+t) \operatorname{deg}(v)}\right) \\
& =\zeta_{K}(2) \sigma_{3}(s, t) .
\end{aligned}
$$

The last part of the lemma follows from this and (29). 
Lemma 24. Let $K$ be a function field with field of constants $\mathbb{F}_{q}$. Suppose $m$ is a positive integer and $\epsilon>0$. Then for all $s, t \in \mathbb{C}$ with $\Re(t), \Re(s)>1 / 2+\epsilon$

$$
\begin{gathered}
\sum_{\substack { \mathfrak{C} \geq 0 \\
\begin{subarray}{c}{\mathfrak{A}, \mathfrak{B} \in \operatorname{Div}(K) \\
\mathfrak{A}, \mathfrak{B} \geq 0 \\
\mathfrak{A}+\mathfrak{B}=2 \mathfrak{C}{ \mathfrak { C } \geq 0 \\
\begin{subarray} { c } { \mathfrak { A } , \mathfrak { B } \in \operatorname { D i v } ( K ) \\
\mathfrak { A } , \mathfrak { B } \geq 0 \\
\mathfrak { A } + \mathfrak { B } = 2 \mathfrak { C } } }\end{subarray}} q^{-s \operatorname{deg}(\mathfrak{A})} q^{-t \operatorname{deg}(\mathfrak{B})} \sum_{\substack{[F: K]=2 \\
g_{F}=m, q_{F}=q}} \chi(F / 2 \mathfrak{C}) \\
=\frac{2 J_{K} q^{3-5 g_{K}} \zeta_{K}(2 s) \zeta_{K}(2 t)}{q-1} \sigma_{1}(s, t) q^{2 m}+ \begin{cases}O\left(q^{(1 / 2+\epsilon) m}\right) & \text { if } q \text { is odd, } \\
O\left(q^{m}\right) & \text { if } q \text { is even, }\end{cases} \\
\sum_{\substack{\mathfrak{C} \geq 0 \\
\mathfrak{A}, \mathfrak{B} \in \operatorname{Div}(K) \\
\mathfrak{A}, \mathfrak{B} \geq 0}} \mu(\mathfrak{B}) q^{-s \operatorname{deg}(\mathfrak{A})} q^{-t \operatorname{deg}(\mathfrak{B})} \sum_{\substack{[F: K]=2 \\
g_{F}=m, q_{F}=q}} \chi\left(F / 2 \mathfrak{C}^{\mathfrak{C}}\right) \\
=\frac{2 J_{K} q^{3-5 g_{K} \zeta_{K}(2 s)}}{q-1} \sigma_{2}(s, t) q^{2 m}+ \begin{cases}O\left(q^{(1 / 2+\epsilon) m}\right) & \text { if } q \text { is odd, } \\
O\left(q^{m}\right) & \text { if } q \text { is even, }\end{cases}
\end{gathered}
$$

and

$$
\begin{aligned}
\sum_{\substack { \mathfrak{C} \geq 0 \\
\begin{subarray}{c}{\mathfrak{A}, \mathfrak{B} \in \operatorname{Div}(K) \\
\mathfrak{A}, \mathfrak{B} \geq 0 \\
\mathfrak{A}+\mathfrak{B}=2 \mathfrak{C}{ \mathfrak { C } \geq 0 \\
\begin{subarray} { c } { \mathfrak { A } , \mathfrak { B } \in \operatorname { D i v } ( K ) \\
\mathfrak { A } , \mathfrak { B } \geq 0 \\
\mathfrak { A } + \mathfrak { B } = 2 \mathfrak { C } } }\end{subarray}} \mu(\mathfrak{A}) \mu(\mathfrak{B}) q^{-s \operatorname{deg}(\mathfrak{A})} q^{-t \operatorname{deg}(\mathfrak{B})} \sum_{\substack{[F: K]=2 \\
g_{F}=m, q_{F}=q}} \chi(F / 2 \mathfrak{C}) \\
=\frac{2 J_{K} q^{3-5 g_{K}}}{q-1} \sigma_{3}(s, t) q^{2 m}+ \begin{cases}O\left(q^{(1 / 2+\epsilon) m}\right) & \text { if } q \text { is odd, } \\
O\left(q^{m}\right) & \text { if } q \text { is even, }\end{cases}
\end{aligned}
$$

where the implicit constants depend only on $K$ and $\epsilon$.

Proof. The main terms follow directly from Proposition 7 and Lemma 23. As for the sums over the error terms in Proposition 7 , let $\mathfrak{m}=\min \{\Re(s), \Re(t)\}$. Then for all $\delta>0$ we have

$$
\begin{aligned}
\sum_{\mathfrak{C} \geq 0} \sum_{\substack{\mathfrak{A}, \mathfrak{B} \geq 0 \\
\mathfrak{A}+\mathfrak{B}=2 \mathfrak{C}}} q^{-\Re(s) \operatorname{deg}(\mathfrak{A})} q^{-\Re(t) \operatorname{deg}(\mathfrak{B})} & \leq \sum_{\mathfrak{C} \geq 0} q^{-2 \mathfrak{m} \operatorname{deg}(\mathfrak{C})} \sum_{0 \leq \mathfrak{D} \leq 2 \mathfrak{C}} 1 \\
& \ll \sum_{\mathfrak{C} \geq 0} q^{-(2 \mathfrak{m}-\delta / 2) \operatorname{deg}(\mathfrak{C})},
\end{aligned}
$$

by Lemma 0 , where the implicit constant depends only on $K$ and $\delta$. Now set $2 \delta=\mathfrak{m}-1 / 2$. Then by (32) and replacing the $\epsilon$ in Proposition 7 with $\delta / 2$, we have

$$
\begin{aligned}
& \sum_{\substack { \mathfrak{C} \geq 0 \\
\begin{subarray}{c}{\mathfrak{A}, \mathfrak{B} \geq 0 \\
\mathfrak{A}+\mathfrak{B}=2 \mathfrak{C}{ \mathfrak { C } \geq 0 \\
\begin{subarray} { c } { \mathfrak { A } , \mathfrak { B } \geq 0 \\
\mathfrak { A } + \mathfrak { B } = 2 \mathfrak { C } } }\end{subarray}} q^{-\Re(s) \operatorname{deg}(\mathfrak{A})} q^{-\Re(t) \operatorname{deg}(\mathfrak{B})} q^{(\delta / 2) \operatorname{deg}(\mathfrak{C})} \ll \sum_{\mathfrak{C} \geq 0} q^{-(2 \mathfrak{m}-\delta) \operatorname{deg}(\mathfrak{C})} \\
& \ll 1 \text {. }
\end{aligned}
$$

The error terms in Lemma 24 follow from Proposition 7 and (33). 
With the sums over the main terms done, we now turn to the sums over the error terms, i.e., the sums where $\mathfrak{A}+\mathfrak{B} \notin 2 \operatorname{Div}(K)$. When no Möbius function appears with a given divisor, then we can use the following estimate when the degree of that divisor is relatively large in terms of the parameter $m$.

Lemma 25. Suppose $K$ is a function field with field of constants $\mathbb{F}_{q}$ and $m$ is a non-negative integer. Let $\epsilon>0$ and suppose $s, t \in \mathbb{C}$ with $\Re(t), \Re(s)>1 / 2+\epsilon$. Then for any quadratic extension $F \supset K$ with $g_{F}=m$ and $q_{F}=q$

$$
\left.\sum_{\mathfrak{B} \geq 0} q^{-\Re(t) \operatorname{deg}(\mathfrak{B})} \mid \sum_{\substack{\mathfrak{A} \geq 0 \mathfrak{A}+\mathfrak{B} \notin 2 \operatorname{Div}(K) \\ \operatorname{deg}(\mathfrak{A})>2 m-2 g_{K}}} q^{-s \operatorname{deg}(\mathfrak{A})} \chi(F / \mathfrak{A})\right) \mid \ll q^{-2 m(\Re(s)-1 / 2)},
$$

where the implicit constant depends only on $K$ and $\epsilon$.

Proof. Fix a quadratic extension $F$ of $K$ with $g_{F}=m$ and $q_{F}=q$. For the moment, fix a divisor $\mathfrak{B} \geq 0$ and write $\mathfrak{B}=\mathfrak{B}^{\prime}+2 \mathfrak{B}^{\prime \prime}$ where $\mathfrak{B}^{\prime}, \mathfrak{B}^{\prime \prime} \geq 0$ and $\mathfrak{B}^{\prime}$ is square-free. Let $n>2 m-2 g_{K}$ and suppose that $\mathfrak{A}$ is an effective divisor such that $\operatorname{deg}(\mathfrak{A})=n$ and $\mathfrak{A}+\mathfrak{B}=2 \mathfrak{C}$ for some divisor $\mathfrak{C}$. Then we must have $\mathfrak{A}=\mathfrak{B}^{\prime}+2 \mathfrak{C}^{\prime}$ for some effective divisor $\mathfrak{C}^{\prime}$ with $2 \operatorname{deg}\left(\mathfrak{C}^{\prime}\right)=n-\operatorname{deg}\left(\mathfrak{B}^{\prime}\right)$. By a theorem of Weil (see [8, Theorem 9.16B]), $L_{F}^{*}\left(q^{-s}\right)$ is a polynomial of degree $2 g_{F}-2 g_{K}$ in $q^{-s}$. Since $n>2 m-2 g_{K}=2 g_{F}-2 g_{K}$,

$$
\begin{aligned}
0 & =\sum_{\substack{\mathfrak{A} \geq 0 \\
\operatorname{deg}(\mathfrak{A})=n}} \chi(F / \mathfrak{A}) \\
& =\sum_{\substack{\mathfrak{A} \geq 0 \\
\operatorname{deg}(\mathfrak{A})=n \\
\mathfrak{A}=\mathfrak{B}^{\prime}+2 \mathfrak{C}^{\prime}}} \chi(F / \mathfrak{A})+\sum_{\substack{\mathfrak{A} \geq 0 \\
\operatorname{deg}(\mathfrak{A})=n \\
\mathfrak{A}+\mathfrak{B} \notin 2 \operatorname{Div}(K)}} \chi(F / \mathfrak{A}) \\
& =\chi\left(F / \mathfrak{B}^{\prime}\right) \quad \sum_{\substack{\mathfrak{C}^{\prime} \geq 0 \\
2 \operatorname{deg}\left(\mathfrak{C}^{\prime}\right)=n-\operatorname{deg}\left(\mathfrak{B}^{\prime}\right)}} \chi\left(F / 2 \mathfrak{C}^{\prime}\right)+\sum_{\substack{\mathfrak{A} \geq 0 \\
\operatorname{deg}(\mathfrak{A})=n \\
\mathfrak{A}+\mathfrak{B} \notin 2 \operatorname{Div}(K)}} \chi(F / \mathfrak{A}),
\end{aligned}
$$


so that by (0)

$$
\begin{aligned}
\left|\sum_{\substack{\mathfrak{A} \geq 0 \\
\operatorname{deg}(\mathfrak{A})=n \\
\mathfrak{A}+\mathfrak{B} \notin 2 \operatorname{Div}(K)}} \chi(F / \mathfrak{A})\right| & =\left|\chi\left(F / \mathfrak{B}^{\prime}\right) \sum_{\substack{\mathfrak{C}^{\prime} \geq 0 \\
2 \operatorname{deg}\left(\mathfrak{C}^{\prime}\right)=n-\operatorname{deg}\left(\mathfrak{B}^{\prime}\right)}} \chi\left(F / 2 \mathfrak{C}^{\prime}\right)\right| \\
& \leq \sum_{\substack{\mathfrak{C}^{\prime} \geq 0 \\
2 \operatorname{deg}\left(\mathfrak{C}^{\prime}\right)=n-\operatorname{deg}\left(\mathfrak{B}^{\prime}\right)}} 1 \\
& \ll q^{\left(n-\operatorname{deg}\left(\mathfrak{B}^{\prime}\right)\right) / 2},
\end{aligned}
$$

where the implicit constant depends on $K$ only. Using this, we see that

$$
\left|\sum_{\substack{\mathfrak{A} \geq 0 \\ \operatorname{deg}(\mathfrak{A})=n \\ \mathfrak{A}+\mathfrak{B} \notin 2 \operatorname{Div}(K)}} \chi(F / \mathfrak{A}) q^{-s \operatorname{deg}(\mathfrak{A})}\right| \ll q^{-\operatorname{deg}\left(\mathfrak{B}^{\prime}\right) / 2} q^{-n(\Re(s)-1 / 2)} .
$$

Since $\Re(s), \Re(t)>1 / 2+\epsilon$,

$$
\sum_{n>2 m-2 g_{K}} q^{-n(\Re(s)-1 / 2)} \ll q^{-2 m(\Re(s)-1 / 2)}
$$

and

$$
\begin{aligned}
\sum_{\mathfrak{B} \geq 0} q^{-\Re(t) \operatorname{deg}(\mathfrak{B})} q^{-\operatorname{deg}\left(\mathfrak{B}^{\prime}\right) / 2} & =\sum_{\mathfrak{B} \geq 0} q^{-(\Re(t)+1 / 2) \operatorname{deg}\left(\mathfrak{B}^{\prime}\right)} q^{-2 \Re(t) \operatorname{deg}\left(\mathfrak{B}^{\prime \prime}\right)} \\
& \leq \sum_{\mathfrak{B}^{\prime} \geq 0} q^{-(\Re(t)+1 / 2) \operatorname{deg}\left(\mathfrak{B}^{\prime}\right)} \sum_{\mathfrak{B}^{\prime \prime} \geq 0} q^{-2 \Re(t) \operatorname{deg}\left(\mathfrak{B}^{\prime \prime}\right)} \\
& \ll 1
\end{aligned}
$$

The lemma follows from (34)-(36).

Proof of Theorem 1. By Proposition 7 (specifically, the estimate for the number of quadratic extensions of genus $m$ ), Lemma 25 and the multiplicative nature of $\chi(F / *)$,

$$
\begin{aligned}
& \sum_{\substack{[F: K]=2 \\
g_{F}=m, q_{F}=q}} \sum_{\substack{\mathfrak{A}, \mathfrak{B} \geq 0 \\
\mathfrak{A}+\mathfrak{B} \notin 2 \operatorname{Div}(K) \\
\operatorname{deg}(\mathfrak{A})>2 m-2 g_{K} \text { or } \operatorname{deg}(\mathfrak{B})>2 m-2 g_{K}}} q^{-s \operatorname{deg}(\mathfrak{A})} q^{-t \operatorname{deg}(\mathfrak{B})} \chi(F /(\mathfrak{A}+\mathfrak{B})) \mid \\
& \ll q^{2 m(3 / 2-\Re(s))}+q^{2 m(3 / 2-\Re(t))} .
\end{aligned}
$$


Suppose that $q$ is odd. By Proposition 5 with $\mathfrak{A}+\mathfrak{B}$ in place of $\mathfrak{C}$,

$$
\left|\sum_{\substack{[F: K]=2 \\ g F=m, q_{F}=q}} \chi(F /(\mathfrak{A}+\mathfrak{B}))\right| \ll q^{m+(\epsilon+1 / 4) \operatorname{deg}(\mathfrak{A}+\mathfrak{B})} .
$$

This in conjunction with (0) yields

$$
\begin{array}{|l}
\sum_{\substack{\mathfrak{A}, \mathfrak{B} \geq 0 \\
\mathfrak{A}+2 \operatorname{Div}(K) \\
\operatorname{deg}(\mathfrak{A}), \operatorname{deg}(\mathfrak{B}) \leq 2 m-2 g_{K}}} q^{-s \operatorname{deg}(\mathfrak{A})} q^{-t \operatorname{deg}(\mathfrak{B})} \sum_{\substack{[F: K]=2 \\
g_{F}=m, q_{F}=q}} \chi(F /(\mathfrak{A}+\mathfrak{B})) \mid \\
\ll q^{m} \sum_{\substack{\mathfrak{A} \geq 0 \\
\operatorname{deg}(\mathfrak{A}) \leq 2 m-2 g_{K}}} q^{(\epsilon+1 / 4-\Re(s)) \operatorname{deg}(\mathfrak{A})} \sum_{\substack{\mathfrak{B} \geq 0 \\
\operatorname{deg}(\mathfrak{B}) \leq 2 m-2 g_{K}}} q^{(\epsilon+1 / 4-\Re(t)) \operatorname{deg}(\mathfrak{B})} \\
\ll q^{m}\left(1+q^{2 m(5 / 4+\epsilon-\Re(s))}\right)\left(1+q^{2 m(5 / 4+\epsilon-\Re(t))}\right) .
\end{array}
$$

Suppose that $q$ is even. By Proposition 6 with $\mathfrak{A}+\mathfrak{B}$ in place of $\mathfrak{C}$,

$$
\left|\sum_{\substack{[F: K]=2 \\ g F=m, q_{F}=q}} \chi(F /(\mathfrak{A}+\mathfrak{B}))\right| \ll q^{m+\epsilon \operatorname{deg}(\mathfrak{A}+\mathfrak{B})} .
$$

This in conjunction with (0) yields

$$
\begin{aligned}
& \left|\sum_{\substack{\mathfrak{A}, \mathfrak{B} \geq 0 \\
\mathfrak{A}+\mathfrak{B} \notin 2 \operatorname{Div}(K) \\
\operatorname{deg}(\mathfrak{A}), \operatorname{deg}(\mathfrak{B}) \leq 2 m-2 g_{K}}} q^{-s \operatorname{deg}(\mathfrak{A})} q^{-t \operatorname{deg}(\mathfrak{B})} \sum_{\substack{[F: K]=2 \\
g_{F}=m, q_{F}=q}} \chi(F /(\mathfrak{A}+\mathfrak{B}))\right| \\
& \ll q^{m} \sum_{\substack{\mathfrak{A} \geq 0 \\
\operatorname{deg}(\mathfrak{A}) \leq 2 m-2 g_{K}}} q^{(\epsilon-\Re(s)) \operatorname{deg}(\mathfrak{A})} \sum_{\substack{\mathfrak{B} \geq 0 \\
\operatorname{deg}(\mathfrak{B}) \leq 2 m-2 g_{K}}} q^{(\epsilon-\Re(t)) \operatorname{deg}(\mathfrak{B})} \\
& \ll q^{m}\left(1+q^{2 m(1+\epsilon-\Re(s))}\right)\left(1+q^{2 m(1+\epsilon-\Re(t))}\right) .
\end{aligned}
$$

Theorem 1 follows from Lemma 24 and (37)-(39).

Proof of Theorem 2. Since we have a $\mu(\mathfrak{B})$ factor now, instead of (37) we have

$$
\sum_{\substack{[F: K]=2 \\ g_{F}=m, q_{F}=q}} \sum_{\substack{\mathfrak{A}, \mathfrak{B} \geq 0 \\ \mathfrak{A}+\mathfrak{B} \notin 2 \operatorname{Div}(K) \\ \operatorname{deg}(\mathfrak{A})>2 m-2 g_{K}}} \mu(\mathfrak{B}) q^{-s \operatorname{deg}(\mathfrak{A})} q^{-t \operatorname{deg}(\mathfrak{B})} \chi(F /(\mathfrak{A}+\mathfrak{B})) \mid
$$


Suppose $q$ is odd. Using Proposition 5 with $\mathfrak{A}+\mathfrak{B}$ in place of $\mathfrak{C}$ we have

$$
\begin{aligned}
& \left|\sum_{\substack{\mathfrak{A}, \mathfrak{B} \geq 0 \\
\mathfrak{A}+\mathfrak{B} \notin 2 \operatorname{Div}(K) \\
\operatorname{deg}(\mathfrak{A})+\operatorname{deg}(\mathfrak{B}) \leq 4 m \\
\operatorname{deg}(\mathfrak{A}) \leq 2 m-2 g_{K}}} \mu(\mathfrak{B}) q^{-s \operatorname{deg}(\mathfrak{A})} q^{-t \operatorname{deg}(\mathfrak{B})} \sum_{\substack{[F: K]=2 \\
g_{F}=m, q_{F}=q}} \chi(F /(\mathfrak{A}+\mathfrak{B}))\right| \\
& \ll q^{m} \sum_{\substack{\mathfrak{A} \geq 0 \\
\operatorname{deg}(\mathfrak{A}) \leq 2 m-2 g_{K}}} q^{(\epsilon+1 / 4-\Re(s)) \operatorname{deg}(\mathfrak{A})} \sum_{\substack{\mathfrak{B} \geq 0 \\
\operatorname{deg}(\mathfrak{B}) \leq 4 m-\operatorname{deg}(\mathfrak{A})}} q^{(\epsilon+1 / 4-\Re(t)) \operatorname{deg}(\mathfrak{B})} \\
& \ll q^{m} \quad \sum_{\mathfrak{A} \geq 0} \quad q^{(\epsilon+1 / 4-\Re(s)) \operatorname{deg}(\mathfrak{A})}\left(1+q^{(5 / 4+\epsilon-\Re(t))(4 m-\operatorname{deg}(\mathfrak{A}))}\right) \\
& \operatorname{deg}(\mathfrak{A}) \leq 2 \mathrm{z} m-2 g_{K} \\
& \ll q^{m}\left(1+q^{2 m(5 / 4+\epsilon-\Re(s))}+q^{2 m(5 / 2+2 \epsilon-2 \Re(t))}+q^{2 m(5 / 2+2 \epsilon-\Re(s)-\Re(t))}\right),
\end{aligned}
$$

and similarly, since $\Re(t)>1+\epsilon$

$$
\begin{aligned}
& \sum_{\substack{\mathfrak{A}, \mathfrak{B} \geq 0 \\
\mathfrak{A}+\mathfrak{B} \notin 2 \operatorname{Div}(K) \\
\operatorname{eg}(\mathfrak{A})+\operatorname{deg}(\mathfrak{B})>4 m \\
\operatorname{deg}(\mathfrak{A}) \leq 2 m-2 g_{K}}} \mu(\mathfrak{B}) q^{-s \operatorname{deg}(\mathfrak{A})} q^{-t \operatorname{deg}(\mathfrak{B})} \sum_{\substack{[F: K]=2 \\
g_{F}=m, q_{F}=q}} \chi(F /(\mathfrak{A}+\mathfrak{B})) \\
& \ll q^{2 m} \sum_{\substack{\mathfrak{A} \geq 0 \\
\operatorname{deg}(\mathfrak{H}) \leq 2 m-2 g_{K}}} q^{-\Re(s) \operatorname{deg}(\mathfrak{A})} \quad \sum_{\substack{\mathfrak{B} \geq 0\\
}} q^{-\Re(t) \operatorname{deg}(\mathfrak{B})} \\
& \operatorname{deg}(\mathfrak{A}) \leq \overline{2} m-2 g_{K} \quad \operatorname{deg}(\mathfrak{B})>4 m-\operatorname{deg}(\mathfrak{A}) \\
& \ll q^{2 m} q^{-4 m(\Re(t)-1)} \sum_{\substack{\mathfrak{A} \geq 0 \\
\operatorname{deg}(\mathfrak{A}) \leq \overline{2} m-2 g_{K}}} q^{(\Re(t)-1-\Re(s)) \operatorname{deg}(\mathfrak{A})} \\
& \ll q^{m}\left(q^{2 m(5 / 2+2 \epsilon-2 \Re(t))}+q^{2 m(5 / 2-\Re(s)-\Re(t))}\right) .
\end{aligned}
$$

Suppose that $q$ is even. By Proposition 6 with $\mathfrak{A}+\mathfrak{B}$ in place of $\mathfrak{C}$ and $\epsilon / 2$ in place of $\epsilon$, and using $\Re(t)>1+\epsilon$ we get

$$
\begin{aligned}
& \sum_{\substack{\mathfrak{A}, \mathfrak{B} \geq 0 \\
\mathfrak{A}+\mathfrak{B} \notin 2 \operatorname{Div}(K) \\
\operatorname{deg}(\mathfrak{A}) \leq 2 m-2 g_{K}}} \mu(\mathfrak{B}) q^{-s \operatorname{deg}(\mathfrak{A})} q^{-t \operatorname{deg}(\mathfrak{B})} \sum_{\substack{[F: K]=2 \\
g_{F}=m, q_{F}=q}} \chi(F /(\mathfrak{A}+\mathfrak{B})) \\
& \ll q^{m} \sum_{\substack{\mathfrak{A} \geq 0 \\
\operatorname{deg}(\mathfrak{A}) \leq \overline{2} m-2 g_{K}}} q^{(\epsilon-\Re(s)) \operatorname{deg}(\mathfrak{A})} \sum_{\mathfrak{B} \geq 0} q^{(\epsilon-\Re(t)) \operatorname{deg}(\mathfrak{B})} \\
& \ll q^{m}\left(1+q^{2 m(1+\epsilon-\Re(s))}\right) .
\end{aligned}
$$


Theorem 2 follows from Lemma 24 and (40)-(43).

Proof of Theorem 3. Since we now have both a $\mu(\mathfrak{A})$ and $\mu(\mathfrak{B})$ term, we are unable to utilize Lemma 25.

Suppose $q$ is odd. Then by Proposition 5 we have

$$
\begin{aligned}
& \left|\sum_{\substack{\mathfrak{A}, \mathfrak{B} \geq 0 \\
\mathfrak{A}+\mathfrak{B} \notin 2 \operatorname{Div}(K) \\
\operatorname{deg}(\mathfrak{A})+\operatorname{deg}(\mathfrak{B}) \leq 4 m}} \mu(\mathfrak{A}) \mu(\mathfrak{B}) q^{-s \operatorname{deg}(\mathfrak{A})} q^{-t \operatorname{deg}(\mathfrak{B})} \sum_{\substack{[F: K]=2 \\
g F \\
=}} \chi(F /(\mathfrak{A}+\mathfrak{B}))\right| \\
& \ll q^{m} \sum_{\substack{\mathfrak{A} \geq 0 \\
\operatorname{deg}(\mathfrak{A}) \leq 4 m}} q^{(\epsilon+1 / 4-\Re(s)) \operatorname{deg}(\mathfrak{A})} \sum_{\substack{\mathfrak{B} \geq 0 \\
\operatorname{deg}(\mathfrak{B}) \leq 4 m-\operatorname{deg}(\mathfrak{A})}} q^{(\epsilon+1 / 4-\Re(t)) \operatorname{deg}(\mathfrak{B})} \\
& \ll q^{m} \sum_{\substack{\mathfrak{A} \geq 0 \\
\operatorname{deg}(\mathfrak{A}) \leq 4 m}} q^{(\epsilon / 2+1 / 4-\Re(s)) \operatorname{deg}(\mathfrak{A})}\left(1+q^{(4 m-\operatorname{deg}(\mathfrak{A})(5 / 4+\epsilon-\Re(t))}\right) \\
& \ll q^{m}\left(1+q^{2 m(5 / 2+\epsilon-2 \Re(s))}+q^{2 m(5 / 2+\epsilon-2 \Re(t))}\right) .
\end{aligned}
$$

Similarly, we get

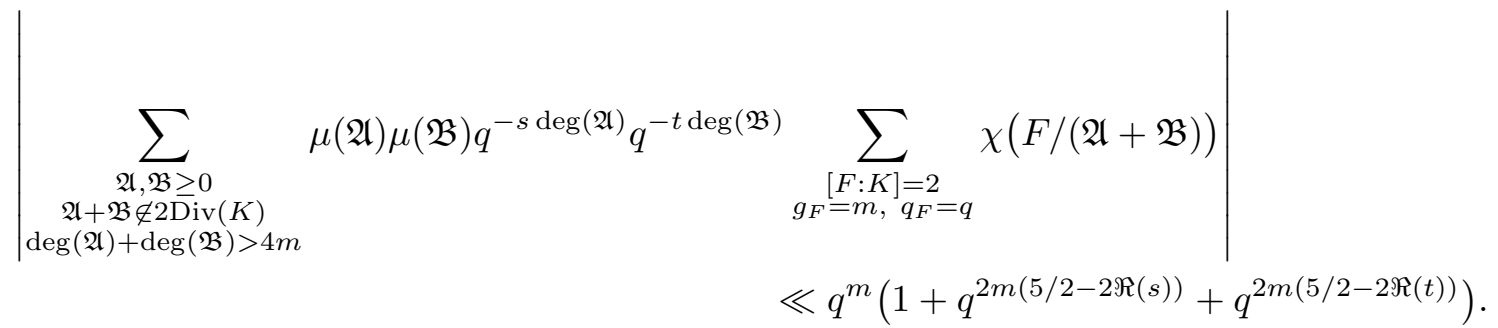

Suppose $q$ is even. Then by Proposition 6 with $\epsilon$ replaced by $\epsilon / 2$ and using $\Re(s), \Re(t)>1+\epsilon$, we have

$$
\begin{aligned}
& \left|\sum_{\substack{\mathfrak{A}, \mathfrak{B} \geq 0 \\
\mathfrak{A}+\mathfrak{B} \notin 2 \operatorname{Div}(K)}} \mu(\mathfrak{A}) \mu(\mathfrak{B}) q^{-s \operatorname{deg}(\mathfrak{A})} q^{-t \operatorname{deg}(\mathfrak{B})} \sum_{\substack{[F: K]=2 \\
g_{F}=m, q_{F}=q}} \chi(F /(\mathfrak{A}+\mathfrak{B}))\right| \\
& \ll q^{m} \sum_{\mathfrak{A} \geq 0} q^{(\epsilon / 2-\Re(s)) \operatorname{deg}(\mathfrak{A})} \sum_{\mathfrak{B} \geq 0} q^{(\epsilon / 2-\Re(t)) \operatorname{deg}(\mathfrak{B})} \\
& \ll q^{m} \text {. }
\end{aligned}
$$

Theorem 3 follows from Lemma 24 and (44)-(46). 


\section{REFERENCES}

[1] G. Chinta, S. Friedberg and J. Hoffstein, Multiple Dirichlet series and automorphic forms, Proc. Sympos. Pure Math. 75 (2006), 3-41.

[2] J. Ellenberg and A. Venkatesh, Counting extensions of function fields with bounded discriminant and specified Galois group, Prog. Math. 235 (2005), 151-168.

[3] B. Fisher and S. Friedberg, Double Dirichlet series over function fields, Compos. Math. 140 (2004), 613-630.

[4] D. Goldfeld and J. Hoffstein, Eisenstein series of $\frac{1}{2}$-integral weight and the mean value of real Dirichlet $L$-series, Invent. Math. 80 (1985), 185-208.

[5] J. Hoffstein and M. Rosen, Average values of L-series in function fields, J. Reine Angew. Math. 426 (1992), 117-150.

[6] D. Kettlestrings and J.L. Thunder, The number of function fields with given genus, Contem. Math. 587 (2013), 141-149.

[7] - Counting points of given height that generate a quadratic extension of a function field, preprint (2013).

[8] M. Rosen, Number Theory in Function Fields, Springer-Verlag, New York, 2002.

[9] W.M. Schmidt, Northcott's theorem on heights II. The quadratic case, Acta Arith. 70 (1995), 343-375.

[10] C.L. Siegel, The average measure of quadratic forms with given discriminant and signature, Annals of Math. 45 (1944), 667-685.

[11] H. Stichtenoth, Algebraic Function Fields and Codes, Springer-Verlag, Berlin, 1993.

[12] J.L. Thunder and M. Widmer, Counting points of fixed degree and given height over function fields, Bull. London Math. Soc. 45 (2) (2013), 283-300.

[13] A. Weil, Basic Number Theory, Springer-Verlag, Berlin, 1974.

Mathematical Sciences Dept., Northern Illinois Univ., DeKalb, IL 60115

E-mail address: jthunder@ math.niu.edu 